\title{
Structural Alternatives for the Formation of
}

\section{Chlorophosphine-Phosphenium Complexes:}

\section{Supporting Information}

\author{
Sebastian Burck, ${ }^{a}$ Dietrich Gudat, *
}

Contribution from the

Institut für Anorganische Chemie, Universität Stuttgart, Pfaffenwaldring 55, 70550

${ }^{a}$ current address:

Section Organic and Inorganic Chemistry, Vrije Universiteit Amsterdam, De Boelelaan 1083, 1081 HV Amsterdam, Netherlands

E-mail: gudat@iac.uni-stuttgart.de

Contents: Complete ref 25; extended computational details, including geometric and energy data for gas phase structures of the cations $\mathbf{1 b}-\mathbf{3 b}$, halophosphines $\mathbf{1 b}[\mathrm{X}](\mathrm{X}=\mathrm{F}, \mathrm{Cl}, \mathrm{Br}), \mathbf{2 b}[\mathrm{Cl}], \mathbf{3 b}[\mathrm{Cl}]$, and cation-halophosphine complexes $\mathbf{4 b}[\mathrm{X}](\mathrm{X}=\mathrm{F}, \mathrm{Cl}, \mathrm{Br}), \mathbf{4}^{\prime} \mathbf{b}[\mathrm{F}], \mathbf{5 b}[\mathrm{Cl}], \mathbf{6} \mathbf{b}[\mathrm{Cl}], \mathbf{6} ' \mathbf{b}[\mathrm{Cl}]$, and solution structures for $\mathbf{1 b}-\mathbf{3 b}, \mathbf{1 b}[\mathrm{X}](\mathrm{X}=\mathrm{F}, \mathrm{Cl}, \mathrm{Br}), \mathbf{2 b}[\mathrm{Cl}], \mathbf{3 b}[\mathrm{Cl}], \mathbf{4 b}[\mathrm{X}](\mathrm{X}=\mathrm{F}, \mathrm{Cl}), \mathbf{4} ' \mathrm{~b}[\mathrm{~F}], \mathbf{5 b}[\mathrm{Cl}], \mathbf{6 b}[\mathrm{Cl}]$, $\mathbf{6} ' \mathbf{b}[\mathrm{Cl}]\left(\right.$ in $\left.\mathrm{CH}_{2} \mathrm{Cl}_{2}\right)$ and $\mathbf{1 b}[\mathrm{Cl}]\left(\right.$ in $\left.\mathrm{CH}_{3} \mathrm{CN}\right)$. 
Reference 25: Gaussian 03, Revision D.02, M. J. Frisch, G. W. Trucks, H. B. Schlegel, G. E. Scuseria, M. A. Robb, J. R. Cheeseman, J. A. Montgomery, Jr., T. Vreven, K. N. Kudin, J. C. Burant, J. M. Millam, S. S. Iyengar, J. Tomasi, V. Barone, B. Mennucci, M. Cossi, G. Scalmani, N. Rega, G. A. Petersson, H. Nakatsuji, M. Hada, M. Ehara, K. Toyota, R. Fukuda, J. Hasegawa, M. Ishida, T. Nakajima, Y. Honda, O. Kitao, H. Nakai, M. Klene, X. Li, J. E. Knox, H. P. Hratchian, J. B. Cross, V. Bakken, C. Adamo, J. Jaramillo, R. Gomperts, R. E. Stratmann, O. Yazyev, A. J. Austin, R. Cammi, C. Pomelli, J. W. Ochterski, P. Y. Ayala, K. Morokuma, G. A. Voth, P. Salvador, J. J. Dannenberg, V. G. Zakrzewski, S. Dapprich, A. D. Daniels, M. C. Strain, O. Farkas, D. K. Malick, A. D. Rabuck, K. Raghavachari, J. B. Foresman, J. V. Ortiz, Q. Cui, A. G. Baboul, S. Clifford, J. Cioslowski, B. B. Stefanov, G. Liu, A. Liashenko, P. Piskorz, I. Komaromi, R. L. Martin, D. J. Fox, T. Keith, M. A. AlLaham, C. Y. Peng, A. Nanayakkara, M. Challacombe, P. M. W. Gill, B. Johnson, W. Chen, M. W. Wong, C. Gonzalez, and J. A. Pople, Gaussian, Inc., Wallingford CT, 2004.

General computational details: All calculations of gas phase structures were carried out by performing first a complete energy optimization of all geometric parameters (input keywords “\# b3lyp/6-31+g** int(ultrafine) opt") using point group symmetries given in Table 2 . After the stationary points had been located, in each case a frequency calculation (input keywords "\# b31yp/6-31+g** int(ultrafine) freq(noraman) pop(nbo)") was performed. The given relative energies were determined by calculating the difference in absolute energies between complexes and the appropriate isolated fragments, resp., and converting the result into $\mathrm{kcal} \mathrm{mol}^{-1}$. The molecular structures of key compounds were re-optimized at the mpw1k/6-31+g** (input keywords \# MPWpw91/6-31+g** pop(none) $\operatorname{int}(u l t r a f i n e) \operatorname{iop}(5 / 45=10000428) \operatorname{iop}(5 / 46=05720572) \operatorname{iop}(5 / 47=10001000)$ opt $)$ and $\mathrm{mp} 2 / / 6-31+\mathrm{g}^{* *}$ (input keywords mp2/6-31+g** pop(none) opt) levels, respectively. Energies and molecular geometries of species in solution were determined after energy re-optimization of the b3lyp-structures (input keywords “\# b3lyp/6-31+g** int(ultrafine) opt(readfc) guess(check) geom(check) scrf(cosmo, solvent $=\mathrm{XXXX}) "(\mathrm{XXXX}=\mathrm{CH} 2 \mathrm{Cl} 2$ or $\mathrm{CH} 3 \mathrm{CN})$ which were performed using the gas phase structures as starting points. In several cases the optimizations had to be restarted with minor modifications in 
atomic coordinates, due to numerical instabilities in the program code or problems in the construction of the solvent cavity. In the case of $\mathbf{3 b}, \mathbf{4 b}[\mathrm{F}], \mathbf{4}^{\prime} \mathbf{b}[\mathrm{F}], \mathbf{4 b}[\mathrm{Cl}], \mathbf{5 b}[\mathrm{Cl}], \mathbf{6 b}[\mathrm{Cl}]$ the optimization runs had to be started with the keyword "opt(loose)" to force less tight cutoff criteria in order to overcome convergence problems which originated apparently also in problems related to the construction of solvent cavities and caused the optimization run to oscillate between two nearly identical molecular structures rather than to converge.

Computational results / final atomic coordinates and energies for gas phase structures at b3lyp/6-31+g** level:

\section{1,3-Dimethyl-1,3,2-diazaphospholenium cation 1b:}

\begin{tabular}{|c|c|c|c|c|c|}
\hline \multirow{2}{*}{$\begin{array}{l}\text { Center } \\
\text { Number }\end{array}$} & \multirow{2}{*}{$\begin{array}{l}\text { Atomic } \\
\text { Number }\end{array}$} & \multirow{2}{*}{$\begin{array}{l}\text { Atomic } \\
\text { Type }\end{array}$} & \multicolumn{3}{|c|}{ Coordinates (Angstroms) } \\
\hline & & & $\mathrm{X}$ & $\mathrm{Y}$ & Z \\
\hline & & & & & \\
\hline 1 & 15 & $\odot$ & $\odot .126148$ & $-\odot .109117$ & -1.13011 \\
\hline 2 & 7 & $\odot$ & 0.143878 & 1.182496 & -0.03228 \\
\hline 3 & 6 & $\odot$ & -0.060664 & 0.806121 & 1.26575 \\
\hline 4 & 6 & $\odot$ & -0.234551 & -0.550891 & 1.37736 \\
\hline 5 & 7 & $\odot$ & -0.158253 & -1.170056 & 0.16114 \\
\hline 6 & 6 & $\odot$ & -0.312656 & -2.633585 & 0.00749 \\
\hline 7 & 6 & $\odot$ & 0.359057 & 2.593523 & -0.42222 \\
\hline 8 & 1 & $\odot$ & -0.072397 & 1.536972 & 2.06336 \\
\hline 9 & 1 & $\odot$ & -0.412668 & -1.117423 & 2.28168 \\
\hline 10 & 1 & $\odot$ & 0.498257 & 2.653851 & -1.50213 \\
\hline 11 & 1 & $\odot$ & -0.513991 & 3.184618 & -0.13888 \\
\hline 12 & 1 & $\odot$ & 1.251846 & 2.975052 & 0.07703 \\
\hline 13 & 1 & $\odot$ & $-\odot .213873$ & -2.899826 & -1.04539 \\
\hline 14 & 1 & $\odot$ & $\odot .464849$ & -3.141850 & 0.58111 \\
\hline 15 & 1 & $\odot$ & -1.300729 & -2.932722 & 0.36264 \\
\hline
\end{tabular}

\begin{tabular}{|c|c|c|c|}
\hline SCF Done: & $\begin{array}{c}E(R B+H F-L Y P) \\
\text { Convg }= \\
S^{* * 2}=\end{array}$ & $\begin{array}{l}=\quad-607.895554783 \\
0.1448 \mathrm{D}-\odot 8 \\
\odot .0 \odot \odot \odot\end{array}$ & $\begin{array}{l}\text { A.U. after } 1 \text { cycles } \\
-V / T=2.0065\end{array}$ \\
\hline ro-poin & correction= & & ๑.124168 (Hartree/Particl \\
\hline$m$ of ele & ctronic and $z$ & zero-point Ener & -607.771387 \\
\hline um of eles & ctronic and $t$ & thermal Enthalpies= & -607.762947 \\
\hline um of ele & ctronic and $t$ & thermal Free Energies= & -607.803351 \\
\hline
\end{tabular}

\section{1,3-Dimethyl-1,3,2-diazaphospholidinium cation $2 \mathrm{~b}$ :}

\begin{tabular}{|c|c|c|c|c|c|}
\hline Center & Atomic & Atomic & \multicolumn{3}{|c|}{ Coordinates (Angstroms) } \\
\hline Number & Number & Tyре & $\mathrm{X}$ & Y & Z \\
\hline 1 & 6 & 0 & $\odot .764471$ & 1.377889 & 0.10298 \\
\hline 2 & 6 & ○ & -0.764469 & 1.377892 & -0.10298 \\
\hline 3 & 7 & $\odot$ & -1.207524 & -0.033325 & ๑.03118 \\
\hline
\end{tabular}




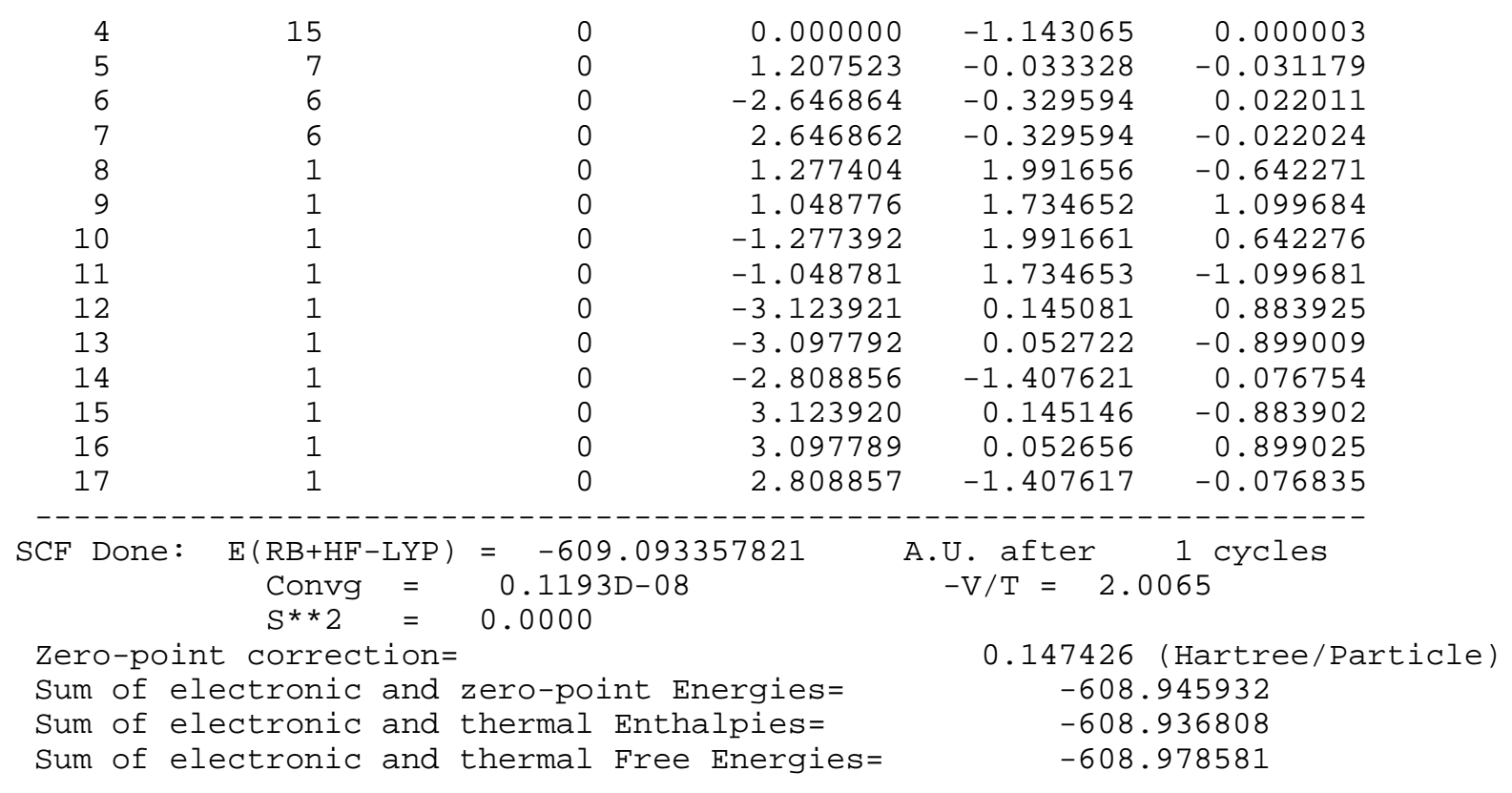

Bis-(Dimethylamino)-phosphenium cation 3b:

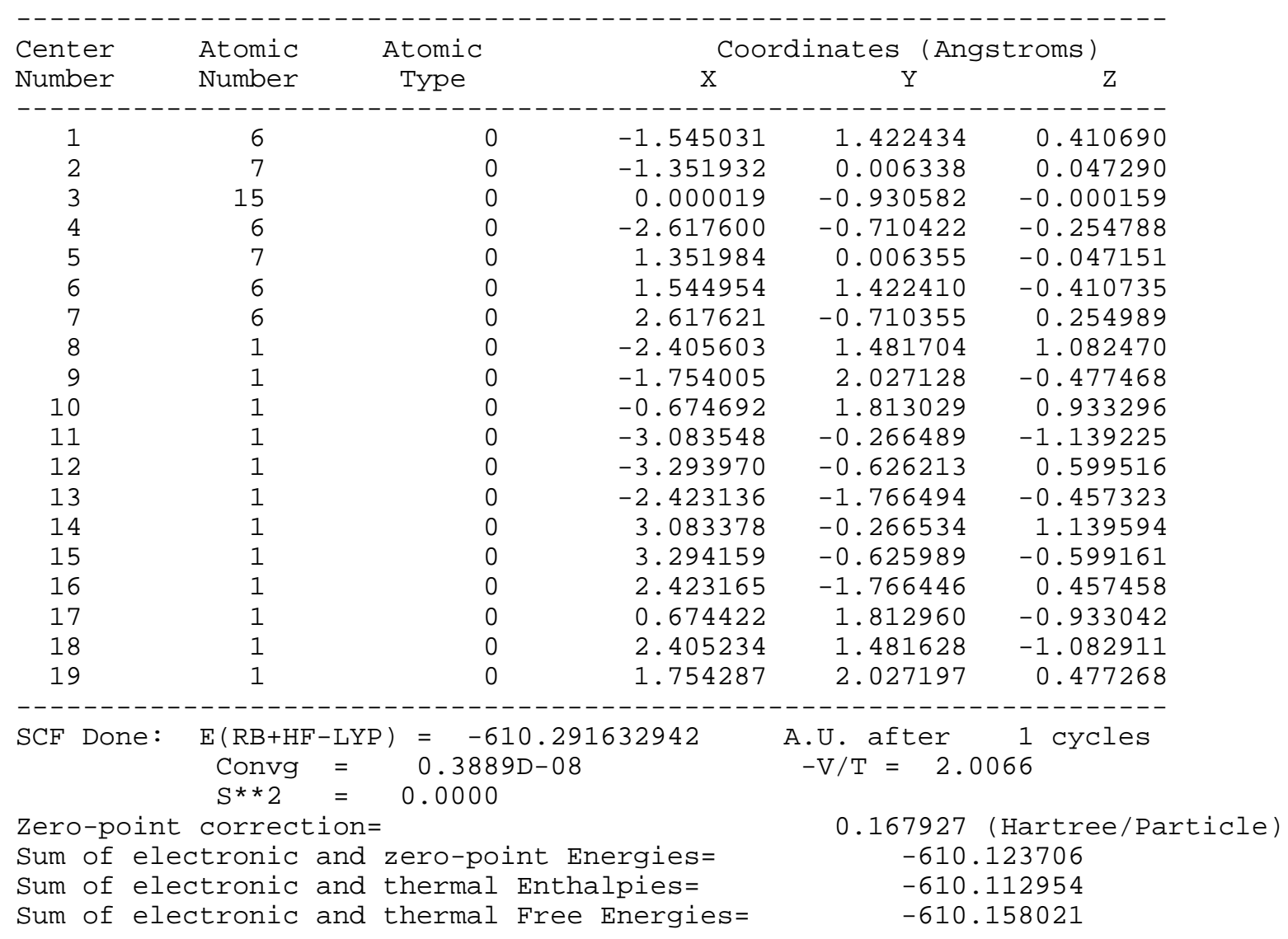

2-Fluoro-1,3-Dimethyl-1,3,2-diazaphospholene 1b[F]: 


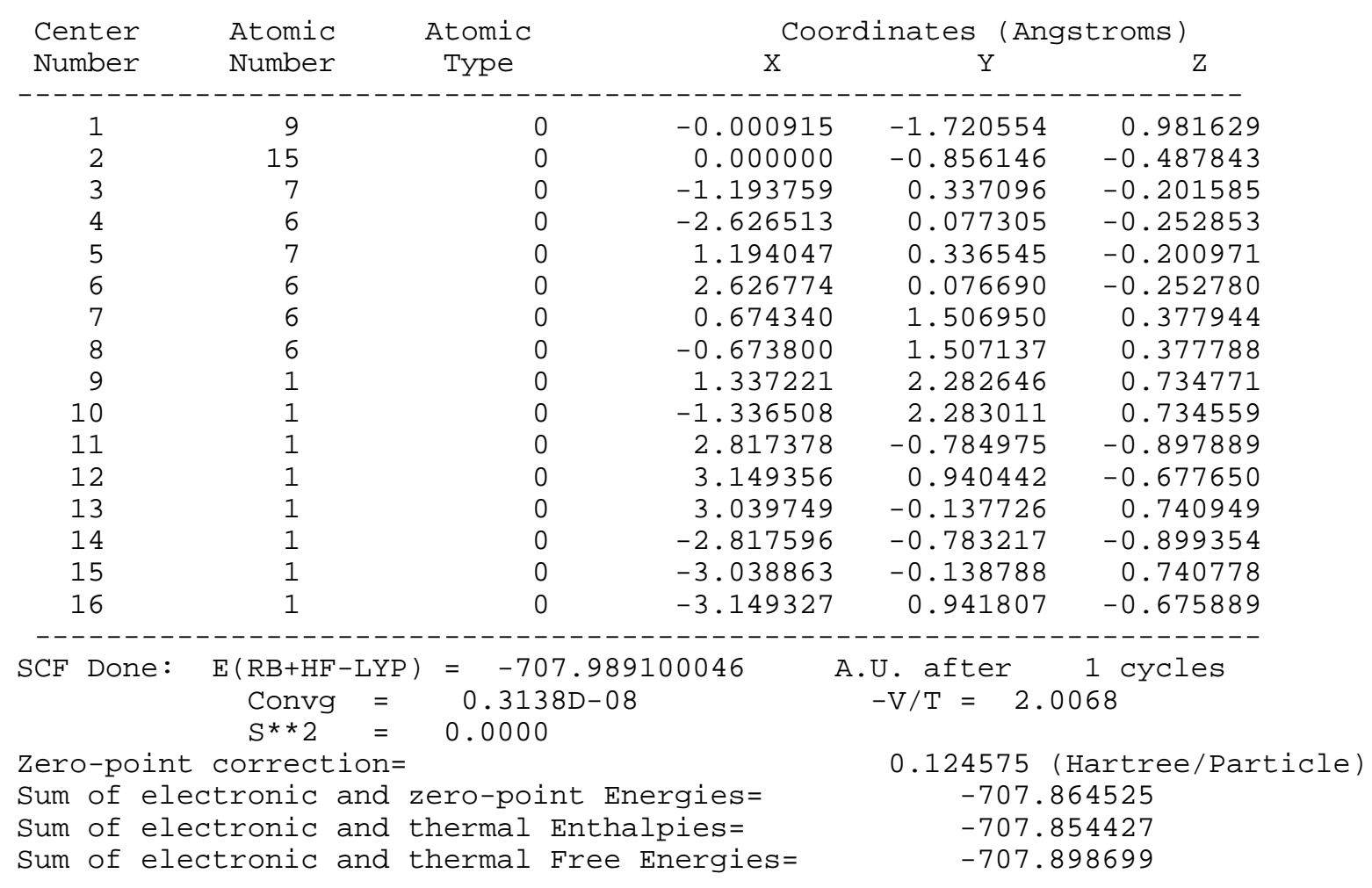

\section{2-Chloro-1,3-Dimethyl-1,3,2-diazaphospholene 1b[Cl]:}

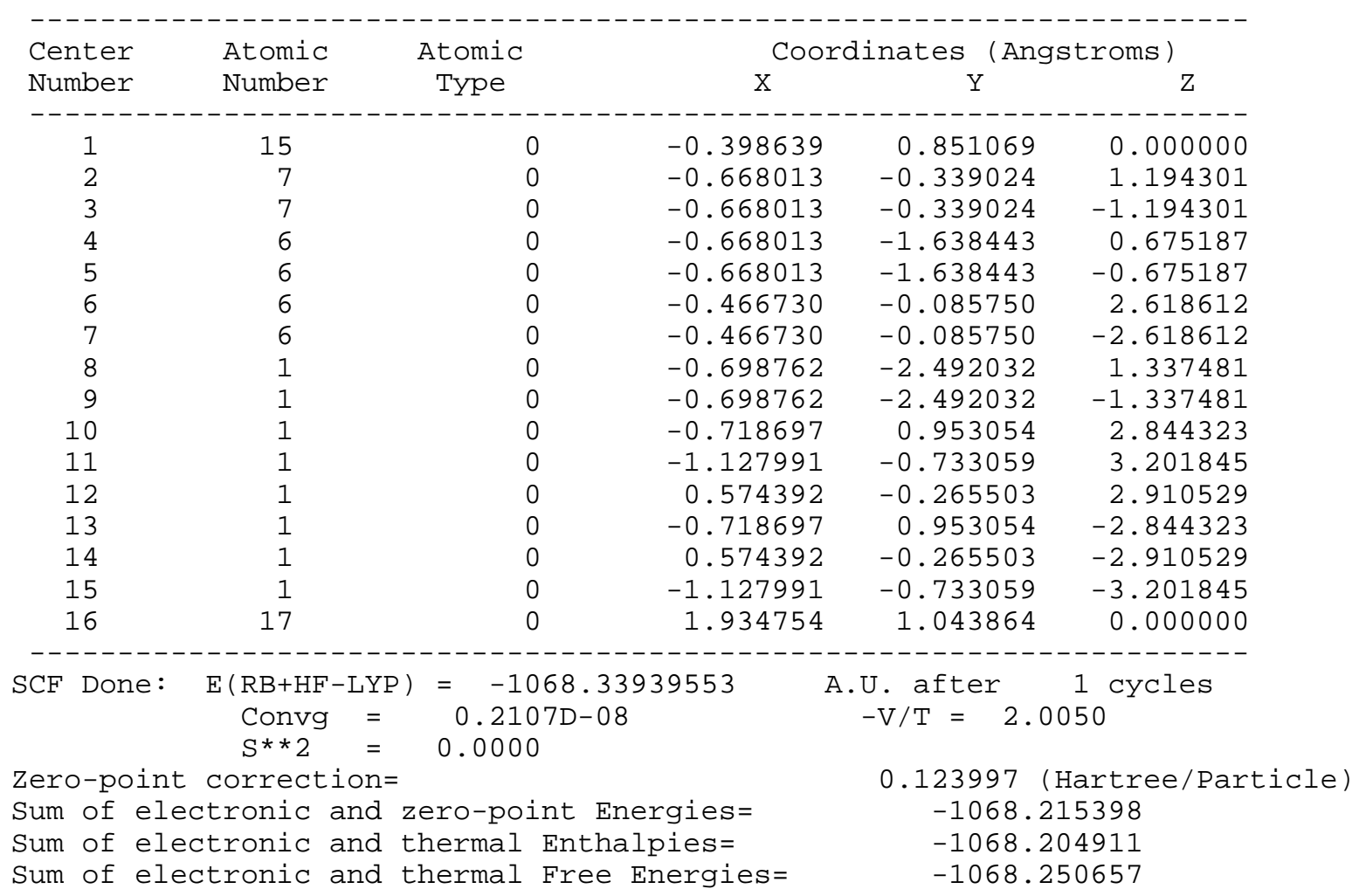

\section{2-Bromo-1,3-Dimethyl-1,3,2-diazaphospholene 1b[Br]:}




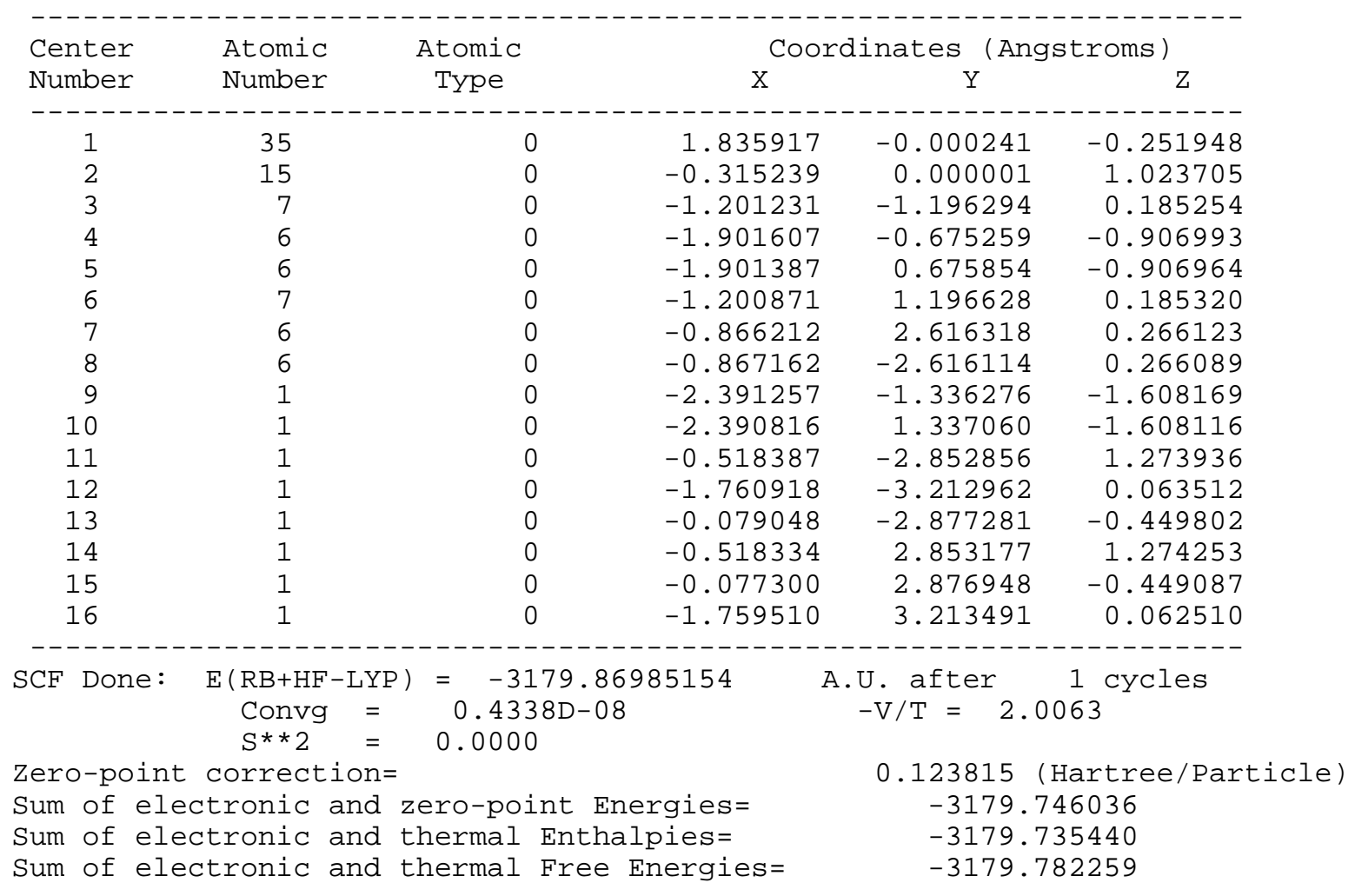

2-Chloro-1,3-Dimethyl-1,3,2-diazaphospholidine 2b[Cl]:

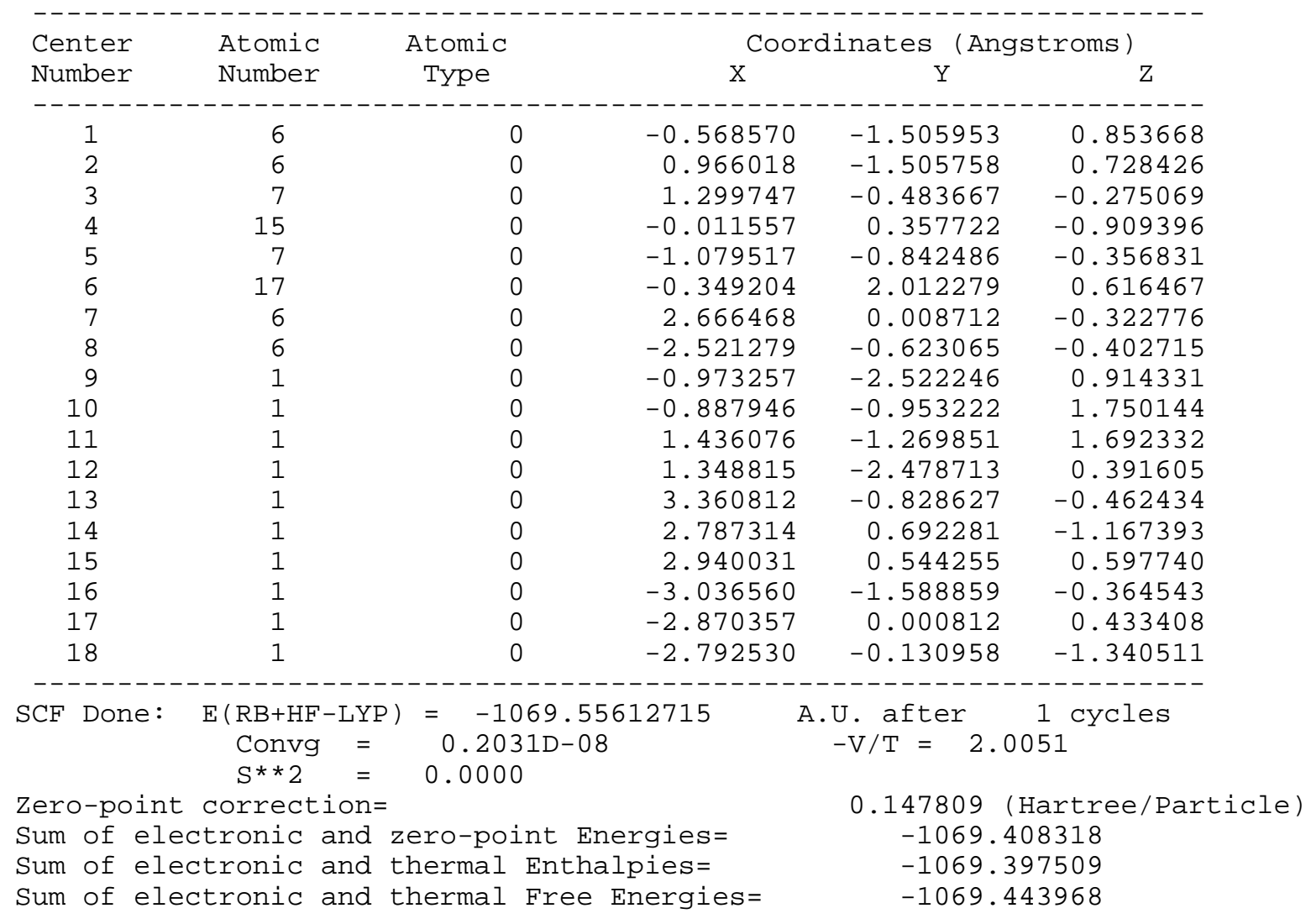


Chloro-bis-(dimethylamino)-phosphine $3 \mathrm{~b}[\mathrm{Cl}]$ :

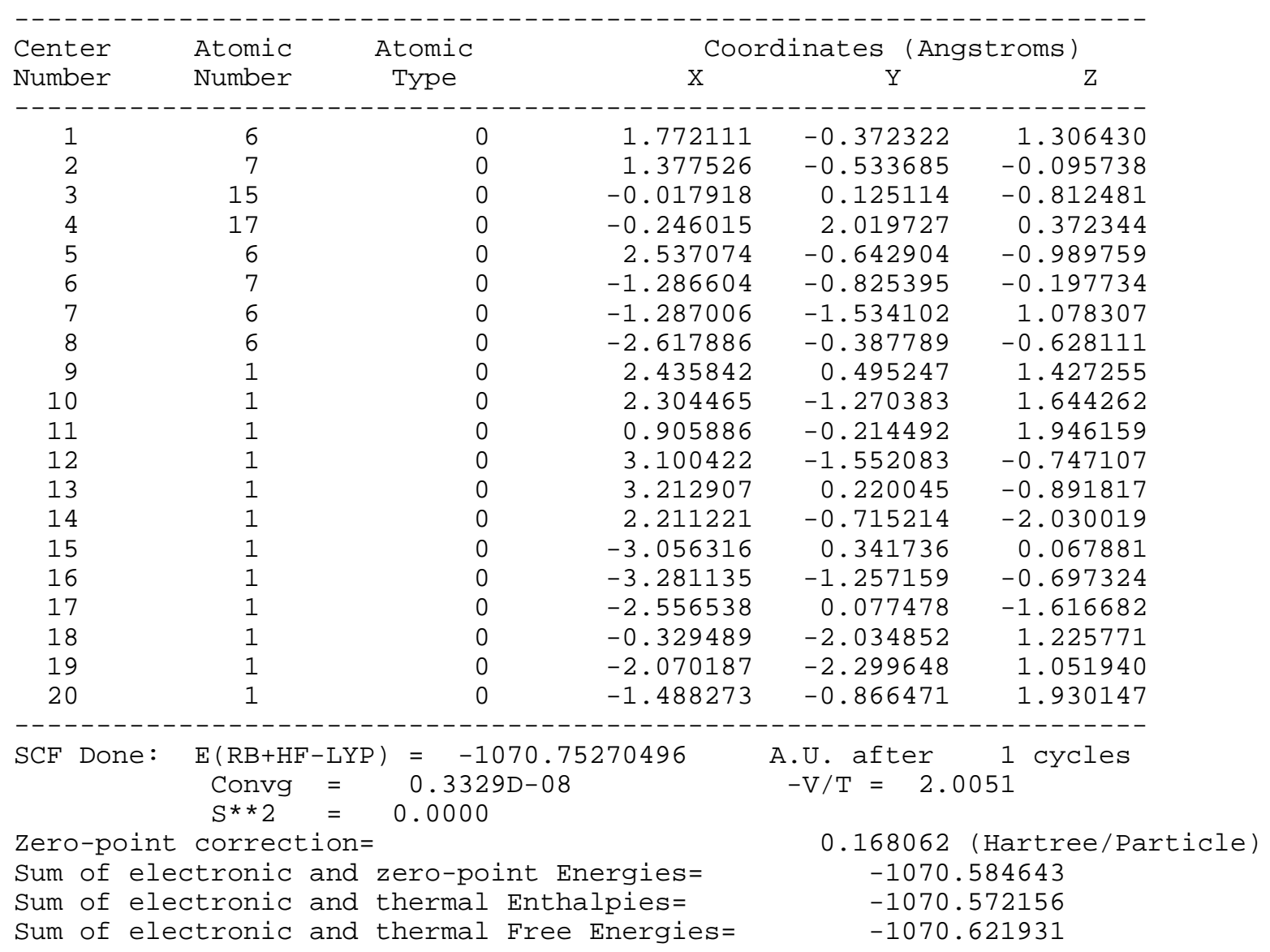

\section{F-bridged 2-Fluoro-1,3-Dimethyl-1,3,2-diazaphospholene / 1,3-Dimethyl-1,3,2-}

\section{diazaphospholenium Complex 4'b[F]:}

\begin{tabular}{|c|c|c|c|c|c|}
\hline \multirow{2}{*}{$\begin{array}{l}\text { Center } \\
\text { Number }\end{array}$} & Atomic & Atomic & \multicolumn{3}{|c|}{ Coordinates (Angstroms) } \\
\hline & Number & Tyре & $X$ & $\mathrm{Y}$ & Z \\
\hline & & & & & $6--------$ \\
\hline 1 & 6 & 0 & 2.917436 & -2.554506 & -0.758691 \\
\hline 2 & 7 & $\odot$ & 3.030947 & -1.162238 & -0.326406 \\
\hline 3 & 15 & $\odot$ & 2.233032 & 0.140092 & -1.064218 \\
\hline 4 & 15 & ○ & -2.168925 & $\odot .101596$ & -0.501172 \\
\hline 5 & 7 & ○ & -2.349958 & -0.151633 & 1.173520 \\
\hline 6 & 6 & $\odot$ & -1.236650 & -0.268210 & 2.143309 \\
\hline 7 & 7 & $\odot$ & 3.035714 & 1.197767 & -0.008651 \\
\hline 8 & 6 & 0 & 2.929939 & 2.655333 & -0.058438 \\
\hline 9 & 6 & 0 & 3.722534 & 0.537360 & 1.020358 \\
\hline 10 & 6 & 0 & 3.719821 & -0.800261 & 0.840216 \\
\hline 11 & 7 & $\odot$ & -3.870875 & 0.085496 & -0.636872 \\
\hline 12 & 6 & $\odot$ & -4.582899 & 0.260147 & -1.917247 \\
\hline 13 & 6 & $\odot$ & -4.530930 & -0.106091 & 0.542804 \\
\hline 14 & 6 & $\odot$ & -3.651231 & -0.241816 & 1.585273 \\
\hline 15 & 1 & $\odot$ & 4.195264 & -1.553893 & 1.451769 \\
\hline 16 & 1 & 0 & 4.200737 & 1.100499 & 1.809151 \\
\hline 17 & 9 & $\odot$ & 0.587394 & 0.034345 & -0.258666 \\
\hline 18 & 1 & $\odot$ & -5.611360 & -0.138050 & 0.586399 \\
\hline
\end{tabular}




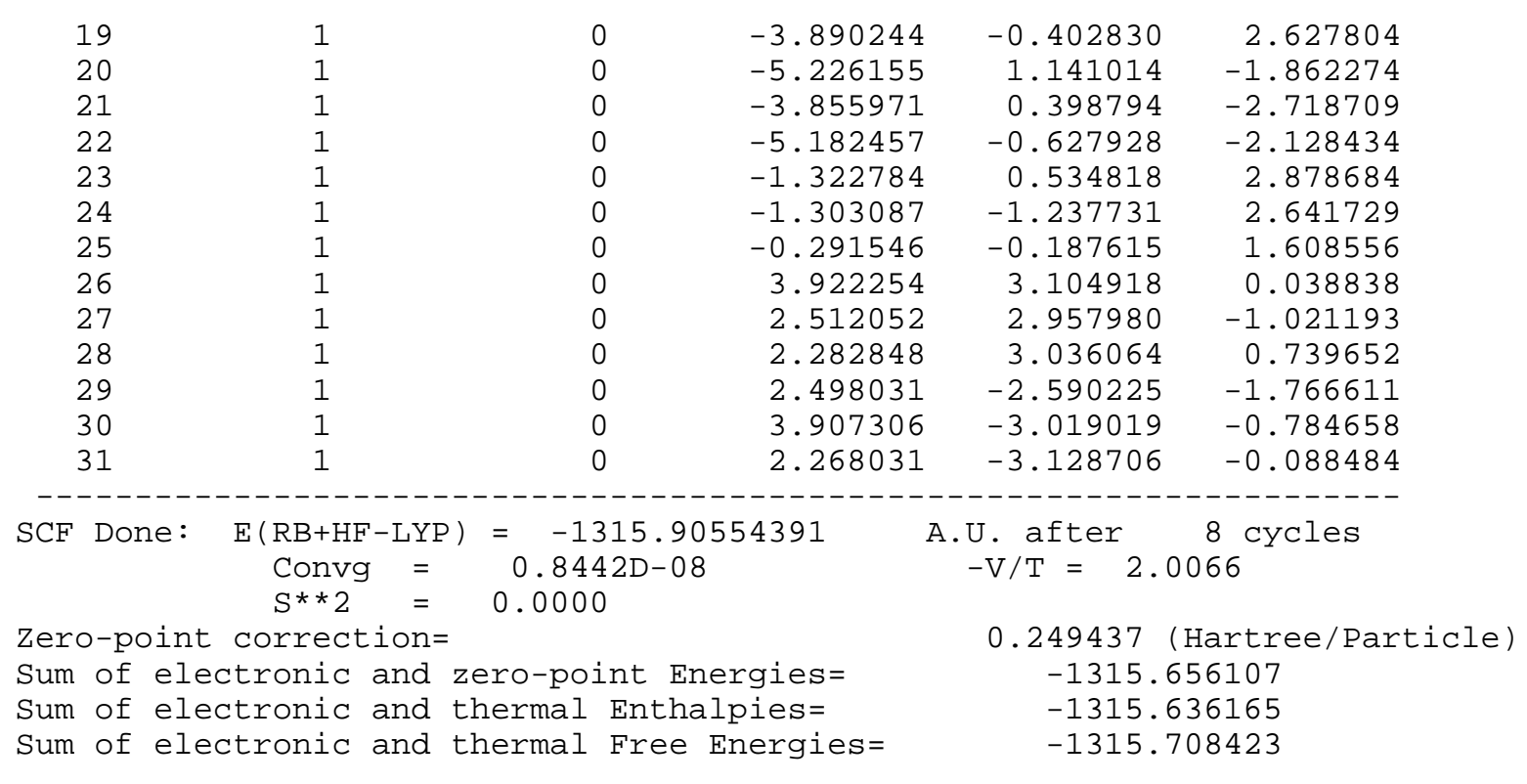

F-bridged 2-Fluoro-1,3-Dimethyl-1,3,2-diazaphospholene / 1,3-Dimethyl-1,3,2-

diazaphospholenium Complex $4 \mathrm{~b}[\mathrm{~F}]\left(\mathrm{C}_{2}\right.$-symmetric Transition State):

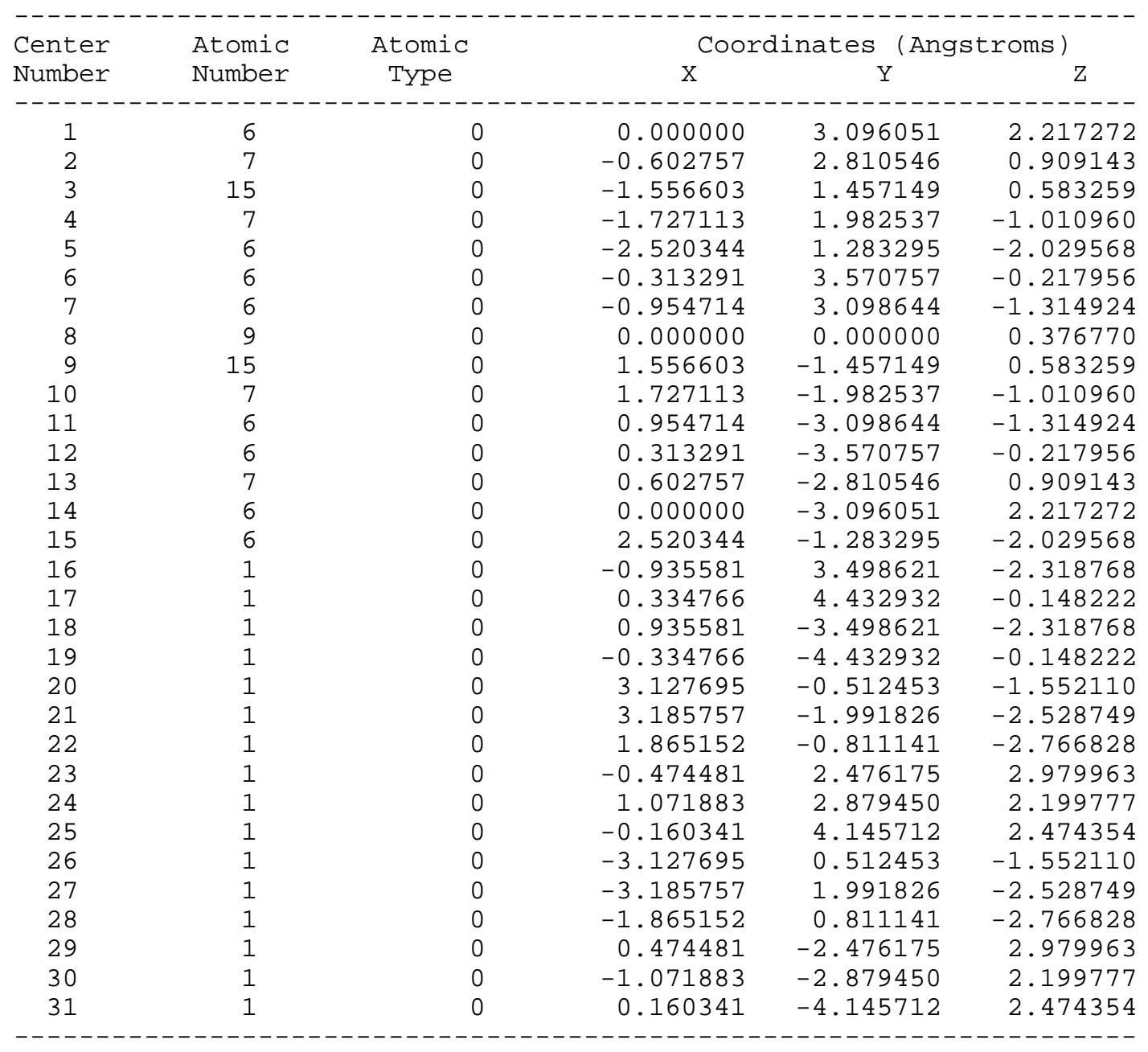




$$
\begin{aligned}
& \text { SCF Done: } E(R B+H F-L Y P)=-1315.90130394 \\
& \text { Convg }=0.9408 \mathrm{D}-08 \\
& \mathrm{~S}^{* * 2}=0.0000
\end{aligned}
$$

Zero-point correction=

Sum of electronic and zero-point Energies=

Sum of electronic and thermal Enthalpies=

Sum of electronic and thermal Free Energies=
A.U. after 1 cycles
$-\mathrm{V} / \mathrm{T}=2.0066$
๑.248676 (Hartree/Particle)
$-1315.652628$
$-1315.633048$
$-1315.703103$

\section{Cl-bridged 2-Chloro-1,3-Dimethyl-1,3,2-diazaphospholene / 1,3-Dimethyl-1,3,2-}

\section{diazaphospholenium Complex 4b[Cl]:}

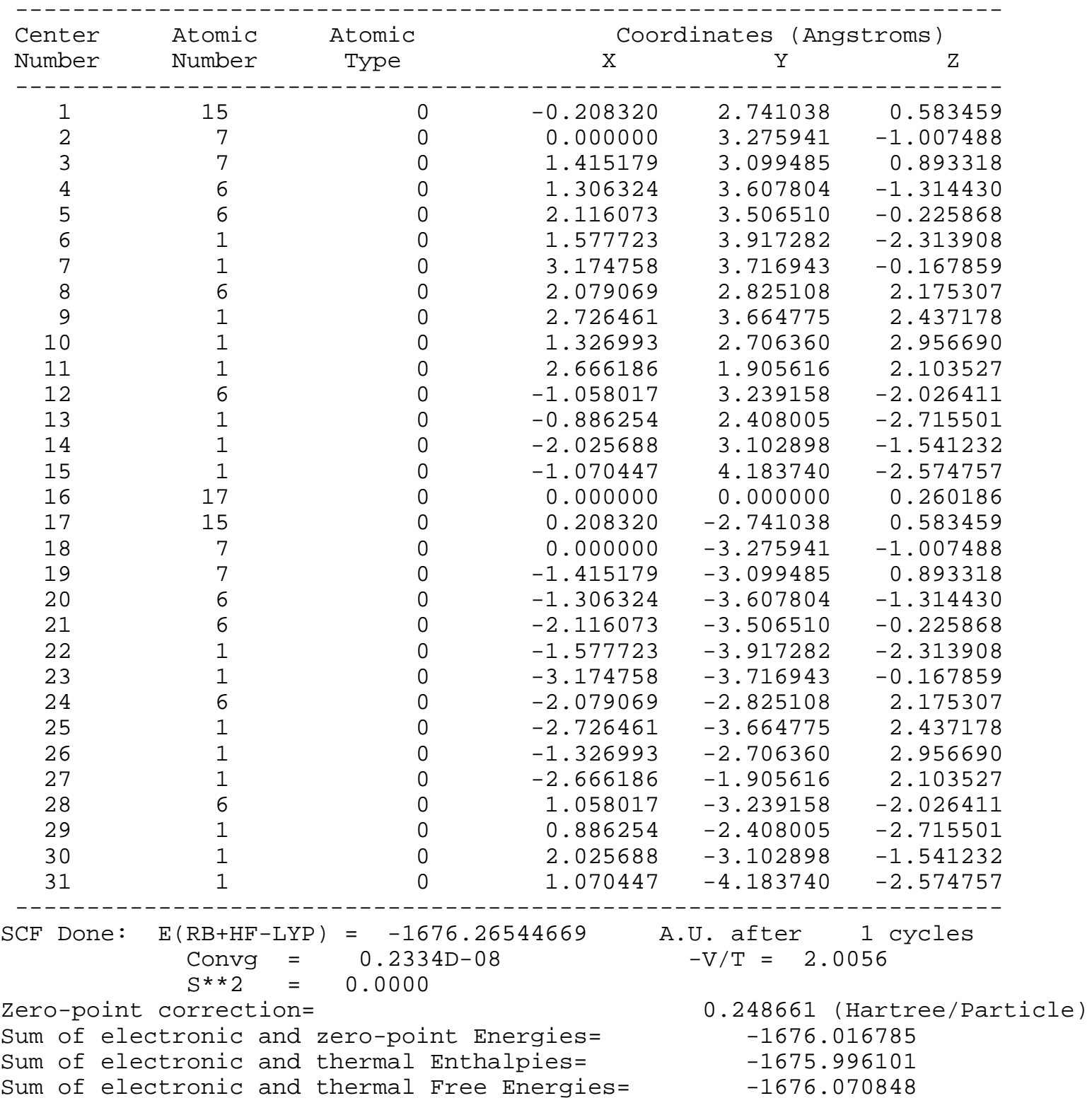

\section{Br-bridged 2-Bromo-1,3-Dimethyl-1,3,2-diazaphospholene / 1,3-Dimethyl-1,3,2-}

diazaphospholenium Complex 4b[Br]: 


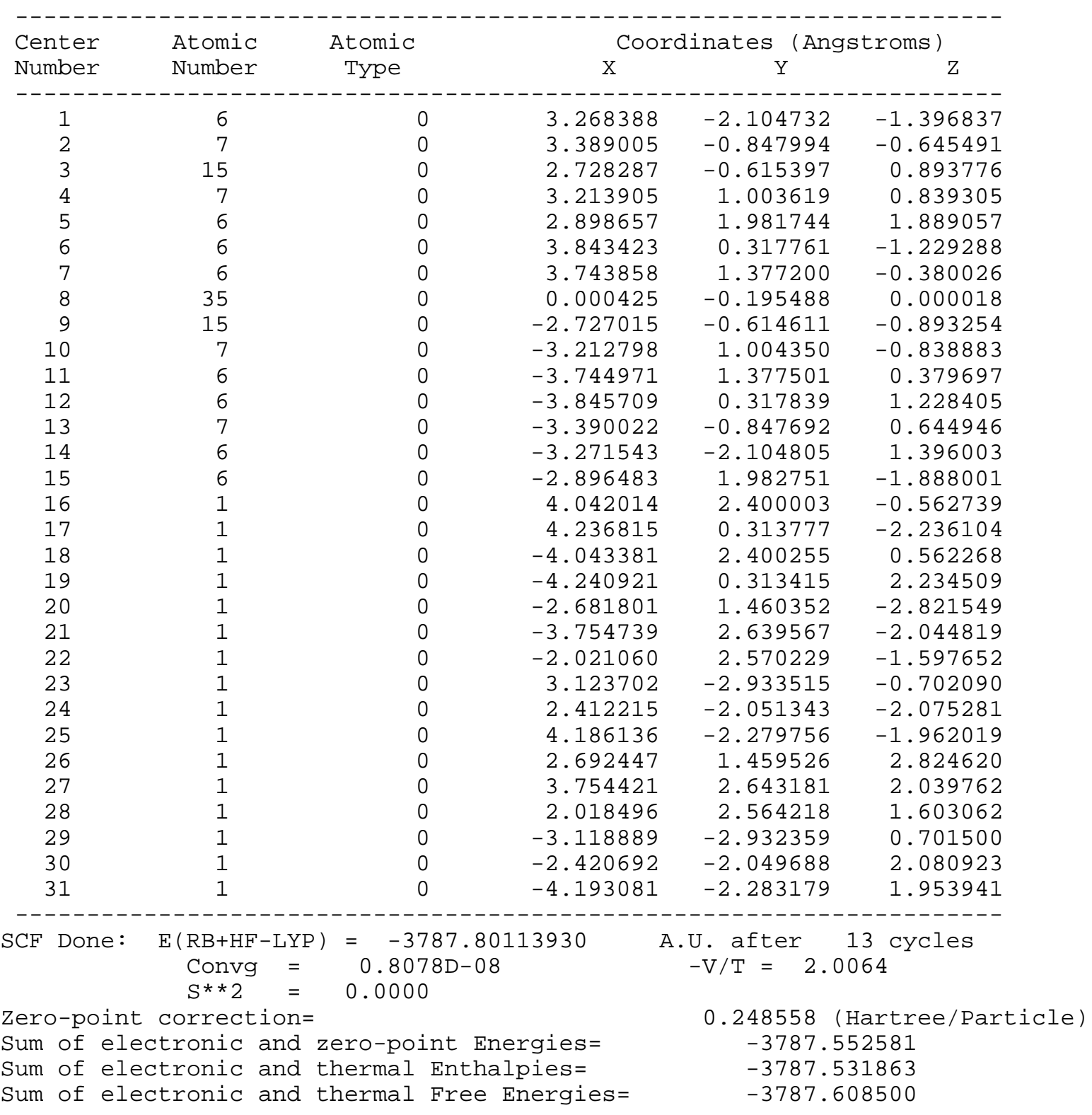

Cl-bridged 2-Chloro-1,3-Dimethyl-1,3,2-diazaphospholidine / 1,3-Dimethyl-1,3,2-

diazaphospholidinium Complex 5b[Cl]:

\begin{tabular}{|c|c|c|c|c|c|}
\hline \multirow{2}{*}{$\begin{array}{l}\text { Center } \\
\text { Number }\end{array}$} & Atomic & Atomic & \multicolumn{3}{|c|}{ Coordinates (Angstroms) } \\
\hline & Number & Type & $\mathrm{X}$ & $Y$ & Z \\
\hline & & & - - - - - - & --- & \\
\hline 1 & 6 & $\odot$ & 3.701846 & -1.273567 & -0.368928 \\
\hline 2 & 6 & $\odot$ & 3.994370 & -0.038821 & -1.235468 \\
\hline 3 & 7 & $\odot$ & 3.140578 & 1.041453 & -0.701523 \\
\hline 4 & 15 & $\odot$ & 2.424220 & 0.729085 & 0.753882 \\
\hline 5 & 17 & $\odot$ & 0.005160 & -0.057659 & -0.062156 \\
\hline 6 & 7 & $\odot$ & 3.153443 & -0.755070 & $\odot .900798$ \\
\hline 7 & 15 & ○ & -2.471033 & -0.745920 & $\odot .679999$ \\
\hline 8 & 7 & $\odot$ & -3.099028 & -0.997054 & -0.835906 \\
\hline 9 & 6 & $\odot$ & -3.606019 & 0.232820 & -1.477934 \\
\hline 10 & 6 & $\odot$ & -3.977438 & 1.183833 & -0.329599 \\
\hline
\end{tabular}




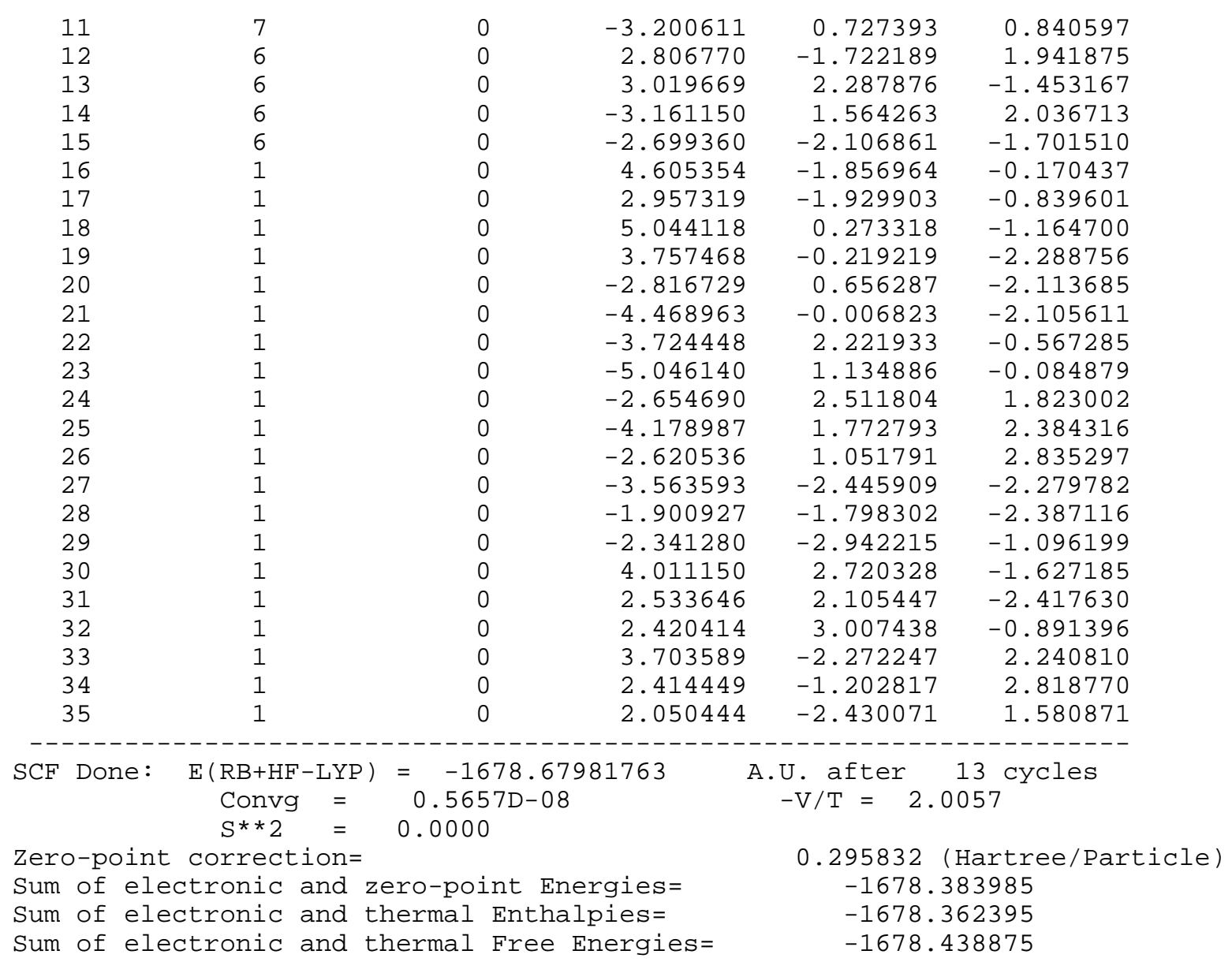

Cl-bridged Chloro-bis(dimethylamino)-phosphine / bis(dimethylamino)-phosphenium Complex

$6 \mathrm{~b}[\mathrm{Cl}]:$

\begin{tabular}{|c|c|c|c|c|c|}
\hline \multirow{3}{*}{$\begin{array}{l}\text { Center } \\
\text { Number }\end{array}$} & Atomic & Atomic & \multicolumn{3}{|c|}{ Coordinates (Angstroms) } \\
\hline & Number & Type & $x$ & $\mathrm{Y}$ & Z \\
\hline & & -- & ---- & 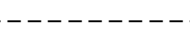 & $-\ldots$ \\
\hline 1 & 6 & $\odot$ & -2.971308 & 2.078330 & $\odot .081682$ \\
\hline 2 & 6 & 0 & -3.166424 & 1.194366 & 2.371784 \\
\hline 3 & 7 & $\odot$ & -3.031196 & $\odot .883305$ & $\odot .936212$ \\
\hline 4 & 6 & $\odot$ & -2.525392 & -2.226665 & -1.555335 \\
\hline 5 & 6 & 0 & -3.972050 & -0.240154 & -1.818719 \\
\hline 6 & 7 & $\odot$ & -3.093938 & -1.015996 & -0.938221 \\
\hline 7 & 15 & $\odot$ & -2.519775 & -0.655023 & ๑.577297 \\
\hline 8 & 15 & 0 & 2.476514 & 0.620695 & 0.647666 \\
\hline 9 & 7 & 0 & 3.082652 & 1.035631 & $-\odot .840815$ \\
\hline 10 & 6 & $\odot$ & 3.131855 & ๑. 218446 & -2.062547 \\
\hline 11 & 6 & $\odot$ & 3.216776 & 2.482545 & -1.096416 \\
\hline 12 & 7 & $\odot$ & 3. 038131 & -0.902306 & $\odot .99670 \odot$ \\
\hline 13 & 6 & 0 & 2.377843 & -1.567421 & 2.132253 \\
\hline 14 & 6 & 0 & 3.979718 & -1.749271 & $\odot .258831$ \\
\hline 15 & 17 & $\odot$ & -0.001025 & -0.079254 & -0.163587 \\
\hline 16 & 1 & 0 & -2.257429 & 2.786022 & $\odot .517577$ \\
\hline 17 & 1 & ○ & -3.952790 & 2.560762 & $\odot .020875$ \\
\hline 18 & 1 & $\odot$ & -2.622980 & 1.826771 & -0.917645 \\
\hline 19 & 1 & $\odot$ & -4.134824 & 1.672493 & 2.551458 \\
\hline 20 & 1 & $\odot$ & -2.367229 & 1.870435 & 2.695998 \\
\hline
\end{tabular}




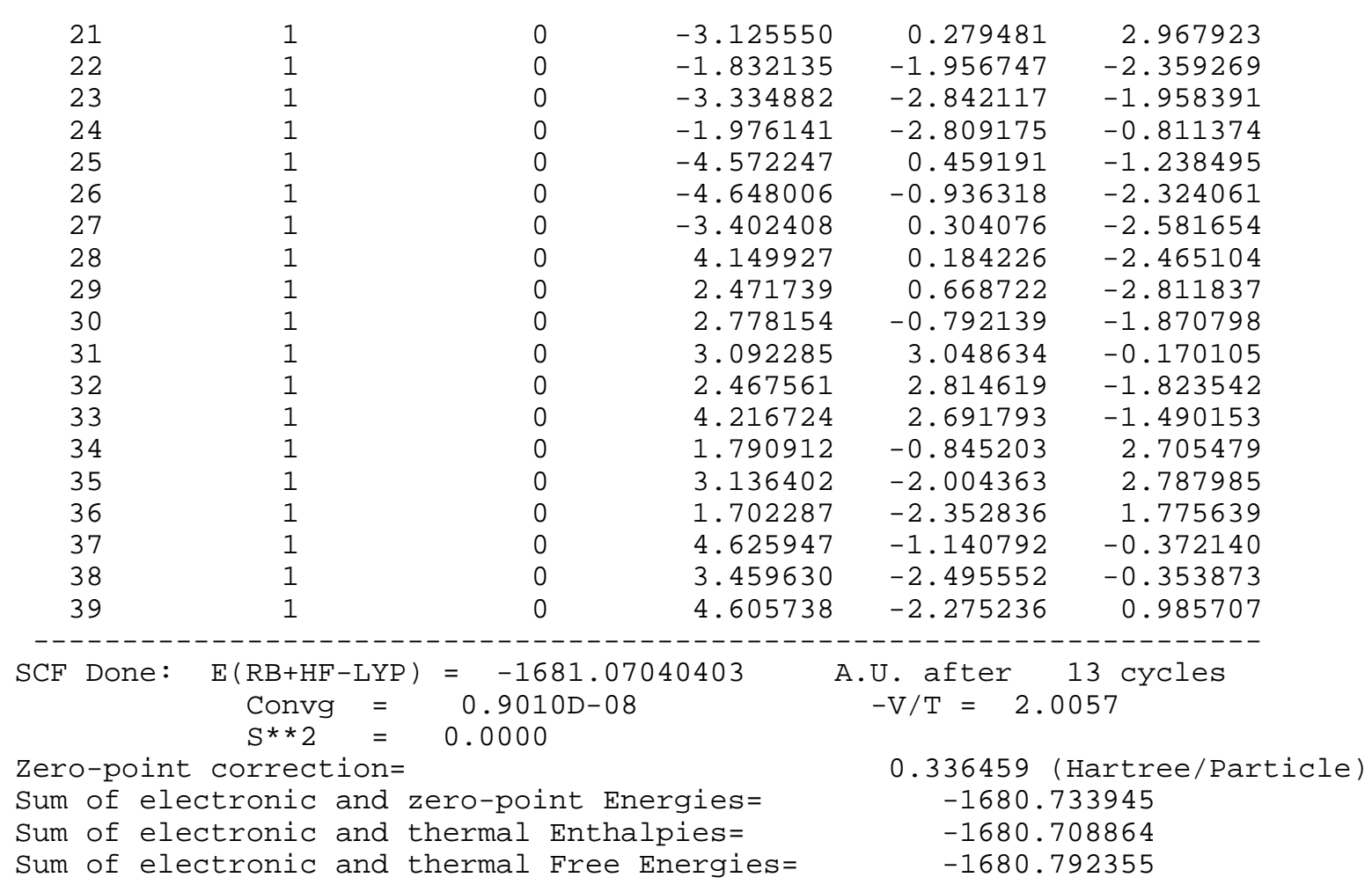

\section{P-P bonded Chloro-bis(dimethylamino)-phosphine / bis(dimethylamino)-phosphenium Complex}

\section{$6^{\prime} \mathrm{b}[\mathrm{Cl}]$}

\begin{tabular}{|c|c|c|c|c|c|}
\hline Center & Atomic & Atomic & Coor & nates (An) & roms ) \\
\hline Number & Number & Type & $x$ & Y & Z \\
\hline 1 & 7 & & 2123076 & 1 ค20251 & ค 660ค61 \\
\hline $\begin{array}{l}1 \\
2\end{array}$ & $\begin{array}{r}7 \\
15\end{array}$ & $\begin{array}{l}0 \\
0\end{array}$ & $\begin{array}{l}-2.423076 \\
-1.247336\end{array}$ & $\begin{array}{r}-1.028251 \\
0.058334\end{array}$ & $\begin{array}{l}-0.669064 \\
-0.191724\end{array}$ \\
\hline $\begin{array}{l}2 \\
3\end{array}$ & $\begin{array}{l}15 \\
15\end{array}$ & 8 & $\begin{array}{r}-1.241330 \\
2.056550\end{array}$ & $\begin{array}{r}0.058334 \\
-0.239019\end{array}$ & -1.094361 \\
\hline 4 & 7 & 8 & 2.401632 & -1361727 & 8061928 \\
\hline 5 & 17 & 0 & $-0 \quad 586073$ & -0 586916 & 1835219 \\
\hline 6 & 7 & 0 & 2220661 & 1300109 & 287037 \\
\hline 7 & 7 & 0 & 2476069 & 1261527 & 013616 \\
\hline 8 & 6 & 0 & 3668650 & 1278175 & \\
\hline 9 & & & & & . \\
\hline 10 & & & & & משנים \\
\hline & & & & & \\
\hline$\perp \perp$ & & U & & & $-0.138<34$ \\
\hline 12 & 6 & 0 & 2.495772 & 1.806861 & ๑.824588 \\
\hline 13 & 6 & $\odot$ & 1.821766 & -2.702764 & -0.167215 \\
\hline 14 & 6 & $\odot$ & 3.145959 & -1.247594 & 1.324973 \\
\hline 15 & 6 & $\odot$ & 2.686331 & 2.281458 & -1.594664 \\
\hline 16 & 1 & $\Theta$ & 2.456276 & -1.167607 & 2.171896 \\
\hline 17 & 1 & $\Theta$ & 3.747290 & -2.152978 & 1.443364 \\
\hline 18 & 1 & $\odot$ & 3.816837 & -0.391245 & 1.301116 \\
\hline 19 & 1 & $\odot$ & 1.072451 & -2.912454 & ๑. 601883 \\
\hline 20 & 1 & 0 & 1.344208 & -2.750097 & -1.149121 \\
\hline 21 & 1 & 0 & 2.618147 & -3.450559 & $-\odot .125598$ \\
\hline 22 & 1 & $\odot$ & 1.909491 & 2.731476 & $\odot .828468$ \\
\hline 23 & 1 & $\odot$ & 2.039107 & 1.113335 & 1.527117 \\
\hline 24 & 1 & 0 & 3.518174 & 2.044143 & 1.136297 \\
\hline 25 & 1 & 0 & 1.953396 & 3.086425 & -1.485238 \\
\hline 26 & 1 & 0 & 3.695300 & 2.695881 & -1.504653 \\
\hline
\end{tabular}




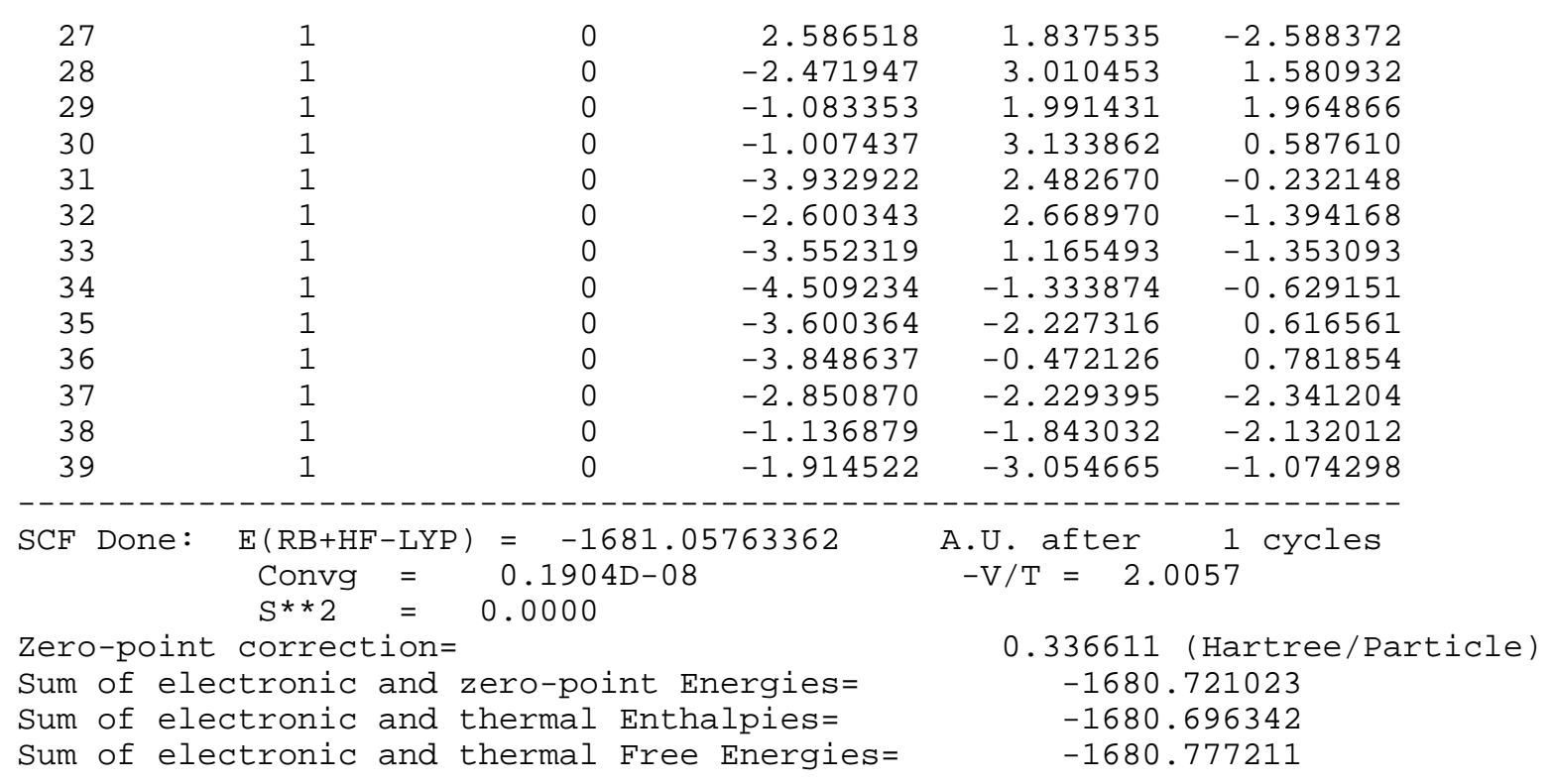

Final atomic coordinates and energies for gas phase structures at mpw1k/6-31+g** level:

\section{1,3-Dimethyl-1,3,2-diazaphospholenium cation $1 \mathrm{~b}$ :}

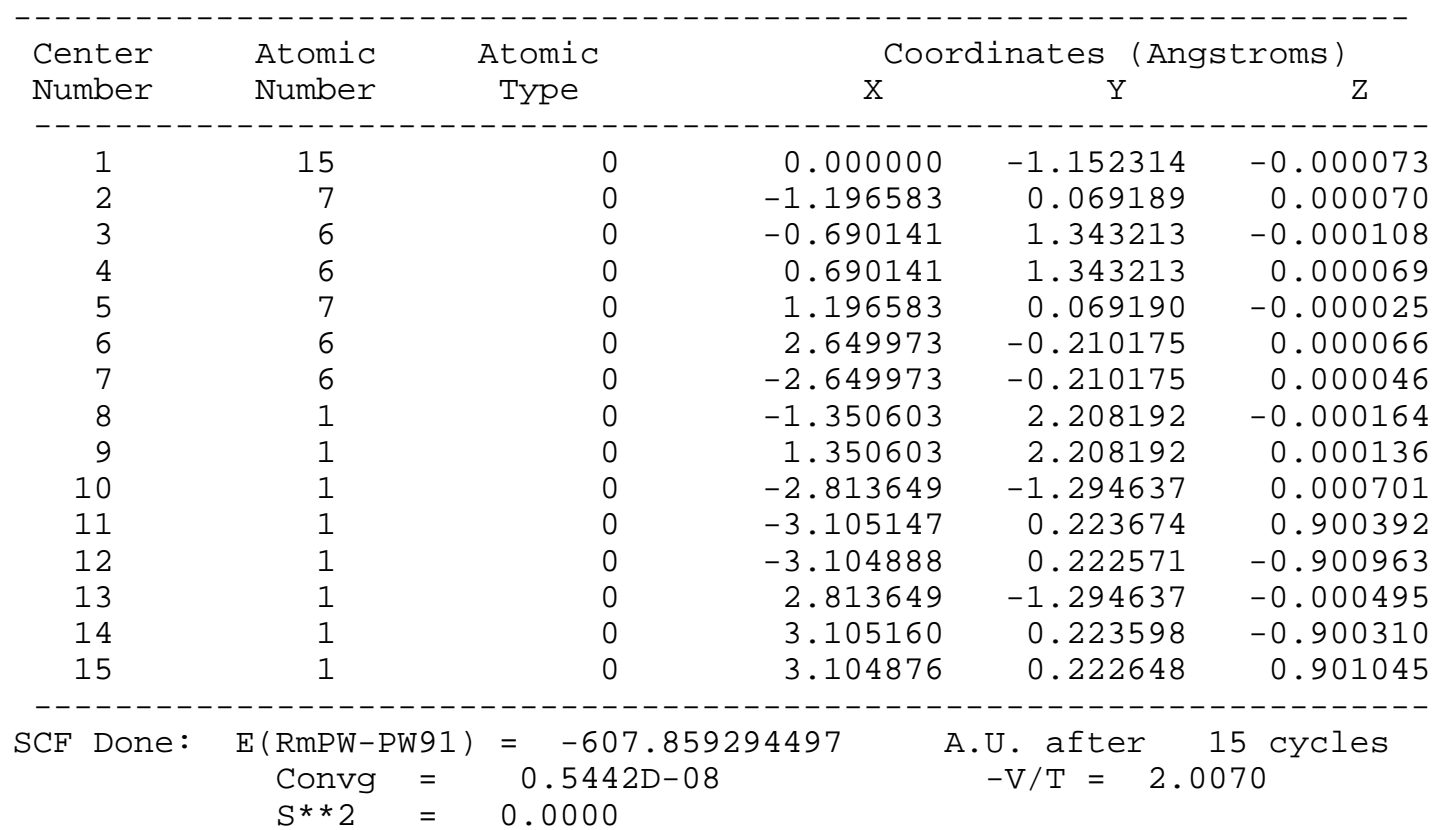

Bis-(Dimethylamino)-phosphenium cation 3b:

\begin{tabular}{ccccc} 
Center & Atomic & Atomic & \multicolumn{2}{c}{ Coordinates (Angstroms) } \\
Number & Number & Type & $X$ & $Y$
\end{tabular}




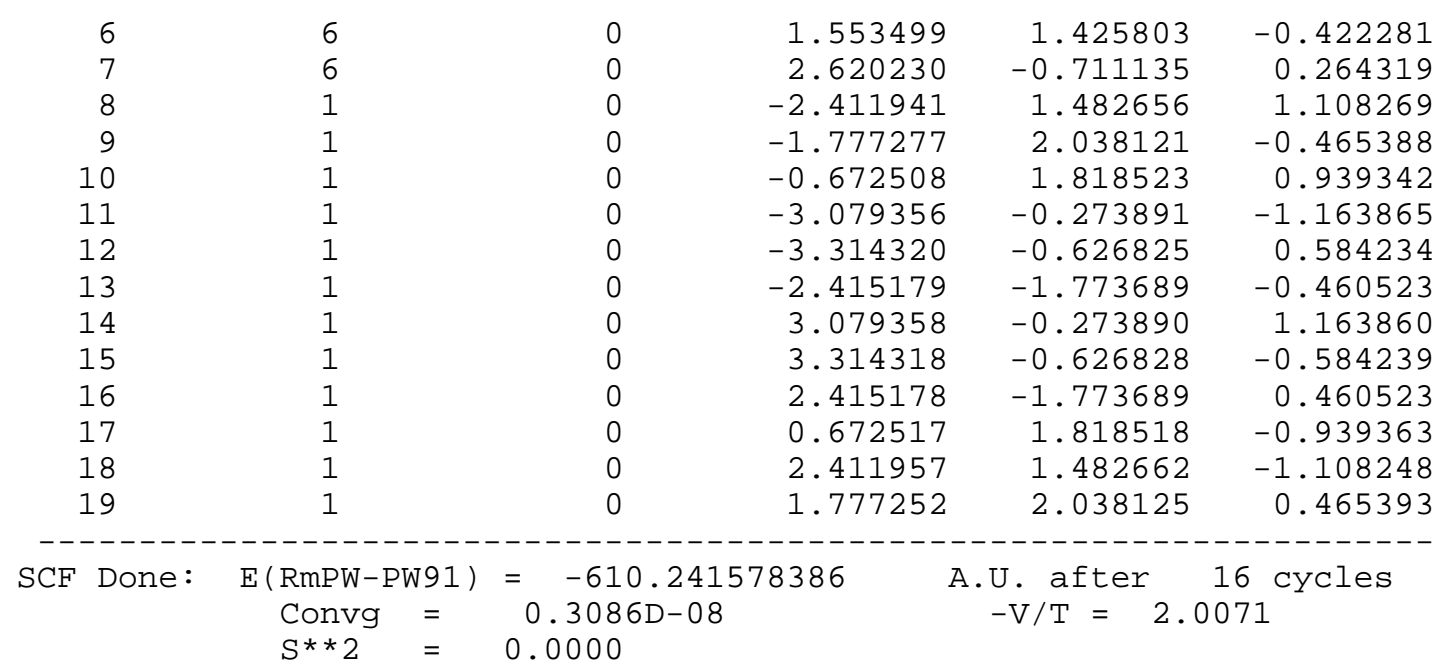

2-Fluoro-1,3-Dimethyl-1,3,2-diazaphospholene 1b[F]:

\begin{tabular}{|c|c|c|c|c|c|}
\hline \multirow{2}{*}{$\begin{array}{l}\text { Center } \\
\text { Number }\end{array}$} & Atomic & Atomic & \multicolumn{3}{|c|}{ Coordinates (Angstroms) } \\
\hline & Number & Tyре & $\mathrm{X}$ & Y & Z \\
\hline & & & & & \\
\hline 1 & 9 & ○ & -0.000490 & -1.760644 & $\odot .978882$ \\
\hline 2 & 15 & $\odot$ & -0.000010 & -0.857945 & -0.494265 \\
\hline 3 & 7 & ○ & -1.197227 & 0.346701 & -0.194330 \\
\hline 4 & 6 & ○ & -2.631319 & $\odot .083017$ & -0.256467 \\
\hline 5 & 7 & ○ & 1.197389 & $\odot .346396$ & -0.193992 \\
\hline 6 & 6 & ○ & 2.631455 & 0.082679 & -0.256447 \\
\hline 7 & 6 & ○ & 0.679460 & 1.517607 & $\odot .385211$ \\
\hline 8 & 6 & ○ & -0.679155 & 1.517732 & $\odot .385098$ \\
\hline 9 & 1 & $\odot$ & 1.347024 & 2.297300 & $\odot .743786$ \\
\hline 10 & 1 & $\odot$ & -1.346629 & 2.297545 & 0.743579 \\
\hline 11 & 1 & $\odot$ & 2.814982 & -0.783707 & $-\odot .907772$ \\
\hline 12 & 1 & $\odot$ & 3.156293 & 0.950446 & -0.686689 \\
\hline 13 & 1 & 0 & 3.052977 & -0.136192 & 0.739672 \\
\hline 14 & 1 & $\odot$ & -2.815114 & -0.782694 & -0.908616 \\
\hline 15 & 1 & $\odot$ & -3.052465 & -0.136835 & 0.739594 \\
\hline 16 & 1 & $\odot$ & -3.156291 & 0.951228 & -0.685636 \\
\hline SCF Done: & $E(R m P W-P W 91)$ & -70 & 28993 & U. after & 8 cycles \\
\hline & Convg = & 0.943 & & $-\mathrm{V} / \mathrm{T}=2 . \mathrm{C}$ & \\
\hline & $* 2$ & 0.000 & & & \\
\hline
\end{tabular}

2-Chloro-1,3-Dimethyl-1,3,2-diazaphospholene 1b[Cl]:

\begin{tabular}{|c|c|c|c|c|c|}
\hline Center & Atomic & Atomic & \multicolumn{3}{|c|}{ Coordinates (Angstroms) } \\
\hline Number & Number & Type & $x$ & $Y$ & Z \\
\hline 1 & 15 & & & & \\
\hline 1 & 15 & $\checkmark$ & -0.396303 & $0.85<600$ & 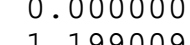 \\
\hline 2 & 1 & 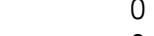 & $-0.66 / 226$ & -0.354121 & 1.199009 \\
\hline 3 & 7 & 0 & -0.667226 & -0.354121 & -1.199009 \\
\hline 4 & 6 & $\odot$ & $-\odot .667226$ & -1.654332 & $\odot .680320$ \\
\hline 5 & 6 & $\odot$ & -0.667226 & -1.654332 & -0.680320 \\
\hline 6 & 6 & 0 & -0.468136 & -0.093581 & 2.624346 \\
\hline 7 & 6 & $\odot$ & -0.468136 & -0.093581 & -2.624346 \\
\hline 8 & 1 & $\odot$ & $-\odot .70 \odot 620$ & -2.512791 & 1.346636 \\
\hline 9 & 1 & $\odot$ & -0.700620 & -2.512791 & -1.346636 \\
\hline 10 & 1 & $\odot$ & -0.727704 & 0.950803 & 2.845854 \\
\hline
\end{tabular}




\begin{tabular}{|c|c|c|c|c|c|}
\hline 11 & 1 & $\odot$ & -1.130138 & -0.745515 & 3.213421 \\
\hline 12 & 1 & $\odot$ & 0.579911 & -0.266504 & 2.919877 \\
\hline 13 & 1 & 0 & -0.727704 & 0.950803 & -2.845854 \\
\hline 14 & 1 & $\odot$ & 0.579911 & -0.266504 & -2.919877 \\
\hline 15 & 1 & $\odot$ & -1.130138 & -0.745515 & -3.213421 \\
\hline 16 & 17 & $\odot$ & 1.933361 & 1.075980 & $\odot .0 \odot \odot \odot \odot \odot$ \\
\hline Done & $\begin{array}{c}\mathrm{E}(\mathrm{RmPW}-\mathrm{PW} 91) \\
\text { Convg }= \\
\mathrm{S}^{*} 2=\end{array}$ & $\begin{array}{c}=\quad-106 \\
0.4052 \\
0.0000\end{array}$ & 97348 & $\begin{array}{l}\text { A.U. after } \\
-\mathrm{V} / \mathrm{T}=2\end{array}$ & 8 cycles \\
\hline
\end{tabular}

Chloro-bis-(dimethylamino)-phosphine $3 \mathrm{~b}[\mathrm{Cl}]$ :

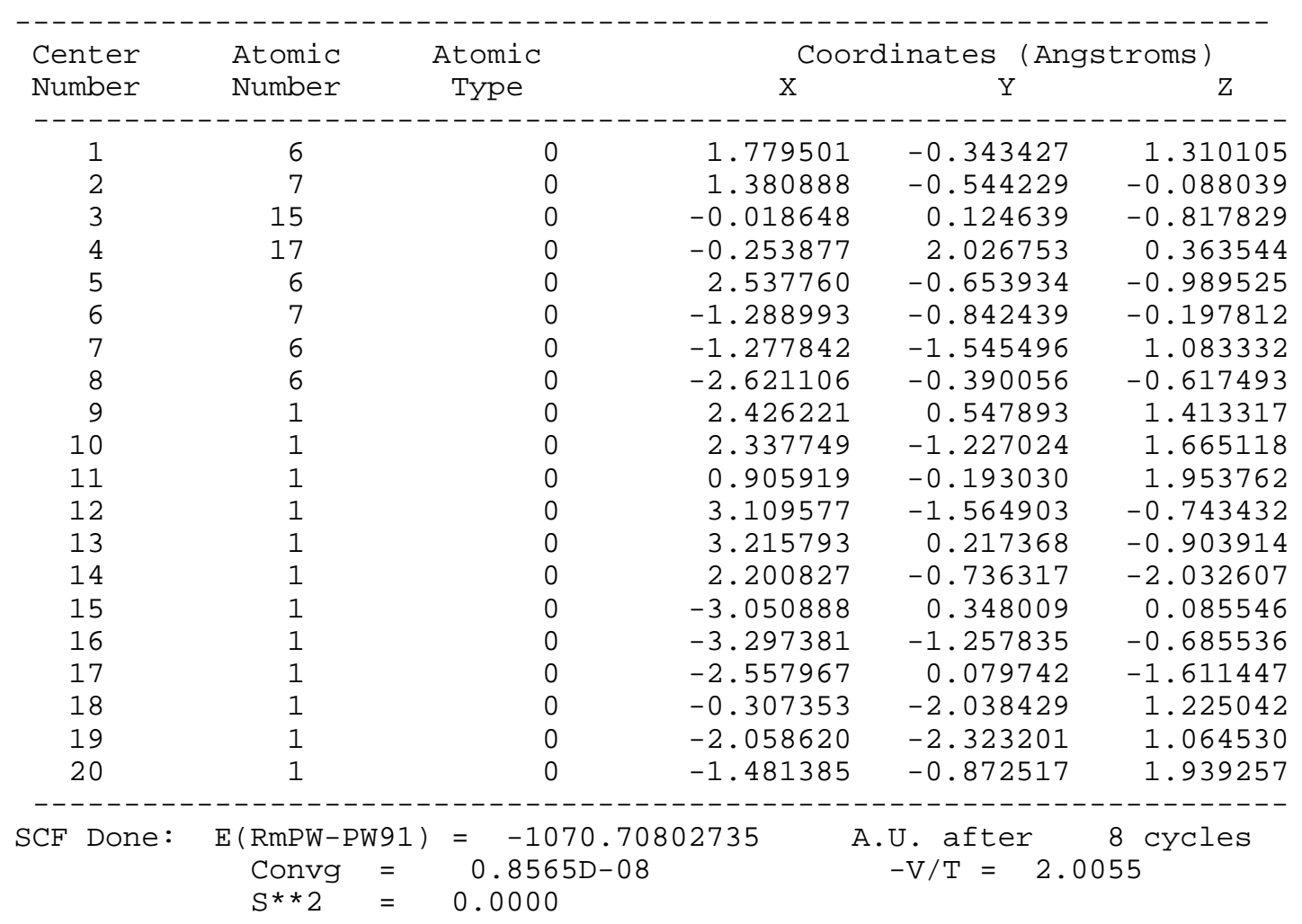

\section{F-bridged 2-Fluoro-1,3-Dimethyl-1,3,2-diazaphospholene / 1,3-Dimethyl-1,3,2-}

\section{diazaphospholenium Complex 4'b[F]:}

\begin{tabular}{|c|c|c|c|c|c|}
\hline \multirow{2}{*}{$\begin{array}{l}\text { Center } \\
\text { Number }\end{array}$} & \multirow{2}{*}{$\begin{array}{l}\text { Atomic } \\
\text { Number }\end{array}$} & Atomic & \multicolumn{3}{|c|}{ Coordinates (Angstroms) } \\
\hline & & Type & $X$ & Y & Z \\
\hline & & $-\cdots$ & $-\cdots$ & --- & $-\cdots----$ \\
\hline 1 & 6 & $\odot$ & -2.882042 & -1.834860 & 1.927276 \\
\hline 2 & 7 & $\odot$ & -3.018054 & -0.834517 & $\odot .864430$ \\
\hline 3 & 15 & 0 & -2.180014 & $\odot .653642$ & $\odot .845391$ \\
\hline 4 & 15 & 0 & 2.093165 & 0.312975 & $\odot .389836$ \\
\hline 5 & 7 & $\odot$ & 2.334401 & -0.757399 & -0.939302 \\
\hline 6 & 6 & 0 & 1.254526 & -1.393206 & -1.726713 \\
\hline 7 & 7 & 0 & -3.010208 & 1.058384 & -0.591278 \\
\hline 8 & 6 & $\odot$ & -2.865870 & 2.342195 & -1.283933 \\
\hline 9 & 6 & $\odot$ & -3.748907 & -0.001460 & -1.131172 \\
\hline 10 & 6 & 0 & -3.753384 & -1.080348 & -0.301402 \\
\hline 11 & 7 & ○ & 3.815727 & 0.419194 & $\odot .517349$ \\
\hline 12 & 6 & 0 & 4.487509 & 1.243883 & 1.538850 \\
\hline
\end{tabular}




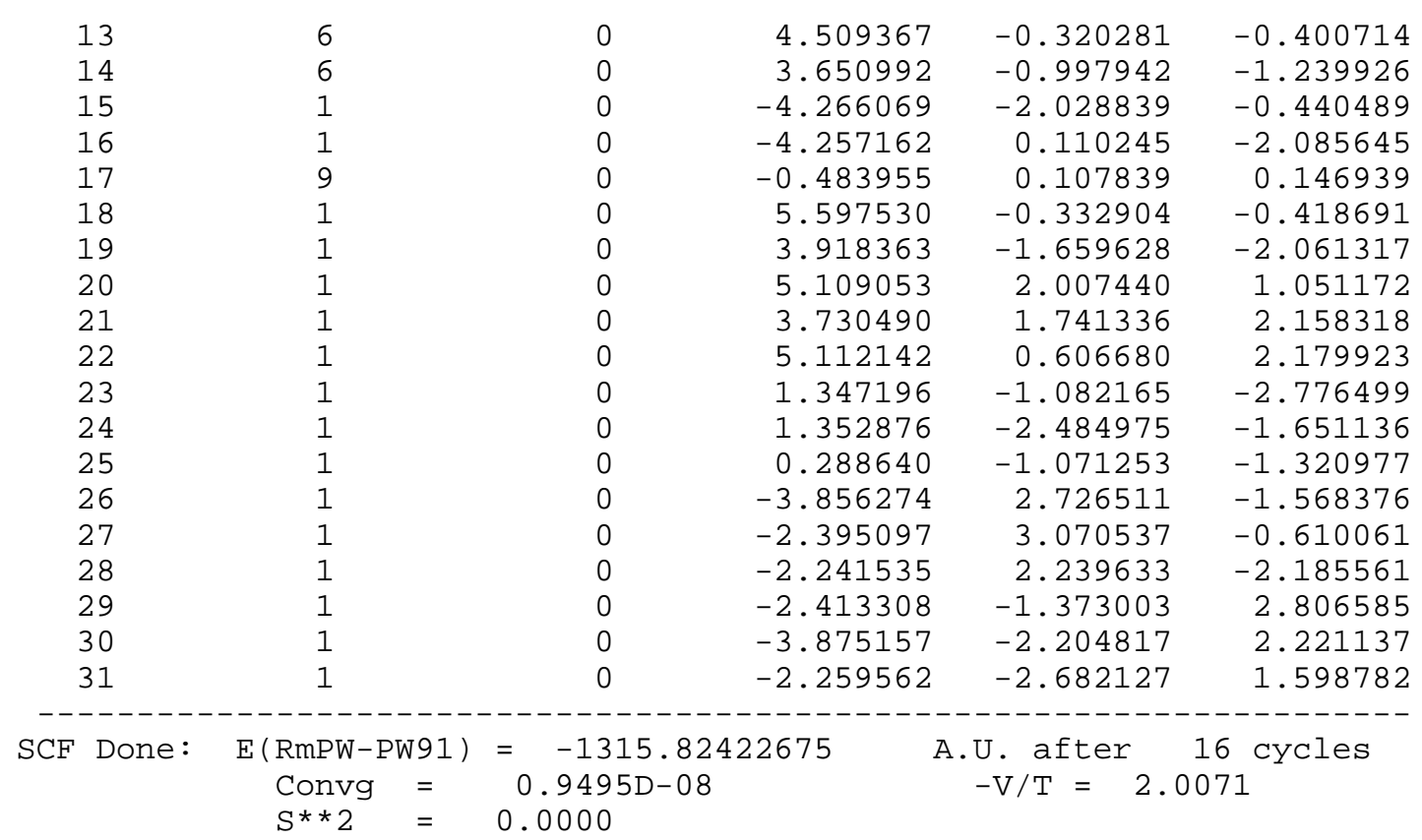

F-bridged 2-Fluoro-1,3-Dimethyl-1,3,2-diazaphospholene / 1,3-Dimethyl-1,3,2-

diazaphospholenium Complex $4 \mathrm{~b}[\mathrm{~F}]\left(\mathrm{C}_{2}\right.$-symmetric Transition State):

\begin{tabular}{|c|c|c|c|c|c|}
\hline \multirow{2}{*}{$\begin{array}{l}\text { Center } \\
\text { Number }\end{array}$} & Atomic & Atomic & \multicolumn{3}{|c|}{ Coordinates (Angstroms) } \\
\hline & Number & Type & $\mathrm{X}$ & Y & Z \\
\hline & & & & & 10 ח- \\
\hline 1 & 6 & 0 & $\odot . ๑ \odot \odot \odot \odot \odot$ & 3. 125617 & 2.208765 \\
\hline 2 & 7 & 0 & -0.615272 & 2.843737 & $\odot .903923$ \\
\hline 3 & 15 & $\odot$ & -1.562122 & 1.461807 & $\odot .587627$ \\
\hline 4 & 7 & 0 & -1.757689 & 2.006359 & -1.015707 \\
\hline 5 & 6 & 0 & -2.558999 & 1.298825 & -2.024500 \\
\hline 6 & 6 & $\odot$ & -0.357327 & 3.618143 & -0.221432 \\
\hline 7 & 6 & $\odot$ & -1.010253 & 3.139182 & -1.321274 \\
\hline 8 & 9 & 0 & $\odot ., \diamond \diamond \diamond \diamond \diamond \odot$ & $\odot ., \odot \odot \odot \odot \odot \odot ~$ & ๑. 399307 \\
\hline 9 & 15 & 0 & 1.562122 & -1.461807 & $\odot .587627$ \\
\hline 10 & 7 & $\odot$ & 1.757689 & -2.006359 & -1.015707 \\
\hline 11 & 6 & 0 & 1.010253 & -3.139182 & -1.321274 \\
\hline 12 & 6 & 0 & ๑. 357327 & -3.618143 & -0.221432 \\
\hline 13 & 7 & 0 & $\odot .615272$ & -2.843737 & 0.903923 \\
\hline 14 & 6 & $\odot$ & $\odot .00 \odot \odot \odot \odot$ & -3.125617 & 2.208765 \\
\hline 15 & 6 & 0 & 2.558999 & -1.298825 & -2.024500 \\
\hline 16 & 1 & 0 & -1.012436 & 3.550336 & -2.327701 \\
\hline 17 & 1 & $\odot$ & ๑. 278780 & 4.497305 & -0.154108 \\
\hline 18 & 1 & 0 & 1.012436 & -3.550336 & -2.327701 \\
\hline 19 & 1 & 0 & -0.278780 & -4.497305 & -0.154108 \\
\hline 20 & 1 & 0 & 3.144652 & -0.508587 & -1.537565 \\
\hline 21 & 1 & $\odot$ & 3.252369 & -2.001613 & -2.507440 \\
\hline 22 & 1 & 0 & 1.906943 & -0.841566 & -2.783129 \\
\hline 23 & 1 & 0 & -0.465928 & 2.495193 & 2.976869 \\
\hline 24 & 1 & 0 & 1.079091 & 2.913928 & 2.179500 \\
\hline 25 & 1 & 0 & -0.164891 & 4.178754 & 2.475413 \\
\hline 26 & 1 & 0 & -3.144652 & 0.508587 & -1.537565 \\
\hline 27 & 1 & 0 & -3.252369 & 2.001613 & -2.507440 \\
\hline 28 & 1 & 0 & -1.906943 & 0.841566 & -2.783129 \\
\hline 29 & 1 & $\odot$ & 0.465928 & -2.495193 & 2.976869 \\
\hline 30 & 1 & 0 & -1.079091 & -2.913928 & 2.179500 \\
\hline 31 & 1 & 0 & 0.164891 & -4.178754 & 2.475413 \\
\hline
\end{tabular}




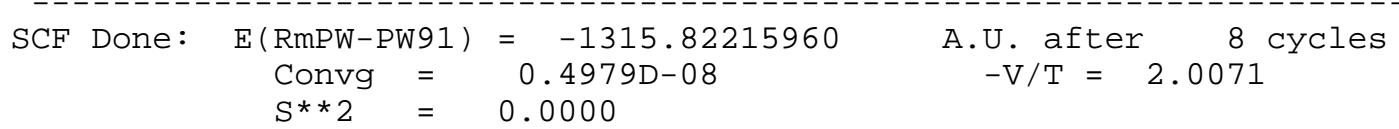

Cl-bridged 2-Chloro-1,3-Dimethyl-1,3,2-diazaphospholene / 1,3-Dimethyl-1,3,2diazaphospholenium Complex 4b[Cl]:

\begin{tabular}{|c|c|c|c|c|c|}
\hline \multirow{2}{*}{$\begin{array}{l}\text { Center } \\
\text { Number }\end{array}$} & \multirow{2}{*}{$\begin{array}{l}\text { Atomic } \\
\text { Number }\end{array}$} & Atomic & \multicolumn{3}{|c|}{ Coordinates (Angstroms) } \\
\hline & & \multirow[t]{2}{*}{ Type } & \multicolumn{2}{|r|}{ Y } & \multirow[t]{2}{*}{ Z } \\
\hline & & & & & \\
\hline 1 & 15 & $\odot$ & -0.222262 & 2.679922 & $\odot .568501$ \\
\hline 2 & 7 & $\odot$ & 0.000000 & 3.250876 & -1.027230 \\
\hline 3 & 7 & 0 & 1.406062 & 3.082168 & $\odot .897765$ \\
\hline 4 & 6 & $\odot$ & 1.303801 & 3.618972 & -1.314289 \\
\hline 5 & 6 & $\odot$ & 2.108759 & 3.522200 & -0.210627 \\
\hline 6 & 1 & $\odot$ & 1.580308 & 3.953232 & -2.311342 \\
\hline 7 & 1 & 0 & 3.166853 & 3.761927 & -0.137282 \\
\hline 8 & 6 & 0 & 2.058023 & 2.805916 & 2.186441 \\
\hline 9 & 1 & 0 & 2.707268 & 3.649592 & 2.457087 \\
\hline 10 & 1 & 0 & 1.294427 & 2.687687 & 2.965460 \\
\hline 11 & 1 & $\odot$ & 2.649359 & 1.880790 & 2.121449 \\
\hline 12 & 6 & $\odot$ & -1.052691 & 3. 213136 & -2.052855 \\
\hline 13 & 1 & $\odot$ & -0.860822 & 2.396954 & -2.764548 \\
\hline 14 & 1 & 0 & -2.025025 & 3.045256 & -1.572816 \\
\hline 15 & 1 & 0 & -1.084322 & 4.174212 & -2.584339 \\
\hline 16 & 17 & 0 & ๑. . 000000 & 0.000000 & ๑. 300359 \\
\hline 17 & 15 & $\odot$ & ๑. 222262 & -2.679922 & ๑.568501 \\
\hline 18 & 7 & 0 & 0.000000 & -3.250876 & -1.027230 \\
\hline 19 & 7 & $\odot$ & -1.406062 & -3.082168 & ๑.897765 \\
\hline 20 & 6 & $\odot$ & -1.303801 & -3.618972 & -1.314289 \\
\hline 21 & 6 & 0 & -2.108759 & -3.522200 & -0.210627 \\
\hline 22 & 1 & 0 & -1.580308 & -3.953232 & -2.311342 \\
\hline 23 & 1 & 0 & -3.166853 & -3.761927 & -0.137282 \\
\hline 24 & 6 & $\odot$ & -2.058023 & -2.805916 & 2.186441 \\
\hline 25 & 1 & 0 & -2.707268 & -3.649592 & 2.457087 \\
\hline 26 & 1 & $\odot$ & -1.294427 & -2.687687 & 2.965460 \\
\hline 27 & 1 & 0 & -2.649359 & -1.880790 & 2.121449 \\
\hline 28 & 6 & $\odot$ & 1.052691 & -3.213136 & -2.052855 \\
\hline 29 & 1 & $\odot$ & 0.860822 & -2.396954 & -2.764548 \\
\hline 30 & 1 & 0 & 2.025025 & -3.045256 & -1.572816 \\
\hline 31 & 1 & $\odot$ & 1.084322 & -4.174212 & -2.584339 \\
\hline & &.-- & ----- & --- & ------ \\
\hline SCF Done: & $\mathrm{E}$ (RmPW-PW91) & $=-167$ & 21549 & U. after & 8 cycles \\
\hline & Convg = & ( ) & & $-\mathrm{V} / \mathrm{T}=2$ & 61 \\
\hline & $S^{* * 2}$ & $\odot . \odot \odot \odot \odot$ & & & \\
\hline
\end{tabular}

Cl-bridged Chloro-bis(dimethylamino)-phosphine / bis(dimethylamino)-phosphenium Complex 6b[Cl]:

\begin{tabular}{|c|c|c|c|c|c|}
\hline \multirow{2}{*}{$\begin{array}{l}\text { Center } \\
\text { Number }\end{array}$} & \multirow{2}{*}{$\begin{array}{l}\text { Atomic } \\
\text { Number }\end{array}$} & Atomic & \multicolumn{3}{|c|}{ Coordinates (Angstroms) } \\
\hline & & Type & 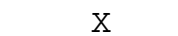 & $Y$ & Z \\
\hline & & -- & 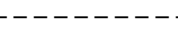 & --- & - - - - - - - \\
\hline 1 & 6 & $\odot$ & 2.919415 & -0.455776 & 2.037837 \\
\hline 2 & 6 & $\odot$ & 3.178123 & 1.984213 & 1.756329 \\
\hline 3 & 7 & $\odot$ & 3. 010198 & $\odot .673416$ & 1.099889 \\
\hline 4 & 6 & $\odot$ & 2.503588 & -0.949272 & -2.551819 \\
\hline
\end{tabular}




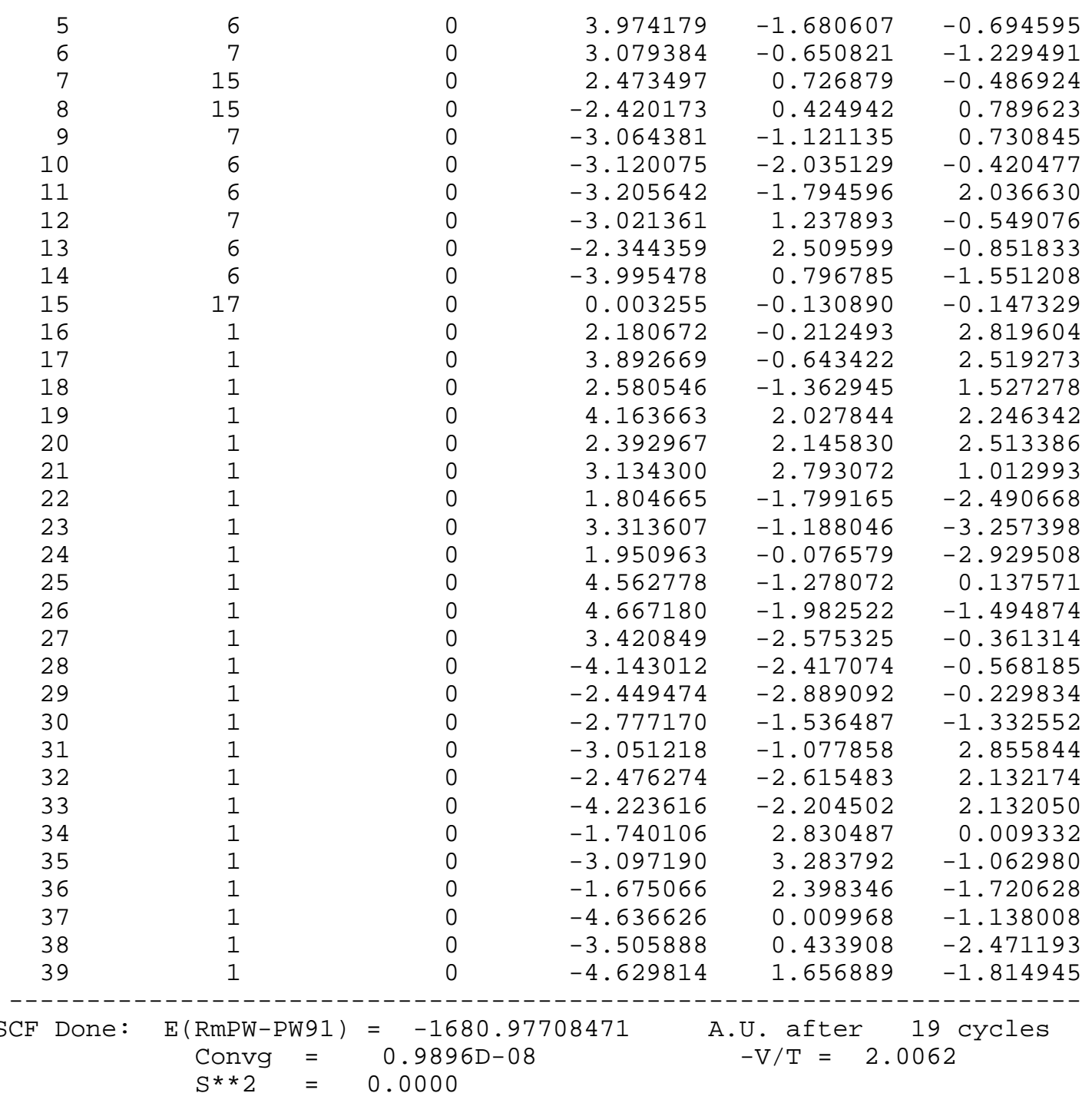

\section{P-P bonded Chloro-bis(dimethylamino)-phosphine / bis(dimethylamino)-phosphenium Complex}

\section{6'b[Cl]:}

\begin{tabular}{|c|c|c|c|c|c|}
\hline \multirow{2}{*}{$\begin{array}{l}\text { Center } \\
\text { Number }\end{array}$} & Atomic & Atomic & \multicolumn{3}{|c|}{ Coordinates (Angstroms) } \\
\hline & Number & Type & $x$ & Y & Z \\
\hline & & & $-\cdots-$ & ------ & 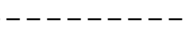 \\
\hline 1 & 7 & $\odot$ & -2.148612 & $-\odot .969685$ & $-\odot .873532$ \\
\hline 2 & 15 & $\odot$ & -1.047528 & -0.015205 & -0.038685 \\
\hline 3 & 15 & $\odot$ & 1.728603 & -0.210719 & -1.045899 \\
\hline 4 & 7 & $\odot$ & 2.330268 & -1.269289 & 0.106491 \\
\hline 5 & 17 & $\odot$ & -0.710320 & -0.873307 & 1.933725 \\
\hline 6 & 7 & $\odot$ & -2.054374 & 1.309259 & 0.404133 \\
\hline 7 & 7 & $\odot$ & 2.219576 & 1.330933 & $-\odot .601836$ \\
\hline 8 & 6 & $\odot$ & -3.515567 & -1.239550 & $-\odot .393001$ \\
\hline 9 & 6 & $\odot$ & -1.664070 & -1.925127 & -1.880026 \\
\hline 10 & 6 & $\odot$ & -1.753803 & 2.171414 & 1.555151 \\
\hline 11 & 6 & $\odot$ & -2.691907 & 2.052393 & $-\odot .702008$ \\
\hline 12 & 6 & $\odot$ & 2.167641 & 1.966668 & 0.723120 \\
\hline 13 & 6 & 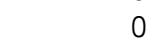 & 1.867122 & -2.664426 & -0.012889 \\
\hline 14 & 6 & $\odot$ & 3.183921 & -1.005623 & 1.270671 \\
\hline 15 & 6 & $\odot$ & 2.409905 & 2.281872 & -1.715682 \\
\hline
\end{tabular}




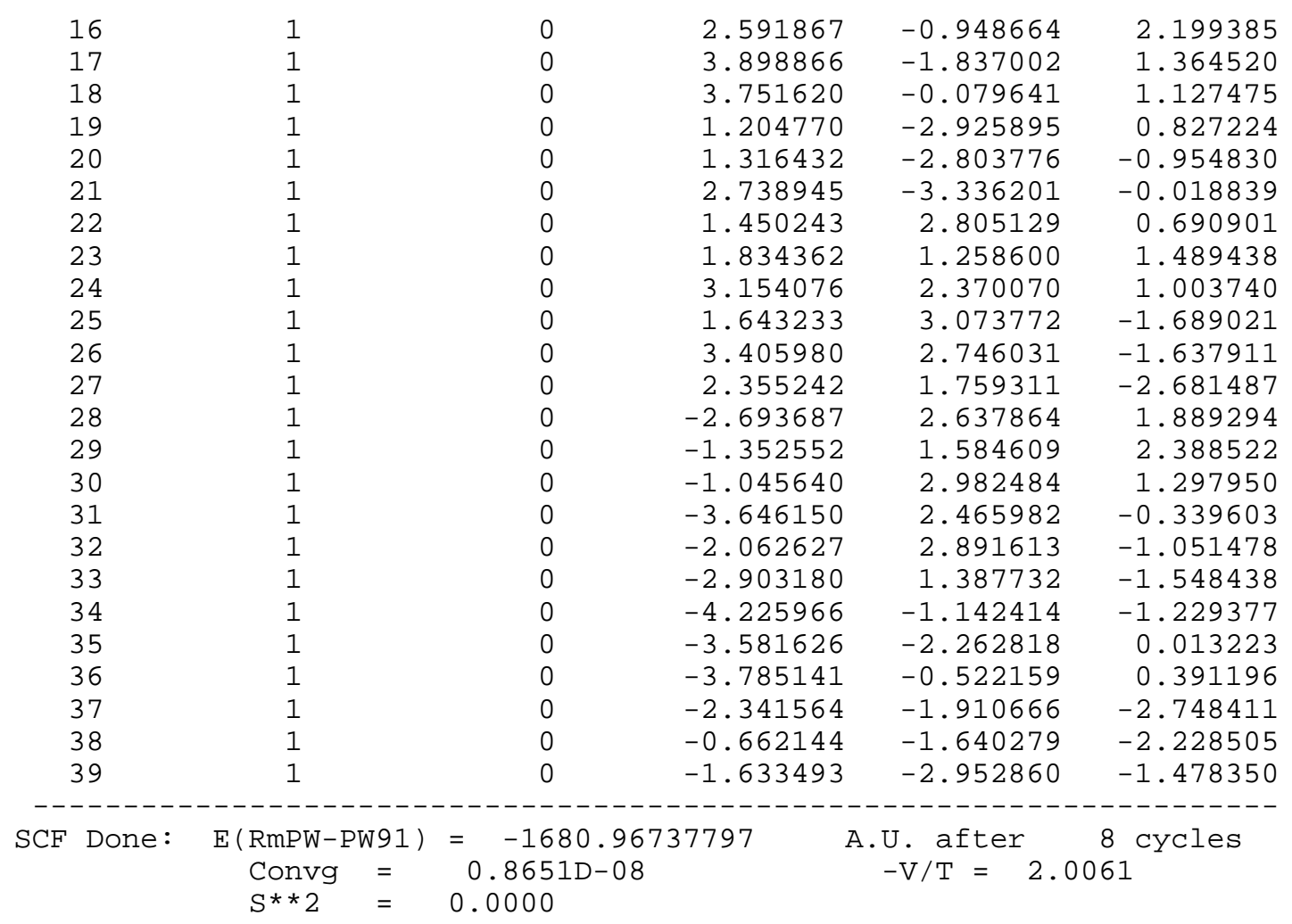

Final atomic coordinates and energies for gas phase structures at $\mathrm{mp} 2 / 6-31+\mathrm{g} * *$ level:

\section{1,3-Dimethyl-1,3,2-diazaphospholenium cation $1 \mathrm{~b}$ :}

\begin{tabular}{|c|c|c|c|c|c|}
\hline \multirow{2}{*}{$\begin{array}{l}\text { Center } \\
\text { Number }\end{array}$} & \multirow{2}{*}{$\begin{array}{l}\text { Atomic } \\
\text { Number }\end{array}$} & \multirow{2}{*}{$\begin{array}{c}\text { Atomic } \\
\text { Type }\end{array}$} & \multicolumn{3}{|c|}{ Coordinates (Angstroms) } \\
\hline & & & $\mathrm{X}$ & Y & Z \\
\hline & & & & & \\
\hline 1 & 15 & $\odot$ & - ๑. . ๑૦०००० & -1.153005 & $-\odot .000051$ \\
\hline 2 & 7 & $\odot$ & 1.188260 & ๑.067531 & -0.000055 \\
\hline 3 & 6 & 0 & ๑. 689097 & 1.338461 & $-\odot .000067$ \\
\hline 4 & 6 & ○ & -0.689097 & 1.338461 & $\odot .0 \odot \odot \odot 7 \odot$ \\
\hline 5 & 7 & $\odot$ & -1.188259 & $\odot .067531$ & $\odot .0 \odot \odot \odot 74$ \\
\hline 6 & 6 & ○ & -2.642803 & -0.204520 & -0.000011 \\
\hline 7 & 6 & 0 & 2.642803 & -0.204520 & $\odot .0 \odot \odot \odot 77$ \\
\hline 8 & 1 & 0 & 1.347775 & 2.193766 & -0.000098 \\
\hline 9 & 1 & $\odot$ & -1.347774 & 2.193766 & $\odot .000114$ \\
\hline 10 & 1 & $\odot$ & 2.800798 & -1.279121 & -0.000753 \\
\hline 11 & 1 & $\odot$ & 3.082468 & 0.228964 & -0.893564 \\
\hline 12 & 1 & $\odot$ & 3.082111 & $\odot .227570$ & $\odot .894572$ \\
\hline 13 & 1 & $\odot$ & -2.800797 & -1.279121 & $\odot .000637$ \\
\hline 14 & 1 & $\odot$ & -3.082426 & 0.228811 & $\odot .893725$ \\
\hline 15 & 1 & $\odot$ & -3.082153 & 0.227723 & -0.894411 \\
\hline
\end{tabular}

$$
\begin{aligned}
& \text { SCF Done: } E(R H F)=-605.553221462 \text { A.U. after } 14 \text { cycles } \\
& \text { Convg }=0.2706 \mathrm{D}-08 \quad-\mathrm{V} / \mathrm{T}=2.0017
\end{aligned}
$$

Spin components of $\mathrm{T}(2)$ and $\mathrm{E}(2)$ :
alpha-alpha $\mathrm{T} 2=$
$0.4787662857 \mathrm{D}-01 \mathrm{E} 2=$
alpha-beta T2 =
$0.2688734631 \mathrm{D}+00 \mathrm{E} 2=$
$-\odot .1282596661 D+0 \odot$
beta-beta T2 =
$0.4787662857 \mathrm{D}-01 \mathrm{E} 2=$
$-\odot .7568749518 D+0 \odot$
$-\odot .1282596661 D+\odot \odot$

ANorm $=0.1168172385 \mathrm{D}+01$

$\mathrm{E} 2=-\odot .1013394284 \mathrm{D}+01 \mathrm{EUMP} 2=-\odot .60656661574558 \mathrm{D}+03$ 


\section{Bis-(Dimethylamino)-phosphenium cation 3b:}

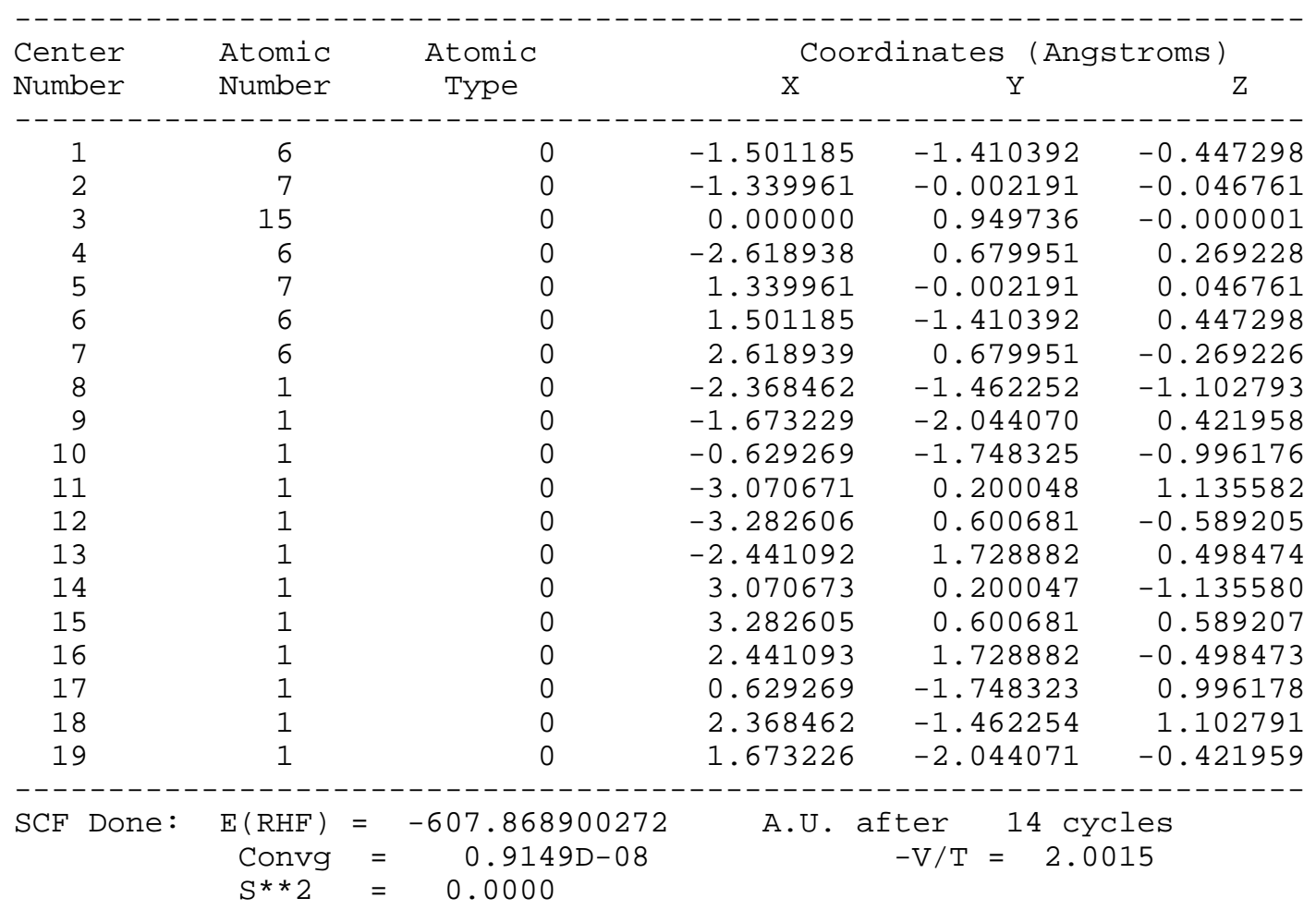

Spin components of $T(2)$ and $E(2)$ :
alpha-alpha T2 =
$0.4315022835 \mathrm{D}-01 \mathrm{E} 2=$
alpha-beta T2 =
$\odot .2667136246 \mathrm{D}+0 \odot \mathrm{E} 2=$
beta-beta T2 =
$0.4315022835 \mathrm{D}-01 \mathrm{E} 2=$
$-\odot .1229188655 D+\odot \odot$
$-\odot .7985824785 D+\odot \odot$
$-\odot .1229188655 \mathrm{D}+\odot \odot$

ANorm $=\quad 0.1163191335 \mathrm{D}+01$

$\mathrm{E} 2=-0.1044420209 \mathrm{D}+01$ EUMP2 $=-0.60891332048092 \mathrm{D}+03$

\section{2-Fluoro-1,3-Dimethyl-1,3,2-diazaphospholene 1b[F]:}

\begin{tabular}{|c|c|c|c|c|c|}
\hline \multirow{2}{*}{$\begin{array}{l}\text { Center } \\
\text { Number }\end{array}$} & \multirow{2}{*}{$\begin{array}{l}\text { Atomic } \\
\text { Number }\end{array}$} & \multirow{2}{*}{$\begin{array}{l}\text { Atomic } \\
\text { Type }\end{array}$} & \multicolumn{3}{|c|}{ Coordinates (Angstroms) } \\
\hline & & & $\mathrm{X}$ & $\mathrm{Y}$ & Z \\
\hline 1 & 9 & 0 & $-ค ค ค ค ค 162$ & $-1674 \rho \cap 5$ & ๑ 989987 \\
\hline$\frac{1}{2}$ & 15 & $\odot$ & $\begin{array}{l}-0.000162 \\
-0.000004\end{array}$ & $\begin{array}{l}-1.6 / 4005 \\
-0.848407\end{array}$ & $\begin{array}{r}0.989981 \\
-0.504632\end{array}$ \\
\hline 3 & 7 & $\odot$ & -1.187779 & 0.348543 & -0.249995 \\
\hline 4 & 6 & $\odot$ & -2.613409 & $\odot .043980$ & $-\odot .20 \odot 969$ \\
\hline 5 & 7 & $\odot$ & 1.187832 & 0.348446 & -0.249889 \\
\hline 6 & 6 & $\odot$ & 2.613460 & 0.043872 & -0.200956 \\
\hline 7 & 6 & 0 & $\odot .677418$ & 1.503060 & ๑. 363251 \\
\hline 8 & 6 & $\odot$ & -0.677324 & 1.503097 & 0.363221 \\
\hline 9 & 1 & 0 & 1.341314 & 2.278049 & 0.712337 \\
\hline 10 & 1 & $\odot$ & -1.341191 & 2.278124 & $\odot .712275$ \\
\hline 11 & 1 & $\odot$ & 2.821970 & -0.789786 & $-\odot .869352$ \\
\hline 12 & 1 & $\odot$ & 3. 178902 & ๑. 909354 & -0.544895 \\
\hline 13 & 1 & $\odot$ & 2.934693 & -0.223247 & $\odot .807678$ \\
\hline 14 & 1 & $\odot$ & -2.822005 & -0.789555 & $-\odot .869492$ \\
\hline 15 & 1 & $\odot$ & -2.934542 & -0.223306 & $\odot .807652$ \\
\hline 16 & 1 & $\odot$ & -3.178866 & $\odot .909534$ & -0.544700 \\
\hline
\end{tabular}

\footnotetext{
SCF Done: $E($ RHF $)=-705.221857632$ A.U. after 8 cycles

$$
\text { Convg }=0.5266 \mathrm{D}-\odot 8 \quad-\mathrm{V} / \mathrm{T}=2.0019
$$$$
\mathrm{S}^{* * 2}=0.0000
$$ 
Spin components of $T(2)$ and $E(2)$ :
alpha-alpha T2 =
$0.5110034796 \mathrm{D}-01 \mathrm{E} 2=$
$-\odot .1532613808 D+\odot \odot$
alpha-beta T2 =
$\odot .2878751349 D+0 \odot$ E2=
$-\odot .8890 \odot 26026 D+\odot \odot$
$-\odot .1532613808 D+\odot \odot$ beta-beta $\mathrm{T} 2=$

ANorm $=\quad 0.1179014771 \mathrm{D}+01$

$\mathrm{E} 2=-\odot .1195525364 \mathrm{D}+01$ EUMP2 $=-\odot .70641738299586 \mathrm{D}+\odot 3$

2-Chloro-1,3-Dimethyl-1,3,2-diazaphospholene 1b[Cl]:

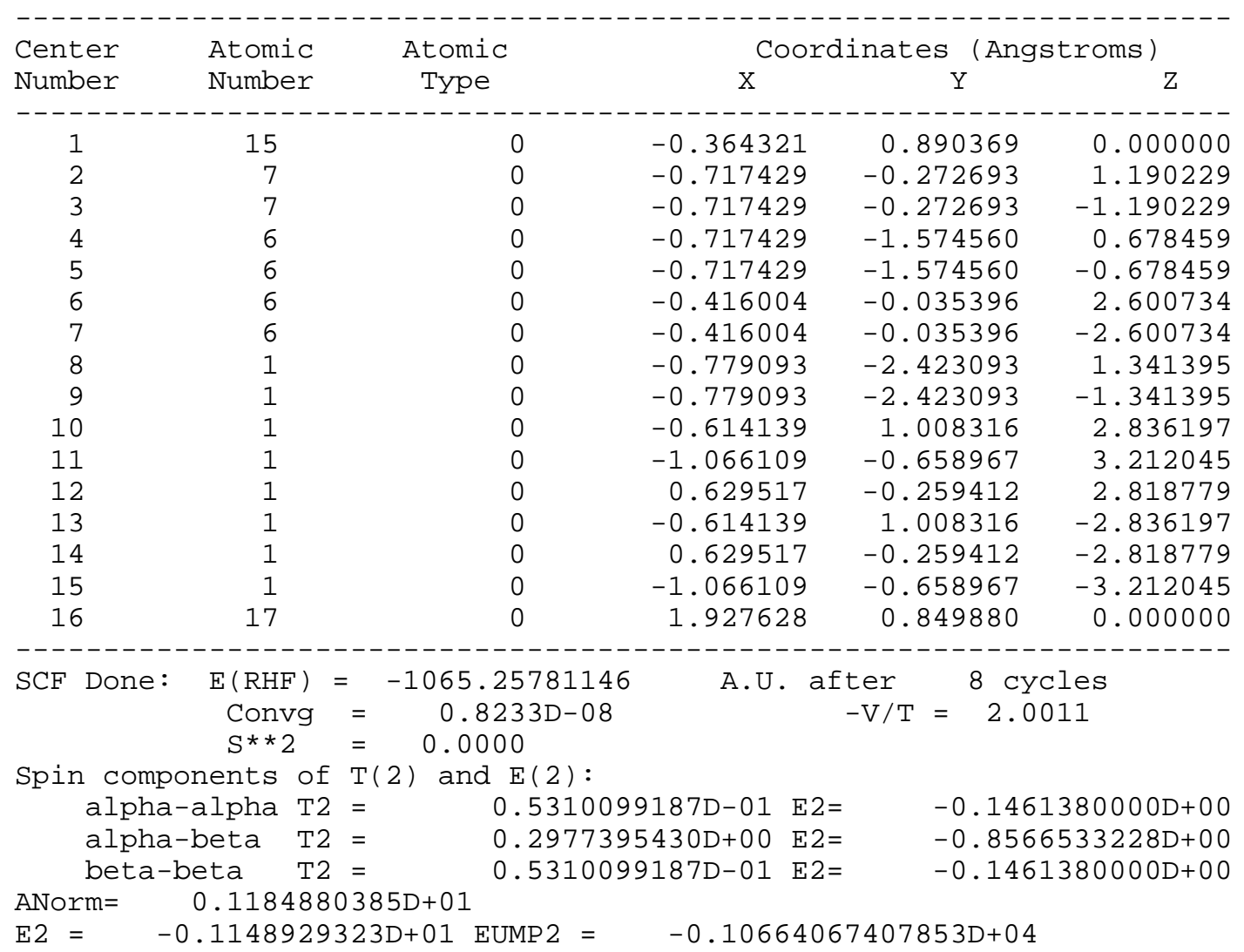

Chloro-bis-(dimethylamino)-phosphine $3 \mathrm{~b}[\mathrm{Cl}]$ :

\begin{tabular}{|c|c|c|c|c|c|}
\hline \multirow{2}{*}{$\begin{array}{l}\text { Center } \\
\text { Number }\end{array}$} & Atomic & Atomic & \multicolumn{3}{|c|}{ Coordinates (Angstroms) } \\
\hline & Number & Type & $\mathrm{X}$ & $\mathrm{Y}$ & Z \\
\hline & & $-\ldots--$ & ------ & ------6 & ------- \\
\hline 1 & 6 & $\odot$ & 1.755357 & -0.364199 & 1.299246 \\
\hline 2 & 7 & ○ & 1.360759 & -0.560555 & -0.096977 \\
\hline 3 & 15 & $\odot$ & -0.015483 & 0.129389 & -0.816638 \\
\hline 4 & 17 & $\odot$ & -0.241253 & 1.945798 & $\odot .379281$ \\
\hline 5 & 6 & $\odot$ & 2.534237 & -0.601417 & -0.977247 \\
\hline 6 & 7 & $\odot$ & -1.279131 & -0.838108 & -0.229313 \\
\hline 7 & 6 & $\odot$ & -1.286816 & -1.466519 & 1.086650 \\
\hline 8 & 6 & $\odot$ & -2.605137 & -0.359049 & $-\odot .627983$ \\
\hline 9 & 1 & 0 & 2.380555 & 0.528204 & 1.402872 \\
\hline 10 & 1 & 0 & 2.324347 & -1.235071 & 1.631518 \\
\hline 11 & 1 & 0 & ๑.889335 & -0.241629 & 1.939689 \\
\hline 12 & 1 & $\odot$ & 3.158814 & -1.448658 & -0.689950 \\
\hline 13 & 1 & 0 & 3.133150 & 0.313516 & -0.901847 \\
\hline 14 & 1 & 0 & 2.223075 & -0.739386 & -2.010352 \\
\hline 15 & 1 & $\odot$ & -2.998670 & ๑. 382301 & $\odot .075658$ \\
\hline 16 & 1 & $\odot$ & -3.289162 & -1.207143 & -0.672757 \\
\hline
\end{tabular}




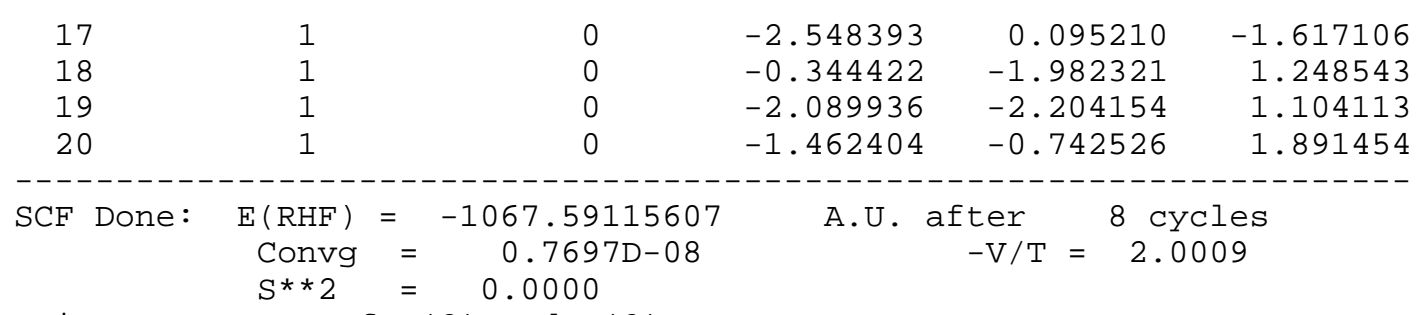

Spin components of $T(2)$ and $E(2)$ :

alpha-alpha T2 $=\quad 0.5054307308 \mathrm{D}-01 \mathrm{E} 2=$

alpha-beta T2 $=0.2999230725 \mathrm{D}+00 \mathrm{E} 2=$

beta-beta $\mathrm{T} 2=0.5054307308 \mathrm{D}-01 \mathrm{E} 2=$

$-\odot .1435626863 D+0 \odot$

$-\odot .9017639384 D+\odot \odot$

$-\odot .1435626863 D+\odot \odot$

ANorm $=\quad 0.1183642353 \mathrm{D}+01$

$\mathrm{E} 2=-\Theta .1188889311 \mathrm{D}+01$ EUMP2 $=-\Theta .10687800453762 \mathrm{D}+\odot 4$

F-bridged 2-Fluoro-1,3-Dimethyl-1,3,2-diazaphospholene / 1,3-Dimethyl-1,3,2-

\section{diazaphospholenium Complex 4'b[F]:}

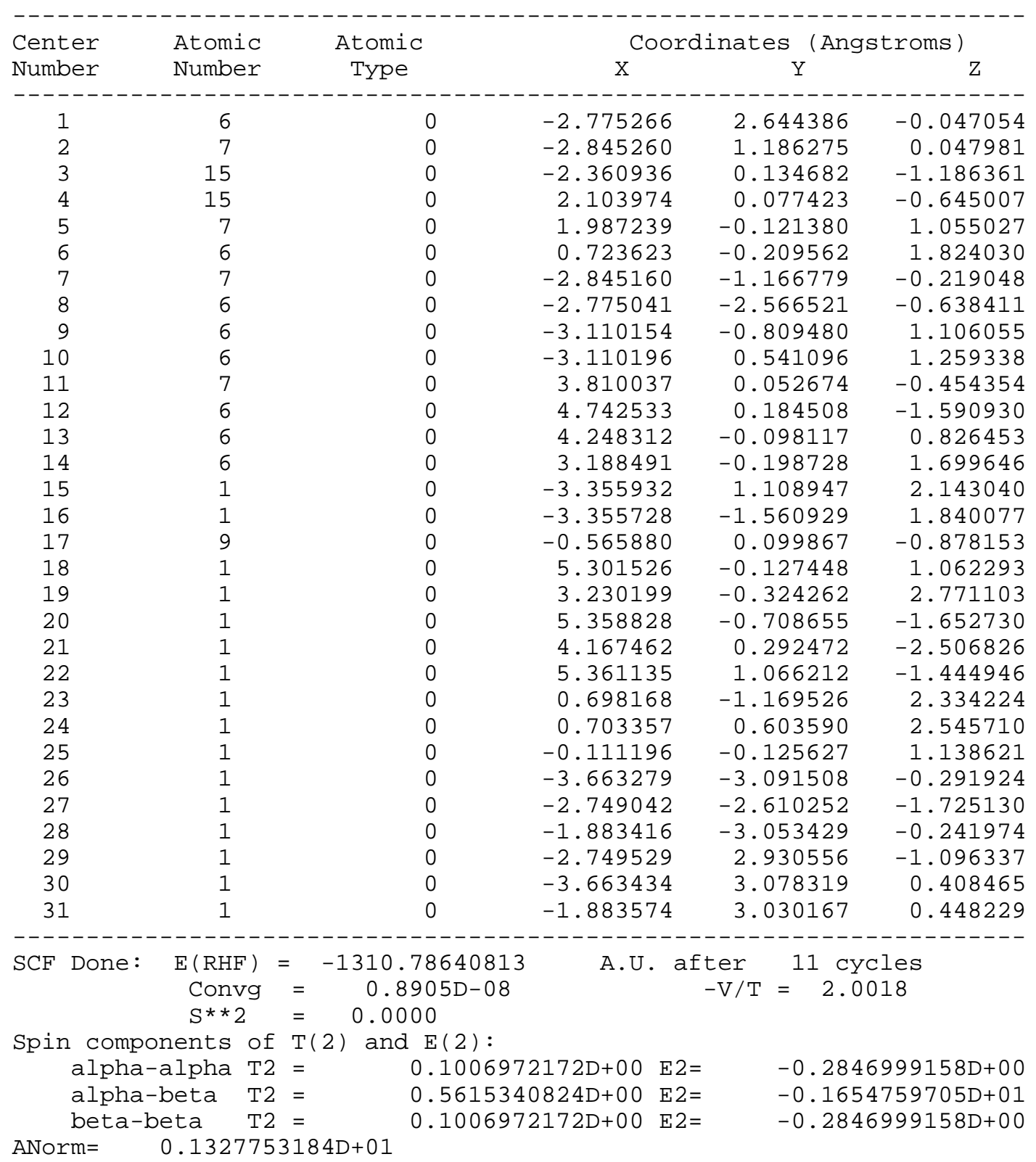


F-bridged 2-Fluoro-1,3-Dimethyl-1,3,2-diazaphospholene / 1,3-Dimethyl-1,3,2-

diazaphospholenium Complex $4 \mathrm{~b}[\mathrm{~F}]\left(\mathrm{C}_{2}\right.$-symmetric Transition State):

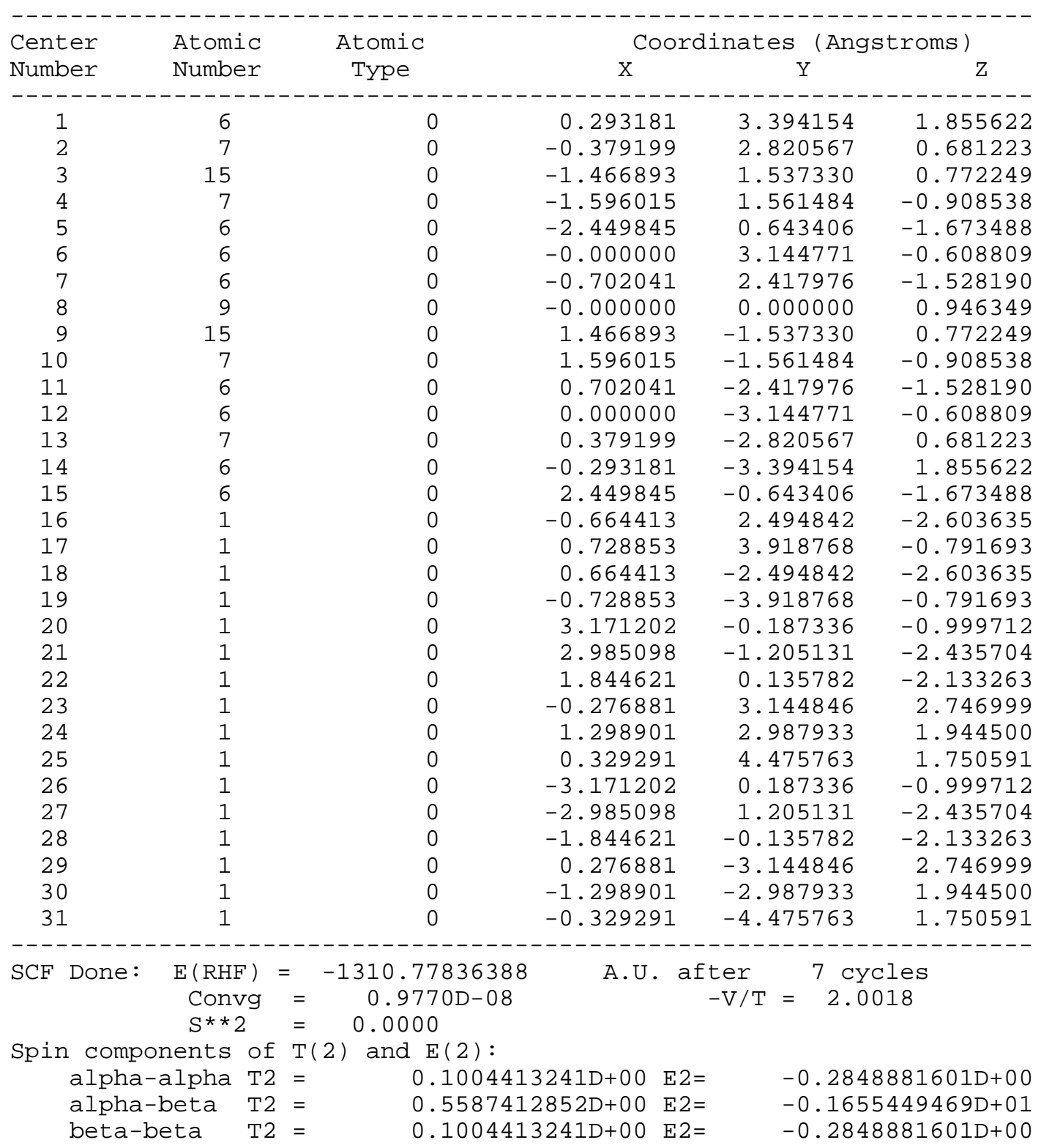

ANorm $=\quad 0.1326508173 \mathrm{D}+01$

$\mathrm{E} 2=-\odot .2225225789 \mathrm{D}+01$ EUMP2 $=-\odot .13130035896697 \mathrm{D}+\odot 4$

Cl-bridged 2-Chloro-1,3-Dimethyl-1,3,2-diazaphospholene / 1,3-Dimethyl-1,3,2-

diazaphospholenium Complex 4b[Cl]:

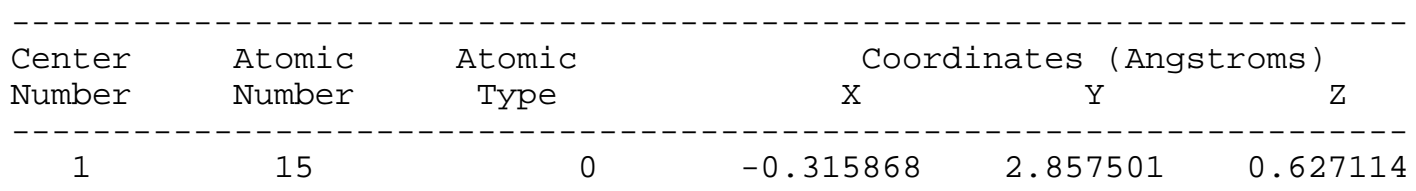




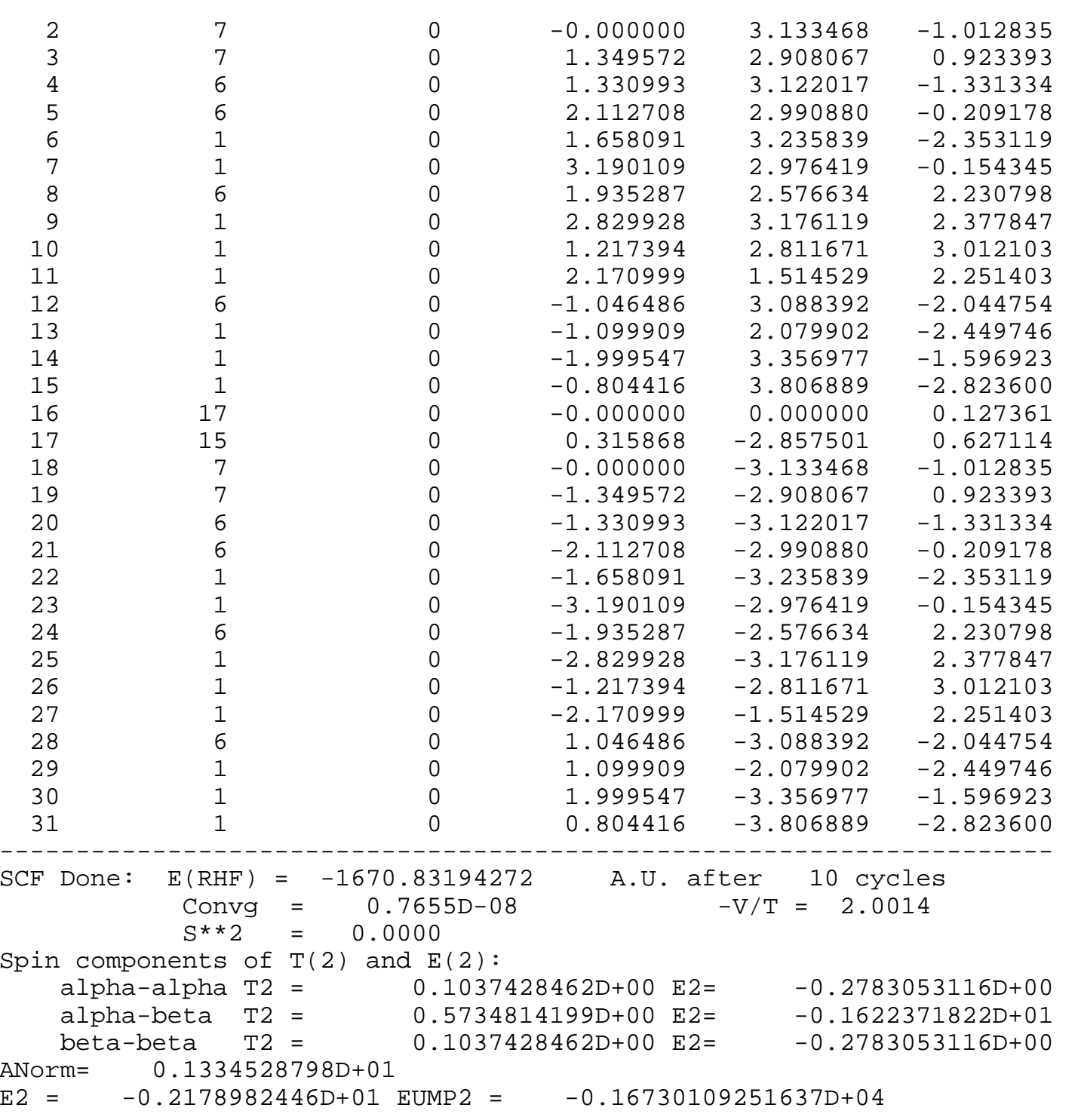

Cl-bridged Chloro-bis(dimethylamino)-phosphine / bis(dimethylamino)-phosphenium Complex

$6 \mathrm{~b}[\mathrm{Cl}]$ :

\begin{tabular}{|c|c|c|c|c|c|}
\hline \multirow{2}{*}{$\begin{array}{l}\text { Center } \\
\text { Number }\end{array}$} & \multirow{2}{*}{$\begin{array}{l}\text { Atomic } \\
\text { Number }\end{array}$} & \multirow{2}{*}{$\begin{array}{c}\text { Atomic } \\
\text { Type }\end{array}$} & \multicolumn{3}{|c|}{ Coordinates (Angstroms) } \\
\hline & & & $\mathrm{x}$ & $\mathrm{Y}$ & z \\
\hline 1 & 6 & $\odot$ & -2.618636 & 2.123266 & -0.373820 \\
\hline 2 & 6 & 0 & -2.448807 & 1.857291 & 2.058446 \\
\hline 3 & 7 & 0 & -2.631536 & 1.188319 & 0.758828 \\
\hline 4 & 6 & 0 & -2.711578 & -2.428981 & -0.932023 \\
\hline 5 & 6 & 0 & -4.035041 & -0.418461 & -1.465843 \\
\hline 6 & 7 & 0 & -3.121509 & -1.074487 & -0.529380 \\
\hline 7 & 15 & 0 & -2.271211 & -0.424737 & 0.727265 \\
\hline 8 & 15 & 0 & 2.232628 & 0.432704 & 0.717523 \\
\hline 9 & 7 & 0 & 3.014412 & 1.055078 & -0.597117 \\
\hline 10 & 6 & $\Theta$ & 3.145998 & 0.446834 & -1.928668 \\
\hline 11 & 6 & $\Theta$ & 3.161422 & 2.521623 & -0.617585 \\
\hline 12 & 7 & 0 & 2.729001 & -1.133892 & 0.877635 \\
\hline 13 & 6 & $\Theta$ & 1.910592 & -1.957179 & 1.779844 \\
\hline 14 & 6 & 0 & 3.663195 & -1.898584 & 0.051014 \\
\hline
\end{tabular}




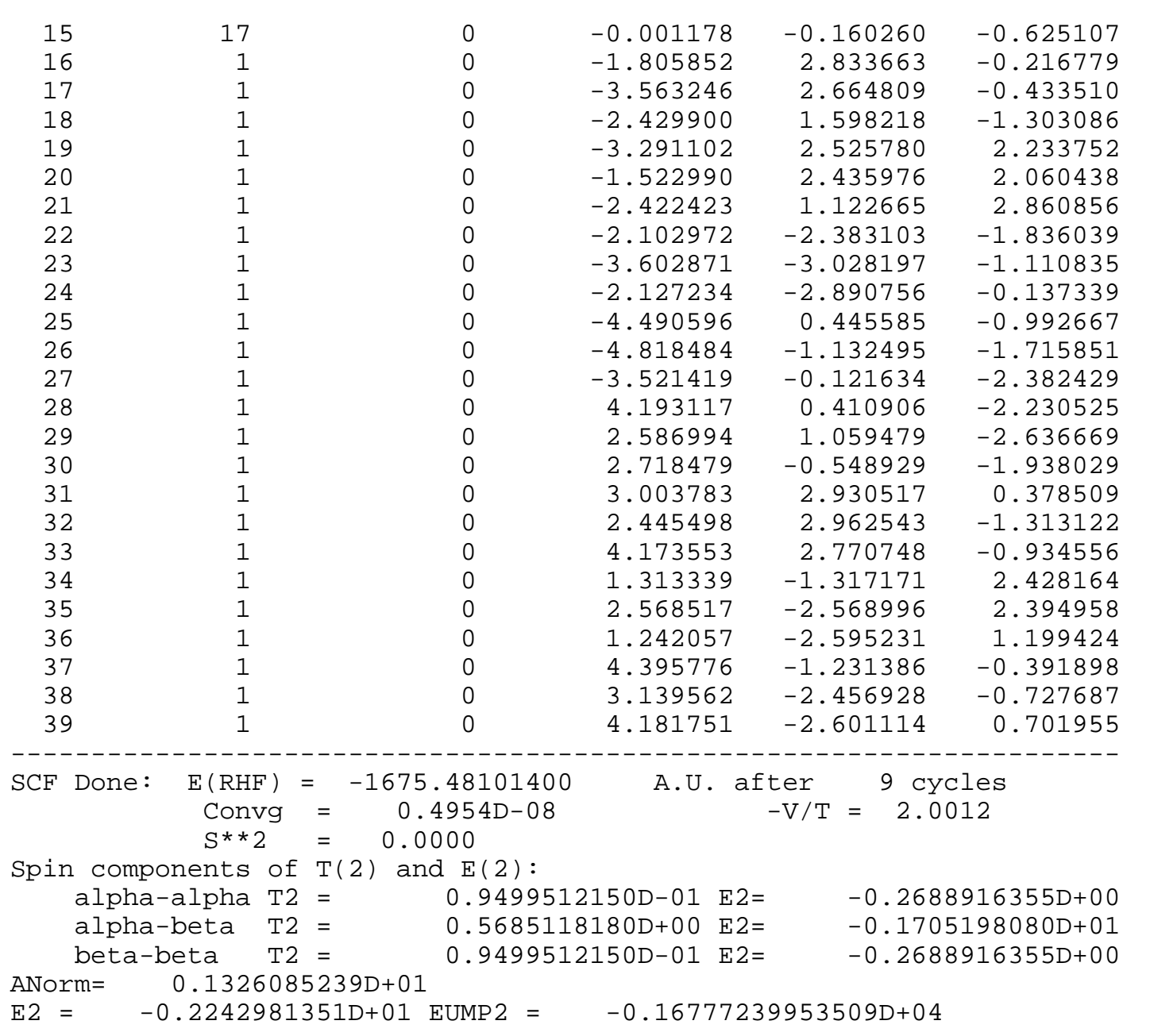

\section{P-P bonded Chloro-bis(dimethylamino)-phosphine / bis(dimethylamino)-phosphenium Complex}

\section{6'b[Cl]:}

\begin{tabular}{|c|c|c|c|c|c|}
\hline \multirow{2}{*}{$\begin{array}{l}\text { Center } \\
\text { Number }\end{array}$} & \multirow{2}{*}{$\begin{array}{l}\text { Atomic } \\
\text { Number }\end{array}$} & \multirow{2}{*}{$\begin{array}{l}\text { Atomic } \\
\text { Type }\end{array}$} & \multicolumn{3}{|c|}{ Coordinates (Angstroms) } \\
\hline & & & $x$ & $\mathrm{Y}$ & Z \\
\hline & & & $-8-2-6$ & & $\cdots-\cdots$ \\
\hline 1 & 7 & 0 & -2.218613 & -0.965879 & -0.755796 \\
\hline 2 & 15 & $\odot$ & -1.070254 & $\odot . \odot 45703$ & -0.104438 \\
\hline 3 & 15 & $\odot$ & 1.830539 & -0.233089 & -1.103696 \\
\hline 4 & 7 & $\odot$ & 2.228169 & -1.325634 & 0.063707 \\
\hline 5 & 17 & 0 & -0.609321 & -0.707338 & 1.847427 \\
\hline 6 & 7 & 0 & -2.070418 & 1.356530 & $\odot .361255$ \\
\hline 7 & 7 & 0 & 2. 282397 & 1.258600 & -0.560336 \\
\hline 8 & 6 & 0 & -3.485650 & -1.263654 & -0.073461 \\
\hline 9 & 6 & ○ & -1.790161 & -2.015941 & -1.682485 \\
\hline 10 & 6 & $\odot$ & -1.524519 & 2.366723 & 1.274155 \\
\hline 11 & 6 & $\odot$ & -2.798160 & 1.993158 & -0.751892 \\
\hline 12 & 6 & $\odot$ & 2.223852 & 1.798469 & 0.805182 \\
\hline 13 & 6 & ○ & 1.683730 & -2.685050 & -0.115112 \\
\hline 14 & 6 & 0 & 3. 025107 & -1.159362 & 1.284928 \\
\hline 15 & 6 & 0 & 2.443852 & 2.297492 & -1.596425 \\
\hline 16 & 1 & $\odot$ & 2.381498 & -1.072289 & 2.160810 \\
\hline 17 & 1 & $\odot$ & 3.650702 & -2.044866 & 1.386651 \\
\hline 18 & 1 & $\odot$ & 3.668819 & -0.290793 & 1.193701 \\
\hline 19 & 1 & $\odot$ & 0.975764 & -2.900571 & 0.684416 \\
\hline
\end{tabular}




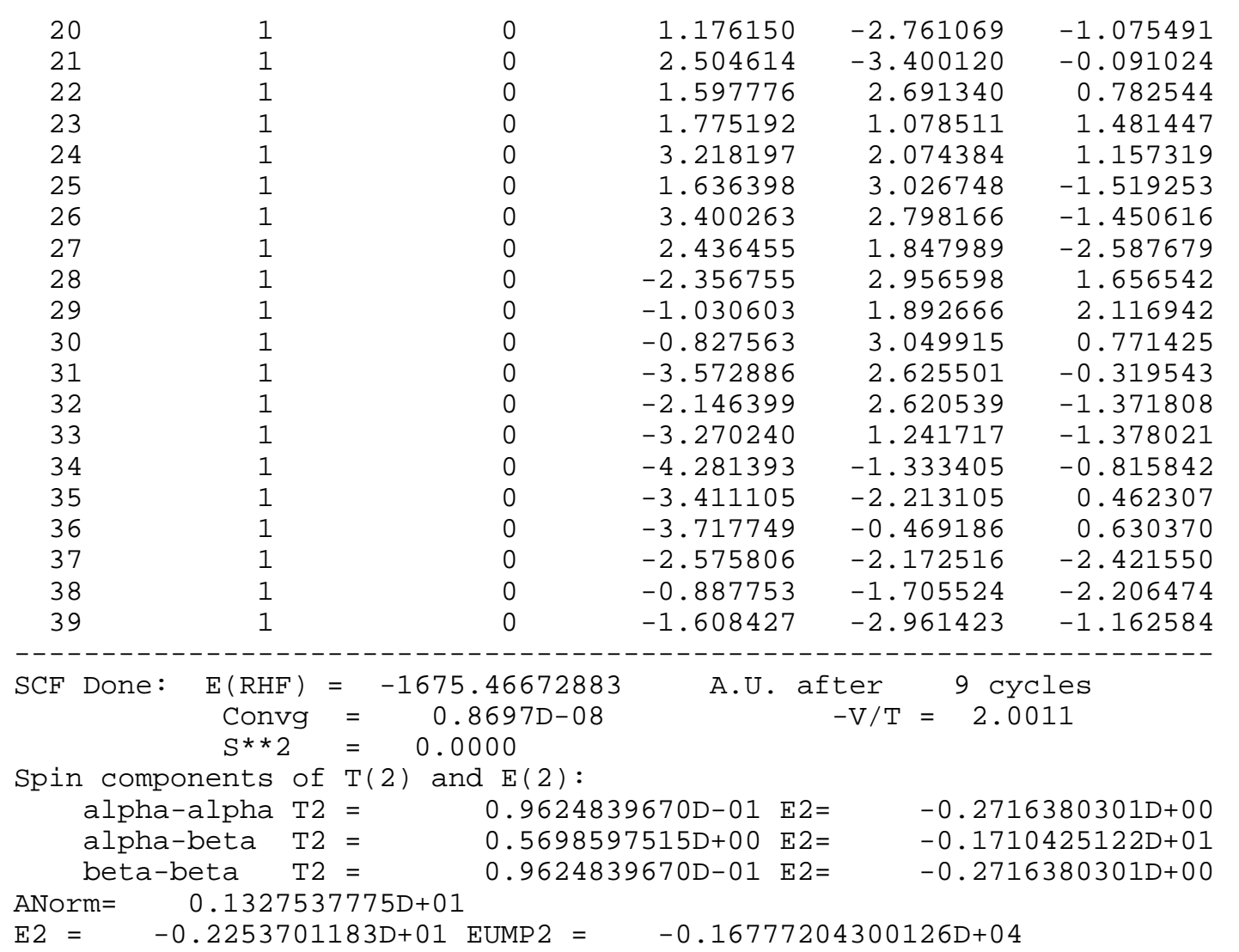

Final atomic coordinates and energies for species in $\mathrm{CH}_{2} \mathrm{Cl}_{2}$ solution at b3lyp/6-31+g** level:

\section{1,3-Dimethyl-1,3,2-diazaphospholenium cation 1b:}

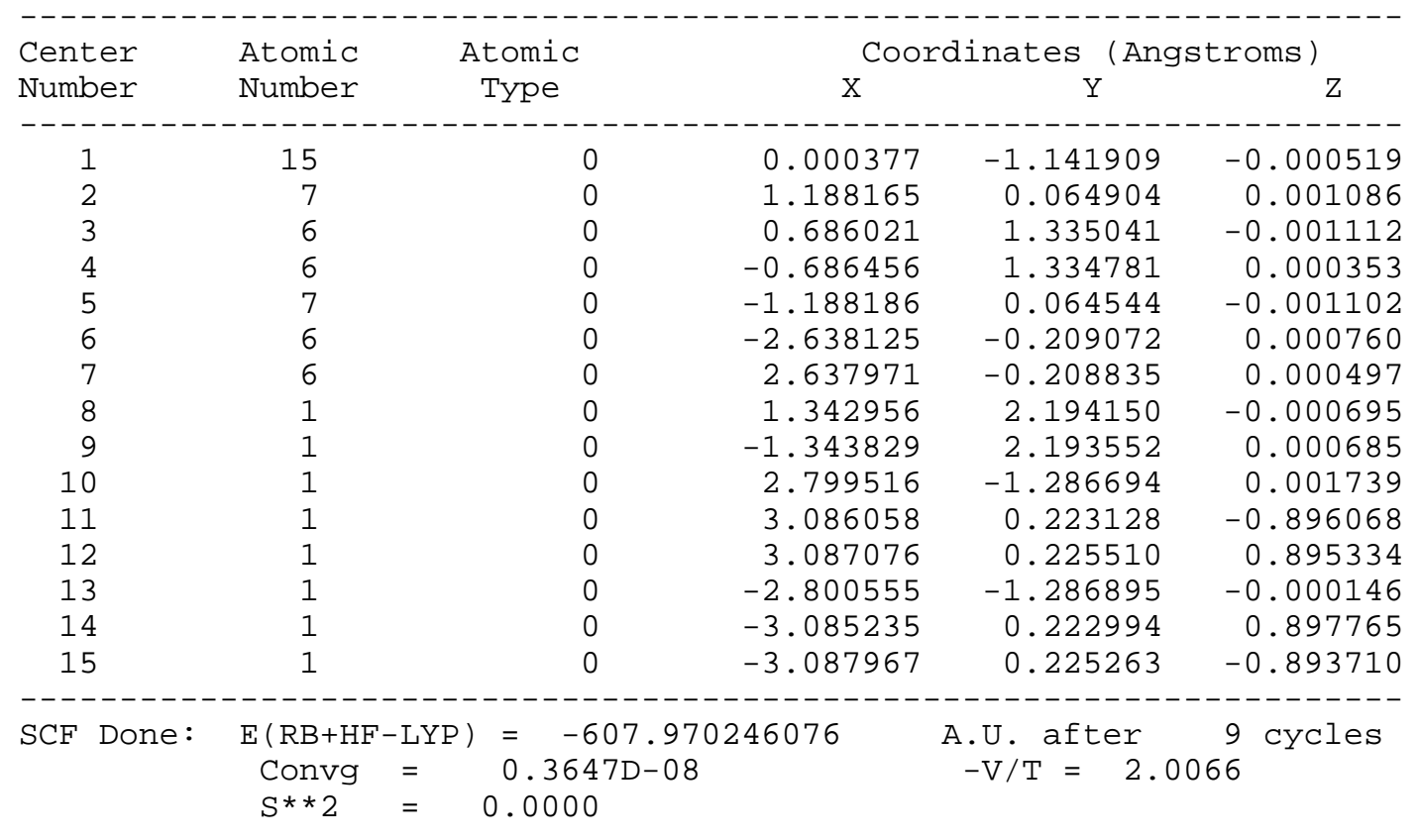

Variational C-PCM results

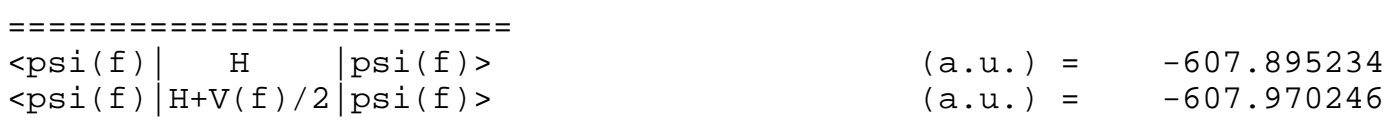


Total free energy in solution:

with all non electrostatic terms $($ a.u. $)=-607.966642$

(Polarized solute)-Solvent

$(\mathrm{kcal} / \mathrm{mol})=-47.07$

Cavitation energy

Dispersion energy

Repulsion energy

Total non electrostatic

(kcal/mol) $=2014.25$

$(\mathrm{kcal} / \mathrm{mol})=14.25$

$(\mathrm{kcal} / \mathrm{mol})=-13.28$

$(\mathrm{kcal} / \mathrm{mol})=1.29$

$(\mathrm{kcal} / \mathrm{mol})=2.26$

\section{1,3-Dimethyl-1,3,2-diazaphospholidinium cation 2b:}

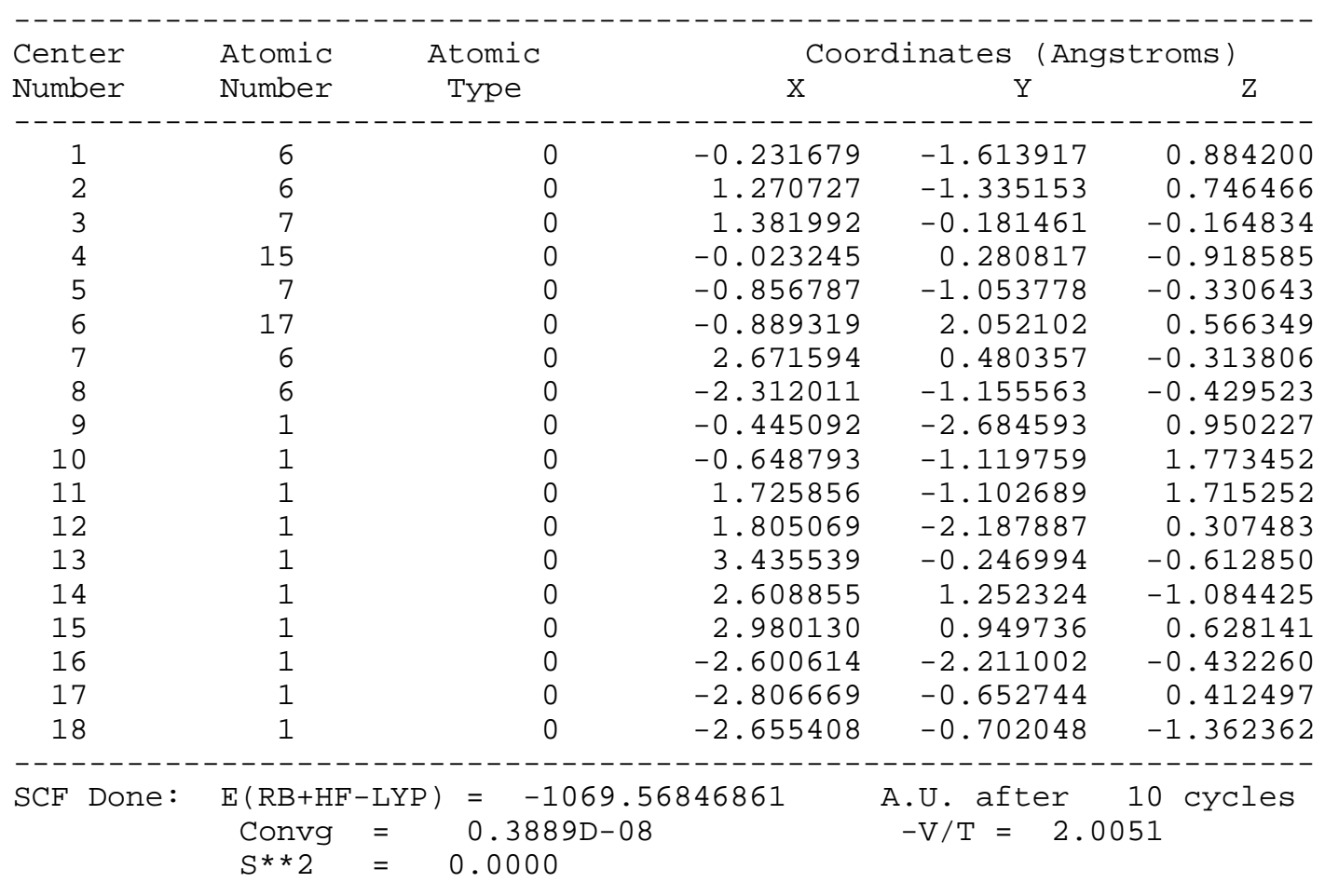

Variational C-PCM results

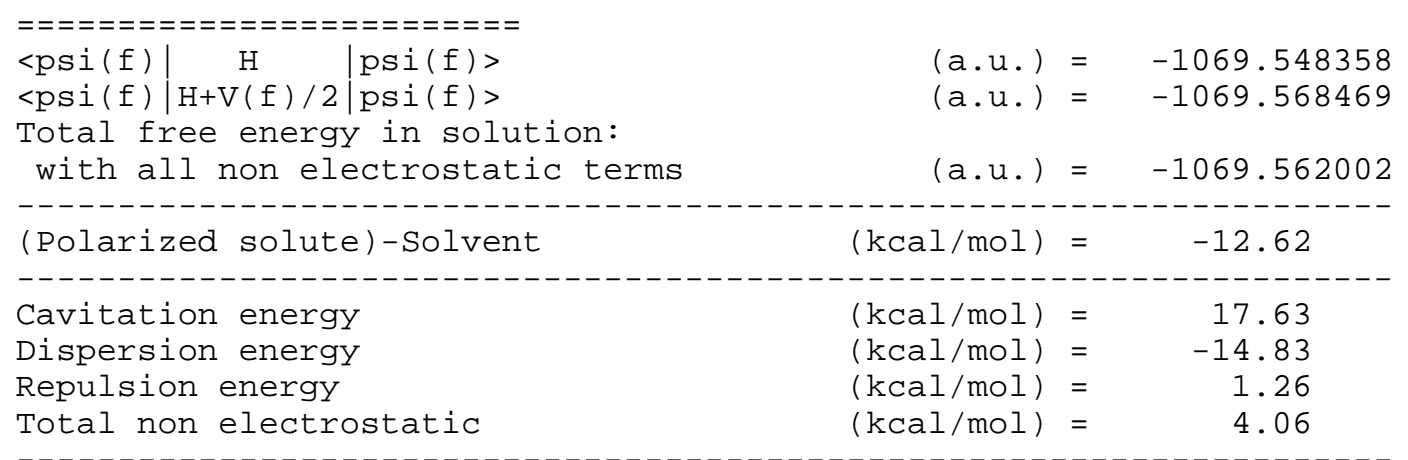

Bis-(Dimethylamino)-phosphenium cation 3b:

$\begin{array}{llll}\text { Center } & \text { Atomic } & \text { Atomic } & \text { Coordinates (Angstroms) } \\ \text { Number } & \text { Number } & \text { Type } & \mathrm{X}\end{array}$




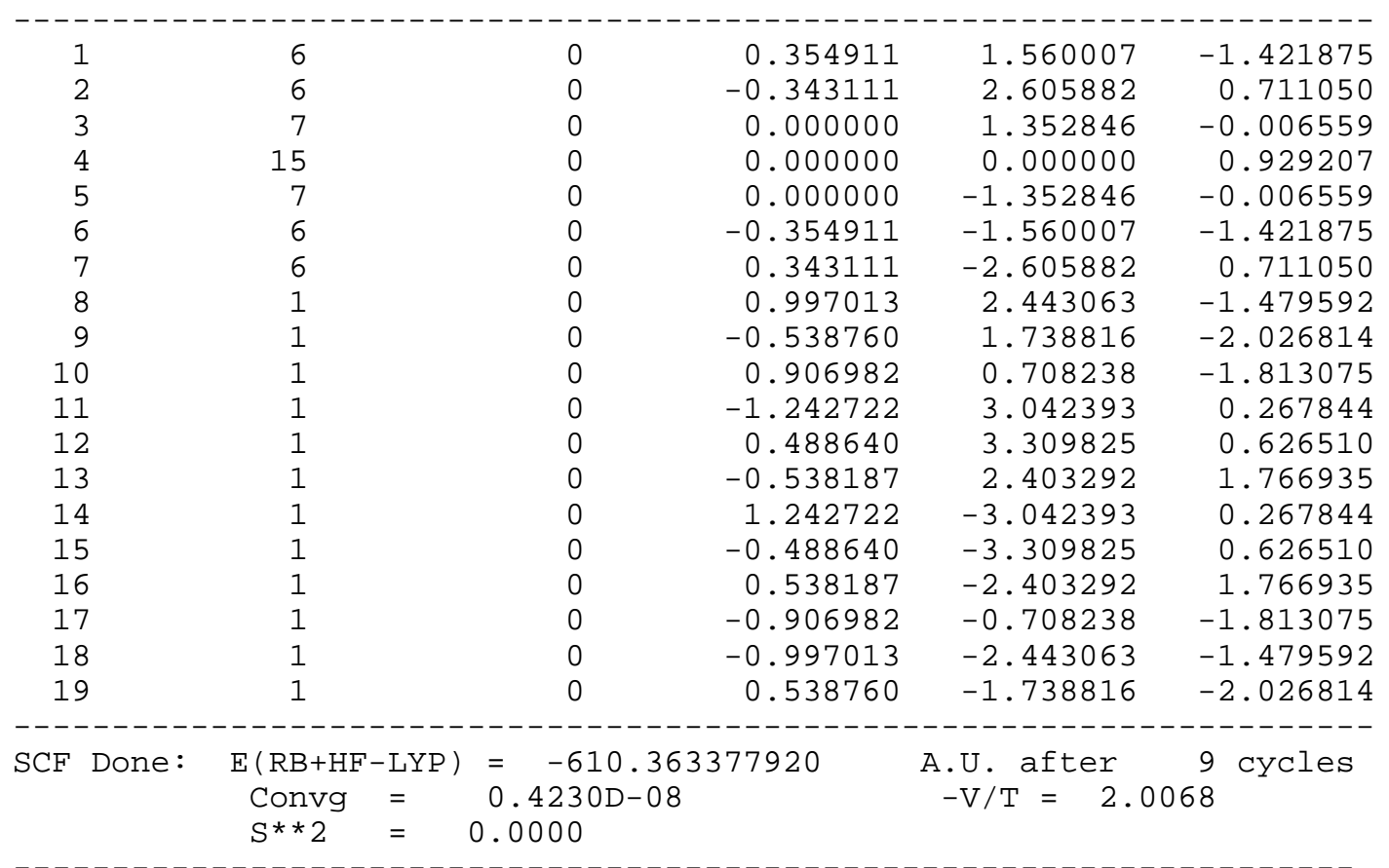

Variational C-PCM results

\begin{tabular}{|c|c|c|}
\hline $\begin{array}{l}<p s i(f) \mid H \\
<p s i(f)|H+V(f) / 2| \operatorname{psi}(f)> \\
\text { Total free energy in solution: } \\
\text { with all non electrostatic terms }\end{array}$ & $\begin{array}{l}(\text { a.u. })= \\
(\text { a.u. })= \\
(\text { a.u. })=\end{array}$ & $\begin{array}{l}-610.291251 \\
-610.363378 \\
-610.358104\end{array}$ \\
\hline (Polarized solute)-Solvent & $(\mathrm{kcal} / \mathrm{mol})$ & -45.26 \\
\hline $\begin{array}{l}\text { Cavitation energy } \\
\text { Dispersion energy } \\
\text { Repulsion energy } \\
\text { Total non electrostatic }\end{array}$ & $\begin{array}{l}(\mathrm{kcal} / \mathrm{mol})= \\
(\mathrm{kcal} / \mathrm{mol})= \\
(\mathrm{kcal} / \mathrm{mol})= \\
(\mathrm{kcal} / \mathrm{mol})=\end{array}$ & $\begin{array}{r}16.48 \\
-14.44 \\
1.27 \\
3.31\end{array}$ \\
\hline
\end{tabular}

2-Fluoro-1,3-Dimethyl-1,3,2-diazaphospholene 1b[F]:

\begin{tabular}{|c|c|c|c|c|c|}
\hline \multirow{2}{*}{$\begin{array}{l}\text { Center } \\
\text { Number }\end{array}$} & \multirow{2}{*}{$\begin{array}{l}\text { Atomic } \\
\text { Number }\end{array}$} & \multirow{2}{*}{$\begin{array}{l}\text { Atomic } \\
\text { Type }\end{array}$} & \multicolumn{3}{|c|}{ Coordinates (Angstroms) } \\
\hline & & & $x$ & $\mathrm{Y}$ & Z \\
\hline 1 & 9 & $\Theta$ & 0.001608 & -1.755928 & $\odot .999222$ \\
\hline 2 & 15 & $\odot$ & -0.000126 & -0.840453 & -0.501656 \\
\hline 3 & 7 & $\Theta$ & -1.193313 & 0.332644 & -0.185280 \\
\hline 4 & 6 & $\Theta$ & -2.632282 & 0.088804 & -0.280030 \\
\hline 5 & 7 & $\Theta$ & 1.192815 & 0.333311 & -0.185893 \\
\hline 6 & 6 & $\Theta$ & 2.631902 & 0.089917 & -0.280274 \\
\hline 7 & 6 & $\Theta$ & 0.674762 & 1.499050 & 0.403837 \\
\hline 8 & 6 & $\Theta$ & -0.675601 & 1.498734 & 0.404189 \\
\hline 9 & 1 & $\Theta$ & 1.340564 & 2.270717 & 0.764829 \\
\hline 10 & 1 & $\Theta$ & -1.341448 & 2.270222 & 0.765619 \\
\hline 11 & 1 & $\Theta$ & 2.812430 & -0.773525 & -0.924822 \\
\hline 12 & 1 & $\odot$ & 3.127255 & 0.959377 & -0.722698 \\
\hline 13 & 1 & $\odot$ & 3.071133 & -0.109894 & 0.703734 \\
\hline 14 & 1 & $\odot$ & -2.812215 & -0.776116 & -0.922603 \\
\hline 15 & 1 & $\odot$ & -3.072010 & -0.108641 & $\odot .704171$ \\
\hline 16 & 1 & $\Theta$ & -3.127495 & 0.957289 & -0.724525 \\
\hline
\end{tabular}




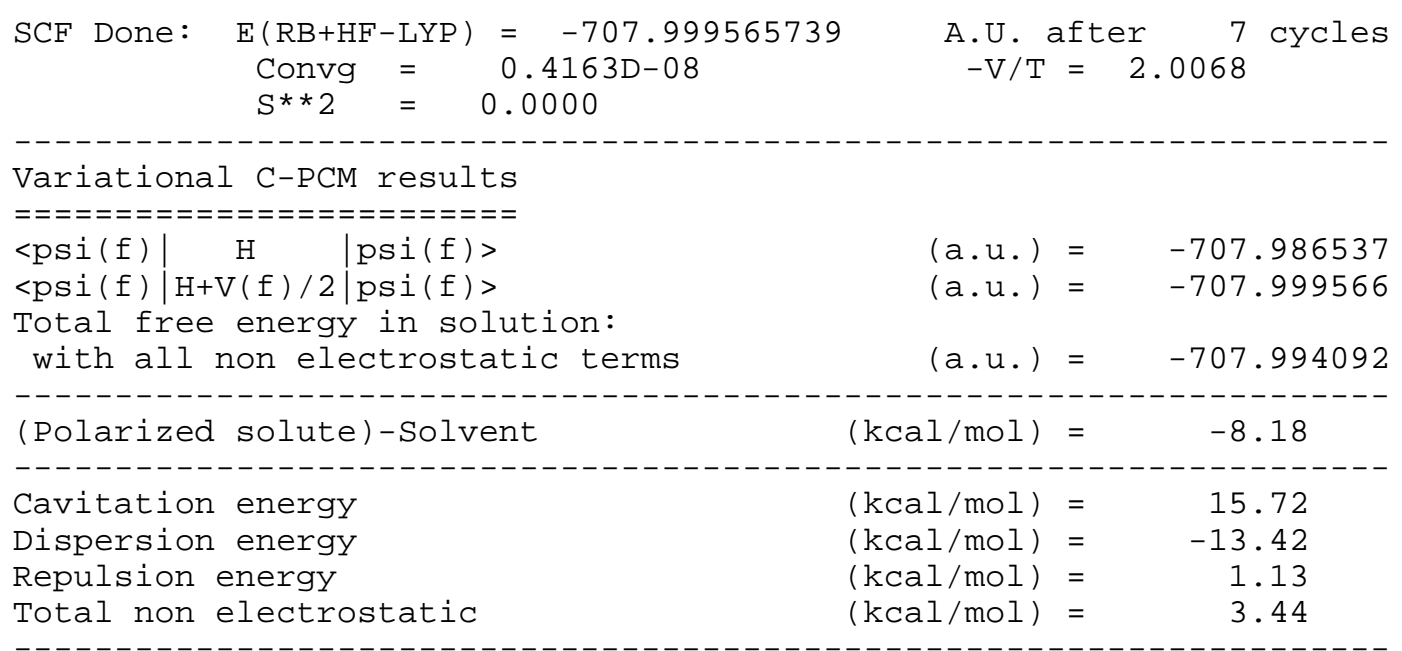

\section{2-Chloro-1,3-Dimethyl-1,3,2-diazaphospholene $1 \mathrm{~b}[\mathrm{Cl}]$ :}

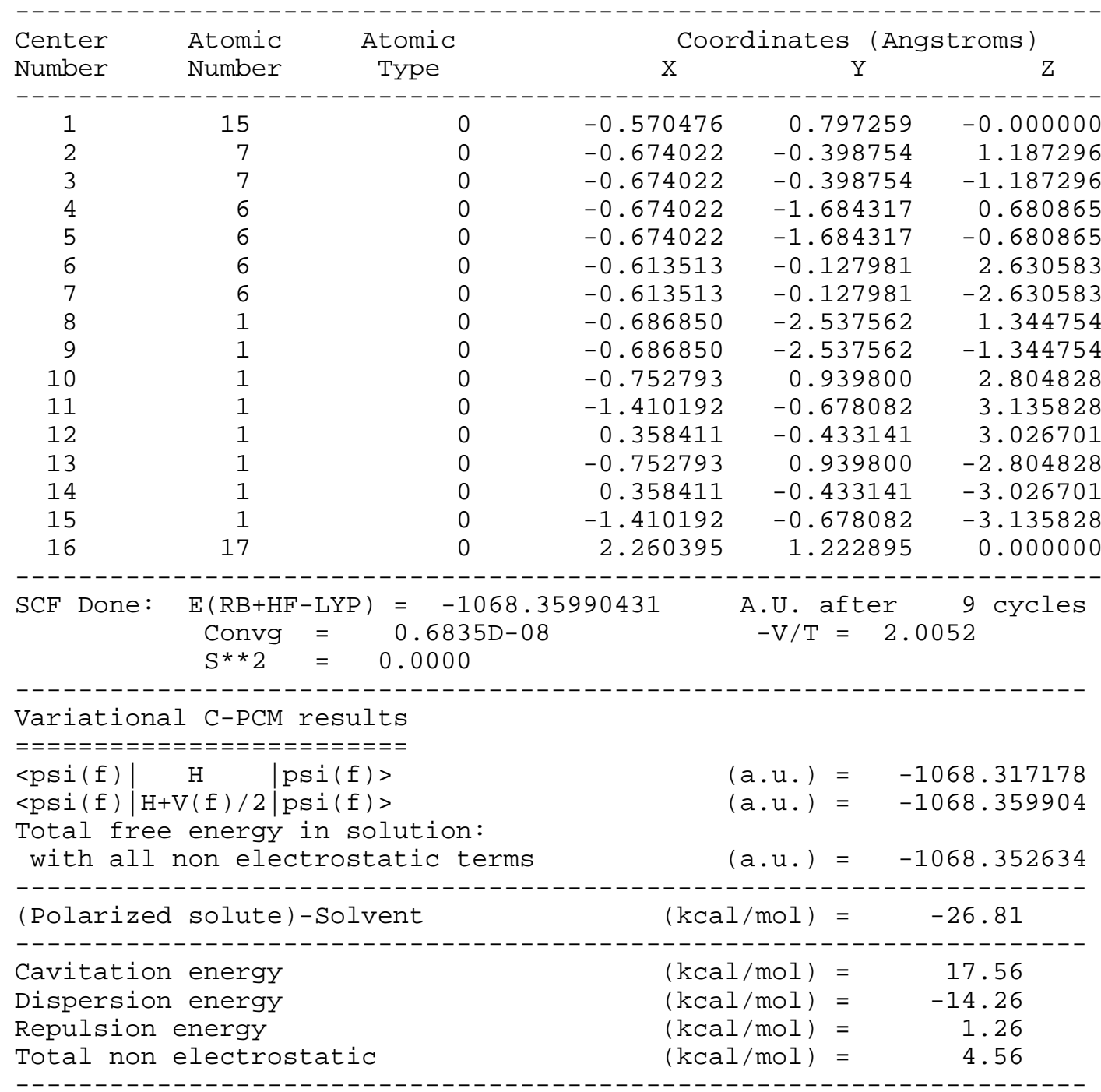




\section{2-Bromo-1,3-Dimethyl-1,3,2-diazaphospholene 1b[Br]:}

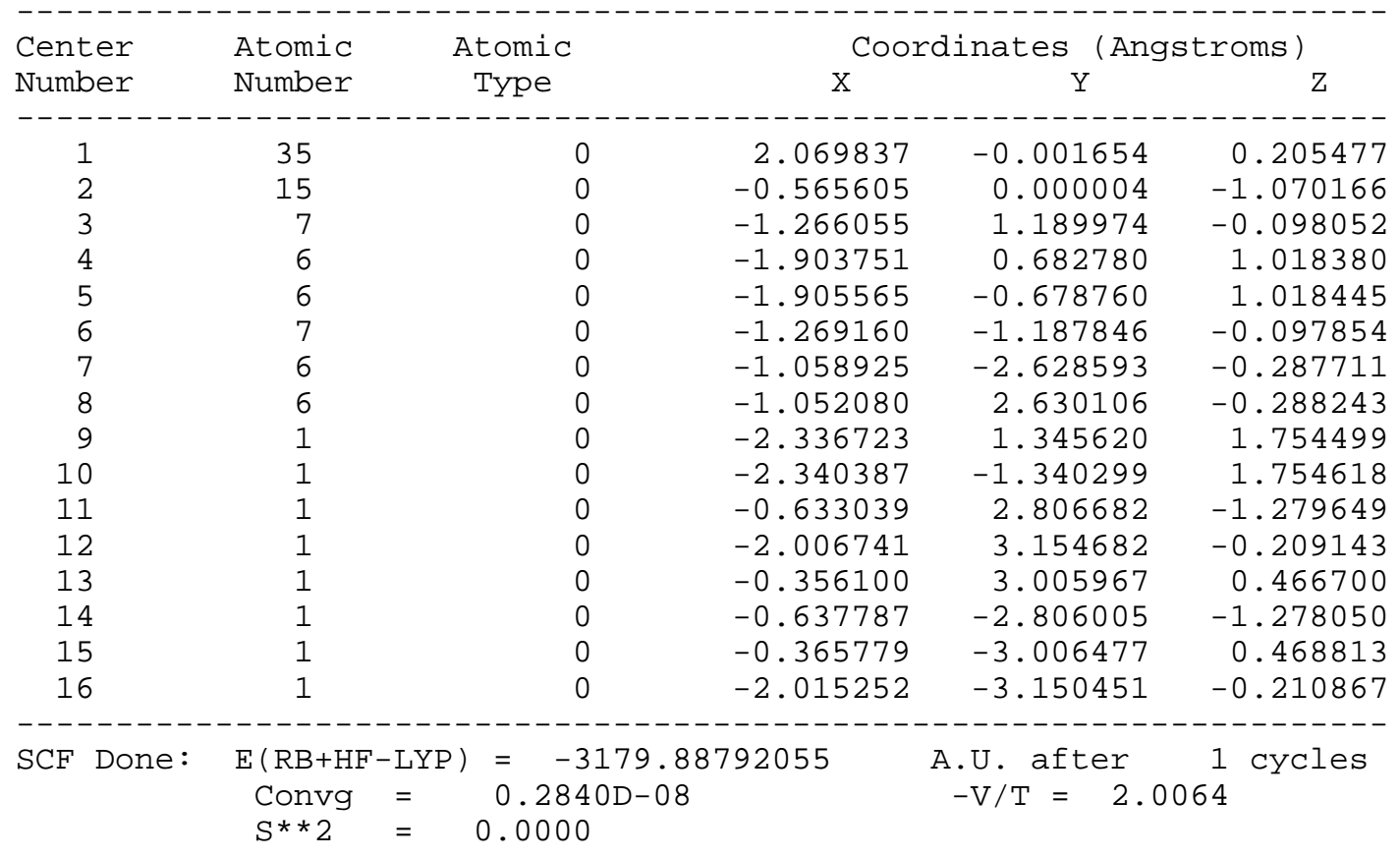

Variational C-PCM results

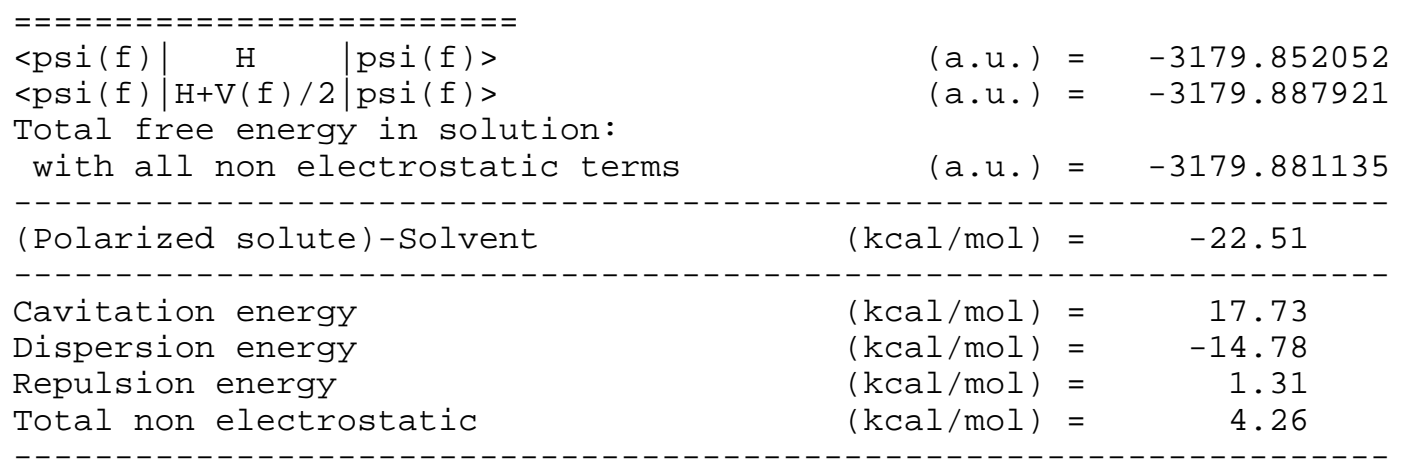

2-Chloro-1,3-Dimethyl-1,3,2-diazaphospholidine 2b[Cl]:

\begin{tabular}{|c|c|c|c|c|c|}
\hline \multirow{2}{*}{$\begin{array}{l}\text { Center } \\
\text { Number }\end{array}$} & Atomic & Atomic & \multicolumn{3}{|c|}{ Coordinates (Angstroms) } \\
\hline & Number & Tyре & $X$ & $\mathrm{Y}$ & Z \\
\hline & & & & . & -- \\
\hline 1 & 6 & $\odot$ & -0.231679 & -1.613917 & 0.884200 \\
\hline 2 & 6 & 0 & 1.270727 & -1.335153 & 0.746466 \\
\hline 3 & 7 & 0 & 1. 381992 & -0.181461 & -0.164834 \\
\hline 4 & 15 & $\odot$ & -0.023245 & 0.280817 & -0.918585 \\
\hline 5 & 7 & $\odot$ & -0.856787 & -1.053778 & -0.330643 \\
\hline 6 & 17 & 0 & -0.889319 & 2.052102 & $\odot .566349$ \\
\hline 7 & 6 & ○ & 2.671594 & 0.480357 & -0.313806 \\
\hline 8 & 6 & $\odot$ & -2.312011 & -1.155563 & $-\odot .429523$ \\
\hline 9 & 1 & $\odot$ & $-\odot .445092$ & -2.684593 & $\odot .95 \odot 227$ \\
\hline 10 & 1 & $\odot$ & -0.648793 & -1.119759 & 1.773452 \\
\hline 11 & 1 & $\odot$ & 1.725856 & -1.102689 & 1.715252 \\
\hline 12 & 1 & $\odot$ & 1.805069 & -2.187887 & $\odot .307483$ \\
\hline 13 & 1 & $\odot$ & 3.435539 & -0.246994 & -0.612850 \\
\hline 14 & 1 & $\odot$ & 2.608855 & 1.252324 & -1.084425 \\
\hline
\end{tabular}




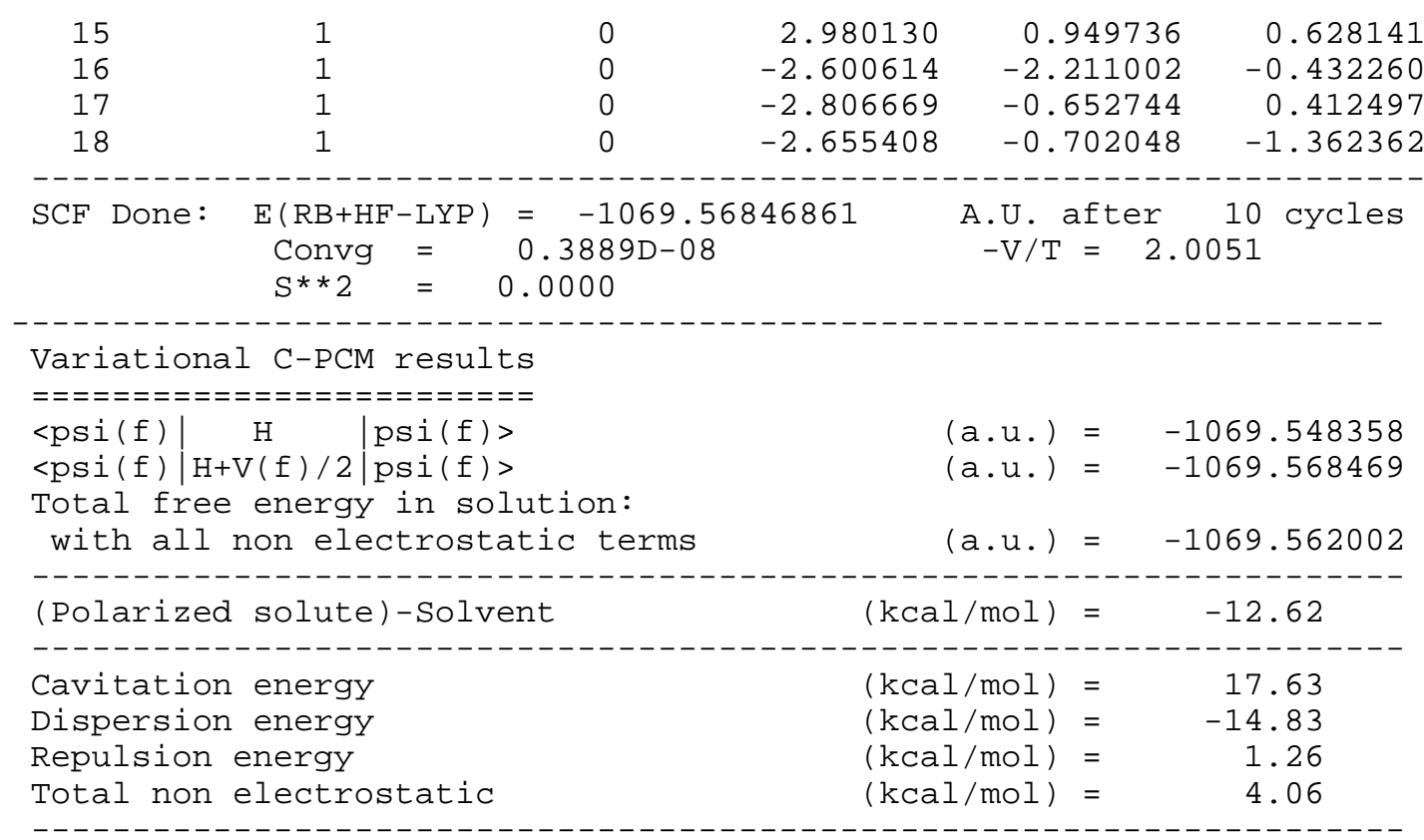

\section{Chloro-bis-(dimethylamino)-phosphine $3 \mathrm{~b}[\mathrm{Cl}]$ :}

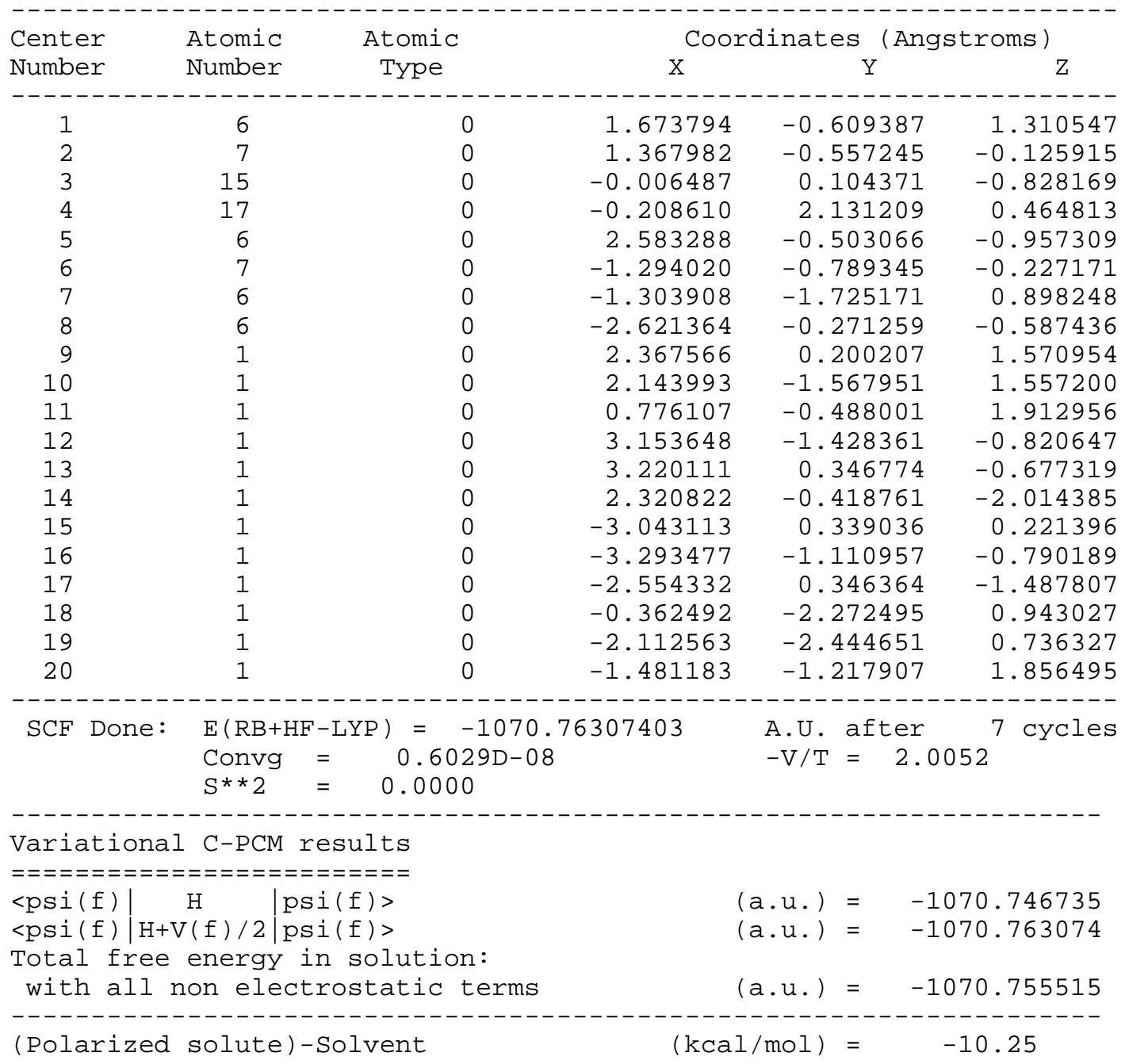




$\begin{array}{lrr}\text { Cavitation energy } & (\mathrm{kcal} / \mathrm{mol})= & 18.79 \\ \text { Dispersion energy } & (\mathrm{kcal} / \mathrm{mol})= & -15.27 \\ \text { Repulsion energy } & (\mathrm{kcal} / \mathrm{mol})= & 1.22 \\ \text { Total non electrostatic } & (\mathrm{kcal} / \mathrm{mol})= & 4.74\end{array}$

\section{F-bridged 2-Fluoro-1,3-Dimethyl-1,3,2-diazaphospholene / 1,3-Dimethyl-1,3,2-}

\section{diazaphospholenium Complex 4'b[F]:}

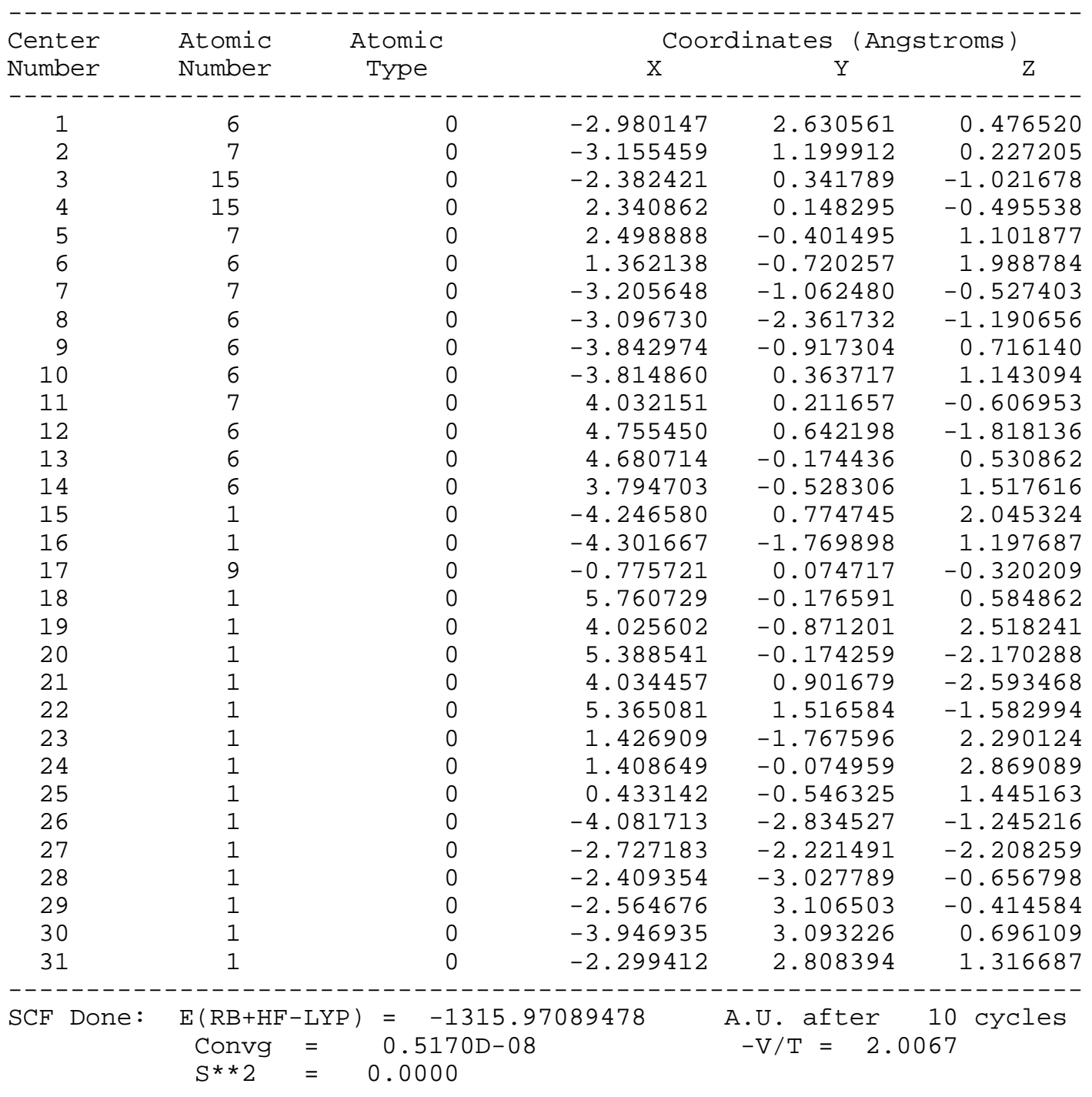

Variational C-PCM results

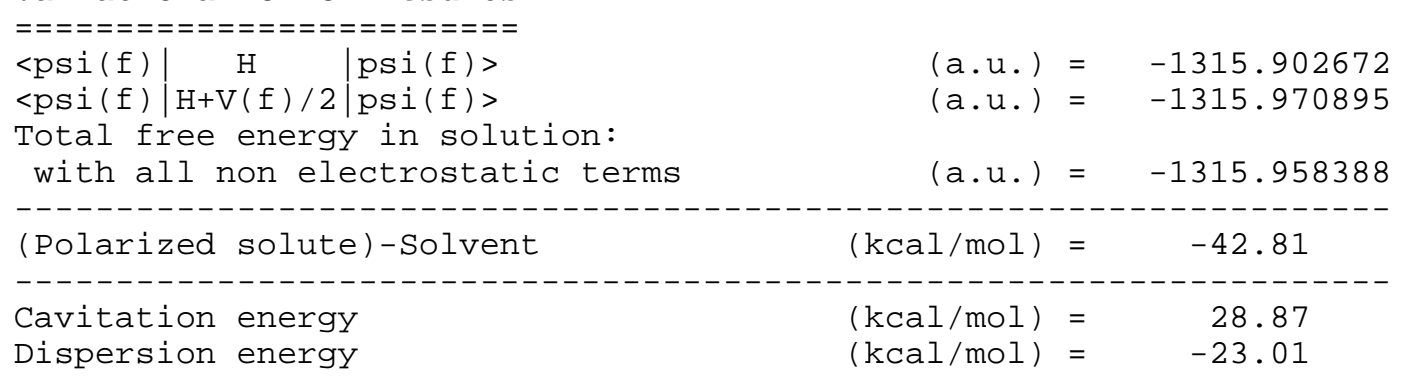


F-bridged 2-Fluoro-1,3-Dimethyl-1,3,2-diazaphospholene / 1,3-Dimethyl-1,3,2-

diazaphospholenium Complex $4 \mathrm{~b}[\mathrm{~F}]\left(\mathrm{C}_{2}\right.$-symmetric Transition State):

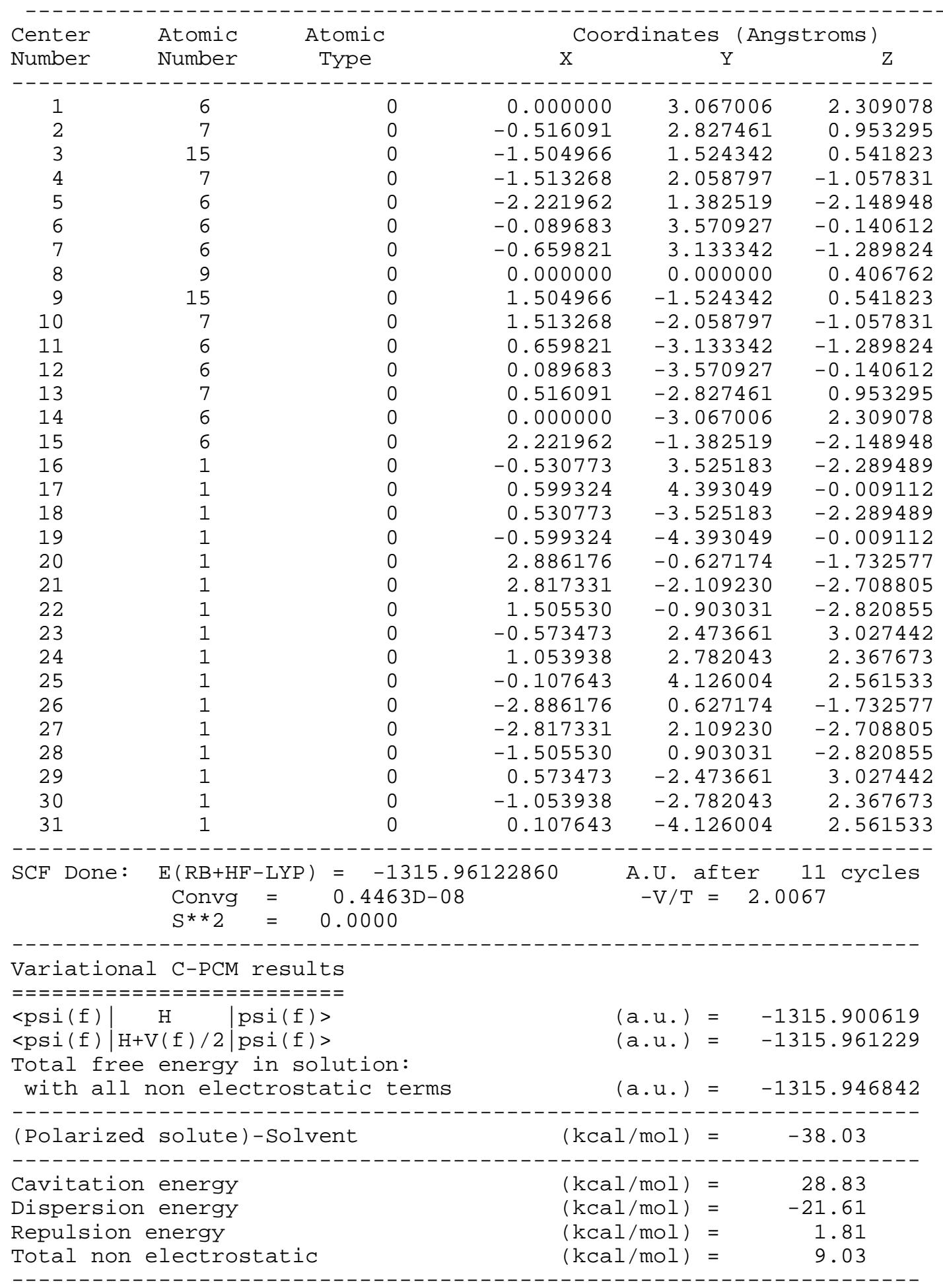




\section{Cl-bridged 2-Chloro-1,3-Dimethyl-1,3,2-diazaphospholene / 1,3-Dimethyl-1,3,2-}

\section{diazaphospholenium Complex 4b[Cl]:}

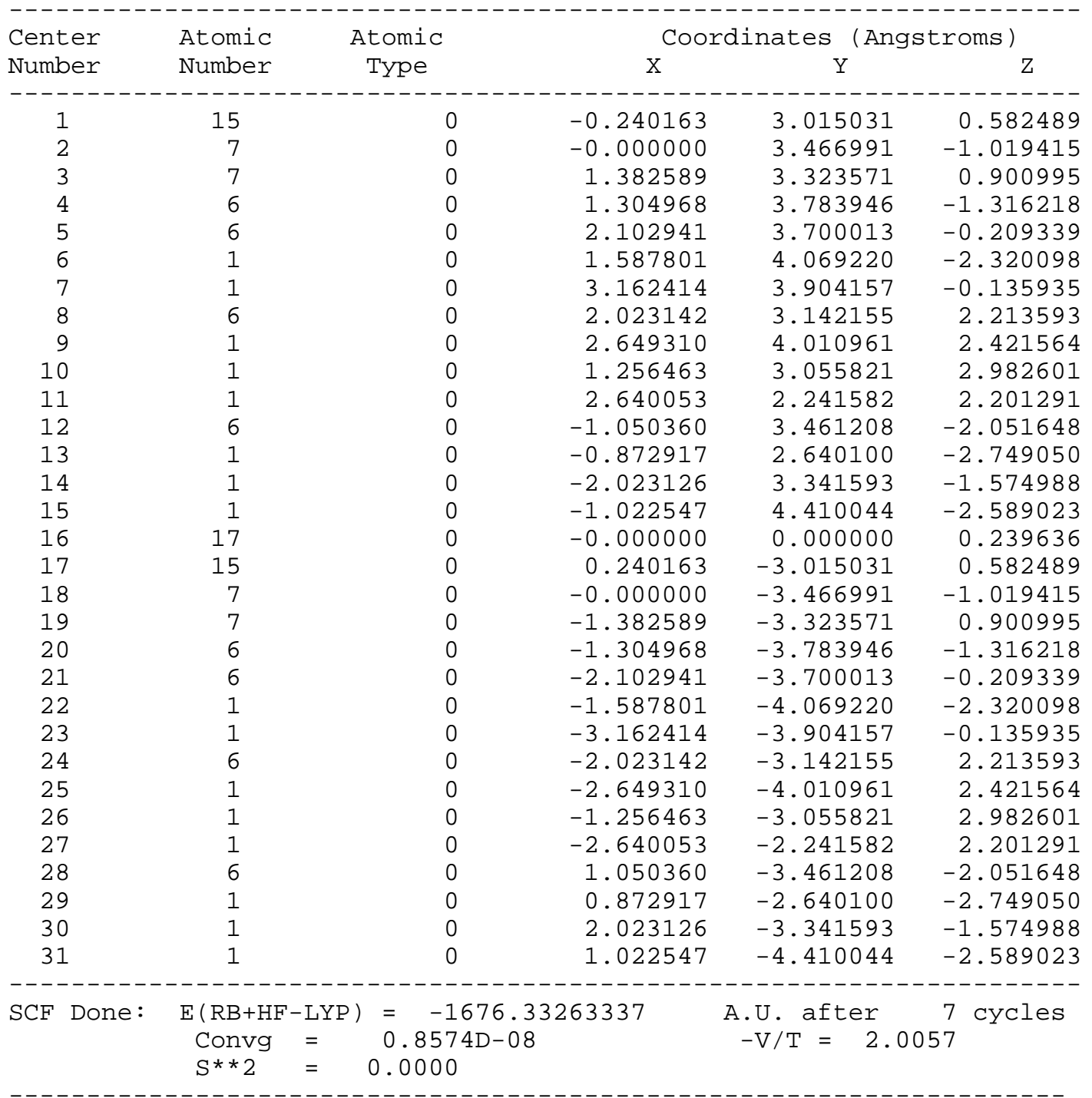

Variational C-PCM results

\begin{tabular}{|c|c|c|}
\hline 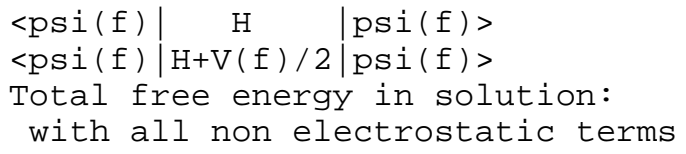 & $\begin{array}{l}(\mathrm{a} \cdot \mathrm{u} .) \\
(\mathrm{a} \cdot \mathrm{u} .)\end{array}$ & $\begin{array}{l}-1676.257603 \\
-1676.332633\end{array}$ \\
\hline (Polarized solute)-Solvent & $(\mathrm{kcal} / \mathrm{mol})$ & -47.08 \\
\hline $\begin{array}{l}\text { Cavitation energy } \\
\text { Dispersion energy } \\
\text { Repulsion energy } \\
\text { Total non electrostatic }\end{array}$ & $\begin{array}{l}(\mathrm{kcal} / \mathrm{mol})= \\
(\mathrm{kcal} / \mathrm{mol})= \\
(\mathrm{kcal} / \mathrm{mol})= \\
(\mathrm{kcal} / \mathrm{mol})=\end{array}$ & $\begin{array}{r}31.06 \\
-23.71 \\
2.05 \\
9.40\end{array}$ \\
\hline
\end{tabular}


Cl-bridged 2-Chloro-1,3-Dimethyl-1,3,2-diazaphospholidine / 1,3-Dimethyl-1,3,2-

diazaphospholidinium Complex 5b[Cl]:

\begin{tabular}{|c|c|c|c|c|c|}
\hline \multirow{2}{*}{$\begin{array}{l}\text { Center } \\
\text { Number }\end{array}$} & \multirow{2}{*}{$\begin{array}{l}\text { Atomic } \\
\text { Number }\end{array}$} & Atomic & \multicolumn{3}{|c|}{ Coordinates (Angstroms) } \\
\hline & & Type & $x$ & Y & Z \\
\hline 1 & 6 & & 2 & 2605100 & - 172209 \\
\hline $\begin{array}{l}1 \\
2\end{array}$ & $\begin{array}{l}6 \\
7\end{array}$ & $\begin{array}{l}\Theta \\
\odot\end{array}$ & $\begin{array}{l}-2.897089 \\
-3.155126\end{array}$ & $\begin{array}{l}-2.605108 \\
-1.165585\end{array}$ & $\begin{array}{l}-0.1 / 2299 \\
-0.150789\end{array}$ \\
\hline 3 & 15 & $\odot$ & -2.172549 & -0.043938 & -0.873383 \\
\hline 4 & 17 & $\odot$ & -0.020798 & -0.250367 & $\odot .810124$ \\
\hline 5 & 15 & $\odot$ & 2.178067 & 0.099353 & -0.869346 \\
\hline 6 & 7 & $\odot$ & 3.022724 & 1.227797 & $-\odot .018640$ \\
\hline 7 & 6 & $\odot$ & 2.806247 & 2.670574 & $-\odot .079096$ \\
\hline 8 & 7 & $\odot$ & -2.970891 & 1.234135 & -0.205477 \\
\hline 9 & 6 & $\odot$ & -2.701687 & 2.642493 & -0.481820 \\
\hline 10 & 6 & $\odot$ & -4.085738 & 0.883553 & $\odot .699335$ \\
\hline 11 & 6 & $\Theta$ & -3.949505 & -0.621649 & 0.971421 \\
\hline 12 & 7 & $\odot$ & 3.110554 & -1.157633 & $-\odot .329269$ \\
\hline 13 & 6 & $\odot$ & 2.781844 & -2.564836 & $-\odot .557343$ \\
\hline 14 & 6 & $\odot$ & 3.945899 & -0.821735 & $\odot .844184$ \\
\hline 15 & 6 & $\odot$ & 4.127903 & 0.703596 & $\odot .811732$ \\
\hline 16 & 1 & $\odot$ & -4.921920 & -1.119607 & 1.008597 \\
\hline 17 & 1 & $\odot$ & -3.419038 & -0.818193 & 1.912685 \\
\hline 18 & 1 & $\odot$ & -5.031645 & 1.125673 & $\odot .199639$ \\
\hline 19 & 1 & $\odot$ & -4.018076 & 1.469110 & 1.620846 \\
\hline 20 & $\overline{1}$ & $\odot$ & 3.429726 & -1.150422 & 1.755741 \\
\hline 21 & $\overline{1}$ & $\odot$ & 4.901674 & -1.348064 & $\odot .777085$ \\
\hline 22 & 1 & $\odot$ & 4.079866 & 1.136989 & 1.815000 \\
\hline 23 & 1 & $\odot$ & 5.079015 & 0.994672 & $\odot .349999$ \\
\hline 24 & 1 & $\Theta$ & 2.459846 & 3.044734 & 0.890320 \\
\hline 25 & 1 & $\odot$ & 3.742352 & 3.174324 & -0.342654 \\
\hline 26 & 1 & 0 & 2.059102 & 2.906975 & -0.839829 \\
\hline 27 & 1 & 0 & 3.705441 & -3.133210 & $-\odot .699133$ \\
\hline 28 & 1 & $\odot$ & 2.233904 & -2.977617 & ๑. 298261 \\
\hline 29 & 1 & $\odot$ & 2.170679 & -2.663831 & -1.456954 \\
\hline 30 & 1 & $\odot$ & -3.608404 & 3.126713 & -0.860393 \\
\hline 31 & 1 & $\odot$ & -2.379309 & 3.153327 & $\odot .431757$ \\
\hline 32 & 1 & $\odot$ & -1.917406 & 2.734418 & -1.236285 \\
\hline 33 & 1 & $\odot$ & -3.850075 & -3.141304 & $-\odot .198436$ \\
\hline 34 & 1 & $\odot$ & -2.325474 & -2.868685 & -1.064629 \\
\hline 35 & $\overline{1}$ & 0 & -2.337526 & -2.913314 & $\odot .719765$ \\
\hline SCF Done: & $\begin{array}{c}E(R B+H F-L Y P) \\
\text { Convg }= \\
S^{*} 2=\end{array}$ & $\begin{array}{rr}= & -1 \\
0.413 \\
0.0000\end{array}$ & 82434 & $\begin{array}{l}\text { A.U. after } \\
-\mathrm{V} / \mathrm{T}=2 .\end{array}$ & 10 cycles \\
\hline
\end{tabular}

Variational C-PCM results

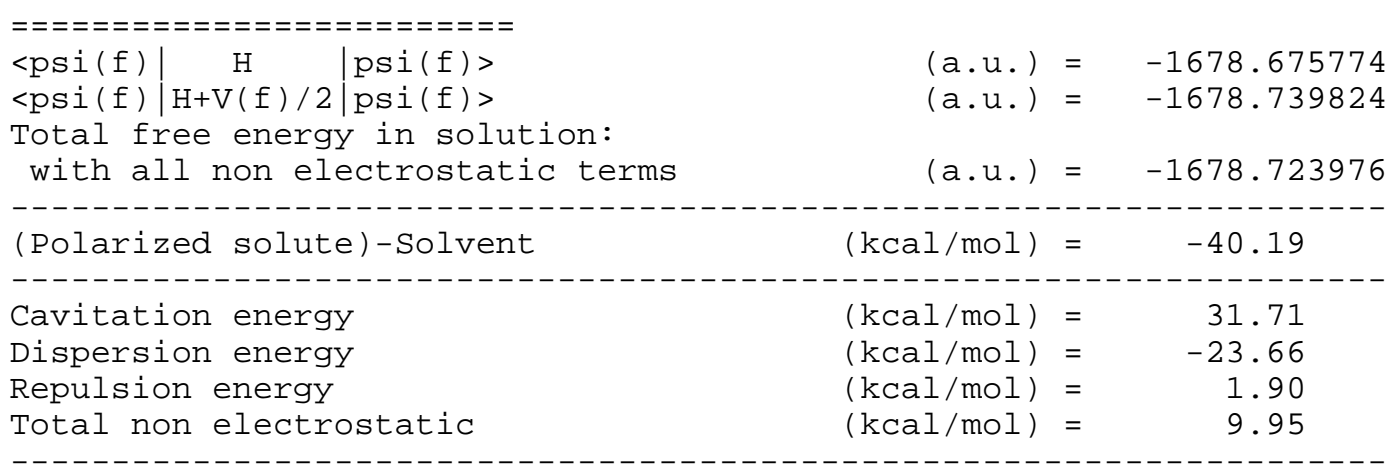


Cl-bridged Chloro-bis(dimethylamino)-phosphine / bis(dimethylamino)-phosphenium Complex 6b[Cl]:

\begin{tabular}{|c|c|c|c|c|c|}
\hline \multirow{2}{*}{$\begin{array}{l}\text { Center } \\
\text { Number }\end{array}$} & \multirow{2}{*}{$\begin{array}{l}\text { Atomic } \\
\text { Number }\end{array}$} & Atomic & \multicolumn{3}{|c|}{ Coordinates (Angstroms) } \\
\hline & & Type & $x^{200}$ & ${ }_{Y}$ & Z \\
\hline & & & & & \\
\hline 1 & 6 & $\odot$ & 3.100669 & -2.059979 & -0.470754 \\
\hline 2 & 6 & $\odot$ & 2.929204 & -1.826398 & 1.970841 \\
\hline 3 & 7 & $\odot$ & 2.965846 & -1.138502 & $\odot .667117$ \\
\hline 4 & 6 & $\Theta$ & 2.603368 & 2.522999 & -0.939690 \\
\hline 5 & 6 & $\Theta$ & 4.161447 & $\odot .702144$ & -1.542158 \\
\hline 6 & 7 & 0 & 3.151758 & 1.197781 & $-\odot .600800$ \\
\hline 7 & 15 & $\odot$ & 2.416563 & ○. 424830 & 0.668510 \\
\hline 8 & 15 & 0 & -2.523046 & -0.745411 & $\odot .481285$ \\
\hline 9 & 7 & $\odot$ & -3.291072 & -0.687827 & -0.980193 \\
\hline 10 & 6 & $\odot$ & -3.434085 & $\odot .441788$ & -1.912887 \\
\hline 11 & 6 & $\odot$ & -3.633024 & -1.997052 & -1.574209 \\
\hline 12 & 7 & $\odot$ & -2.809965 & 0.669839 & 1.281424 \\
\hline 13 & 6 & $\Theta$ & -1.966000 & $\odot .896193$ & 2.468502 \\
\hline 14 & 6 & $\odot$ & -3.654587 & 1.811173 & ๑. 910631 \\
\hline 15 & 17 & $\odot$ & ๑. .026692 & -0.011292 & -0.509795 \\
\hline 16 & 1 & $\odot$ & 2.394712 & -2.882631 & -0.339332 \\
\hline 17 & 1 & $\odot$ & 4.114400 & -2.471035 & -0.517833 \\
\hline 18 & 1 & $\odot$ & 2.863620 & -1.560744 & -1.407463 \\
\hline 19 & 1 & $\odot$ & 3.890460 & -2.319922 & 2.143973 \\
\hline 20 & 1 & $\odot$ & 2.132362 & -2.578778 & 1.985968 \\
\hline 21 & 1 & 0 & 2.764978 & -1.108848 & 2.778334 \\
\hline 22 & 1 & $\odot$ & 2.031150 & 2.471164 & -1.872447 \\
\hline 23 & 1 & $\odot$ & 3.426111 & 3.234368 & -1.054860 \\
\hline 24 & 1 & $\odot$ & 1.940024 & 2.875265 & -0.145426 \\
\hline 25 & 1 & $\odot$ & 4.729989 & -0.113490 & -1.097780 \\
\hline 26 & 1 & $\odot$ & 4.848891 & 1.524014 & -1.762265 \\
\hline 27 & 1 & $\odot$ & 3.706846 & 0.368027 & -2.482293 \\
\hline 28 & 1 & $\Theta$ & -4.486447 & 0.717843 & -2.033309 \\
\hline 29 & 1 & $\odot$ & -3.040691 & ๑. 131112 & -2.885562 \\
\hline 30 & 1 & $\odot$ & -2.860834 & 1.300956 & -1.572311 \\
\hline 31 & 1 & $\odot$ & -3.426348 & -2.802361 & -0.869657 \\
\hline 32 & 1 & $\odot$ & -3.053782 & -2.157753 & -2.489042 \\
\hline 33 & 1 & $\odot$ & -4.698534 & -2.025393 & -1.813556 \\
\hline 34 & 1 & $\odot$ & -1.458122 & $-\odot .028135$ & 2.755412 \\
\hline 35 & 1 & $\odot$ & -2.597229 & 1.223576 & 3.299003 \\
\hline 36 & 1 & $\odot$ & -1.209609 & 1.660586 & 2.258860 \\
\hline 37 & 1 & $\odot$ & -4.464550 & 1.490087 & $\odot .257957$ \\
\hline 38 & 1 & $\odot$ & -3.071429 & 2.599805 & $\odot .421747$ \\
\hline 39 & 1 & $\odot$ & -4.090415 & 2.218708 & 1.826981 \\
\hline & & & ----- & -------- & \\
\hline SCF Done: & $E(R B$ & D) = & 805083 & A.U. after & 9 cycles \\
\hline & & 0.9540 & & $-\mathrm{V} / \mathrm{T}=2 . \mathrm{C}$ & \\
\hline & $S * * 2$ & 0.0000 & & & \\
\hline
\end{tabular}

Variational C-PCM results

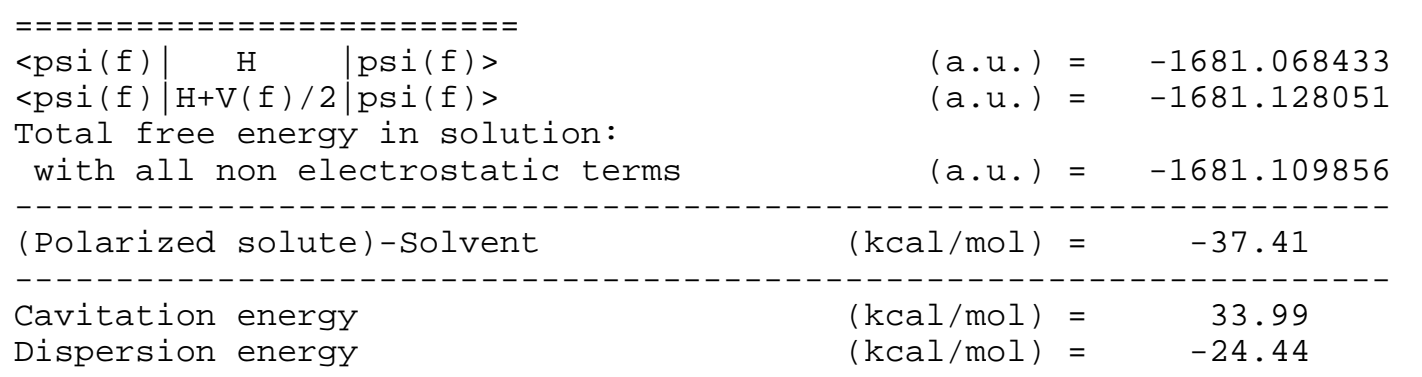


Repulsion energy

$(\mathrm{kcal} / \mathrm{mol})=$

1.87

Total non electrostatic

$(\mathrm{kcal} / \mathrm{mol})=$

11.42

P-P bonded Chloro-bis(dimethylamino)-phosphine / bis(dimethylamino)-phosphenium Complex

\section{$6^{\prime} \mathrm{b}[\mathrm{Cl}]:$}

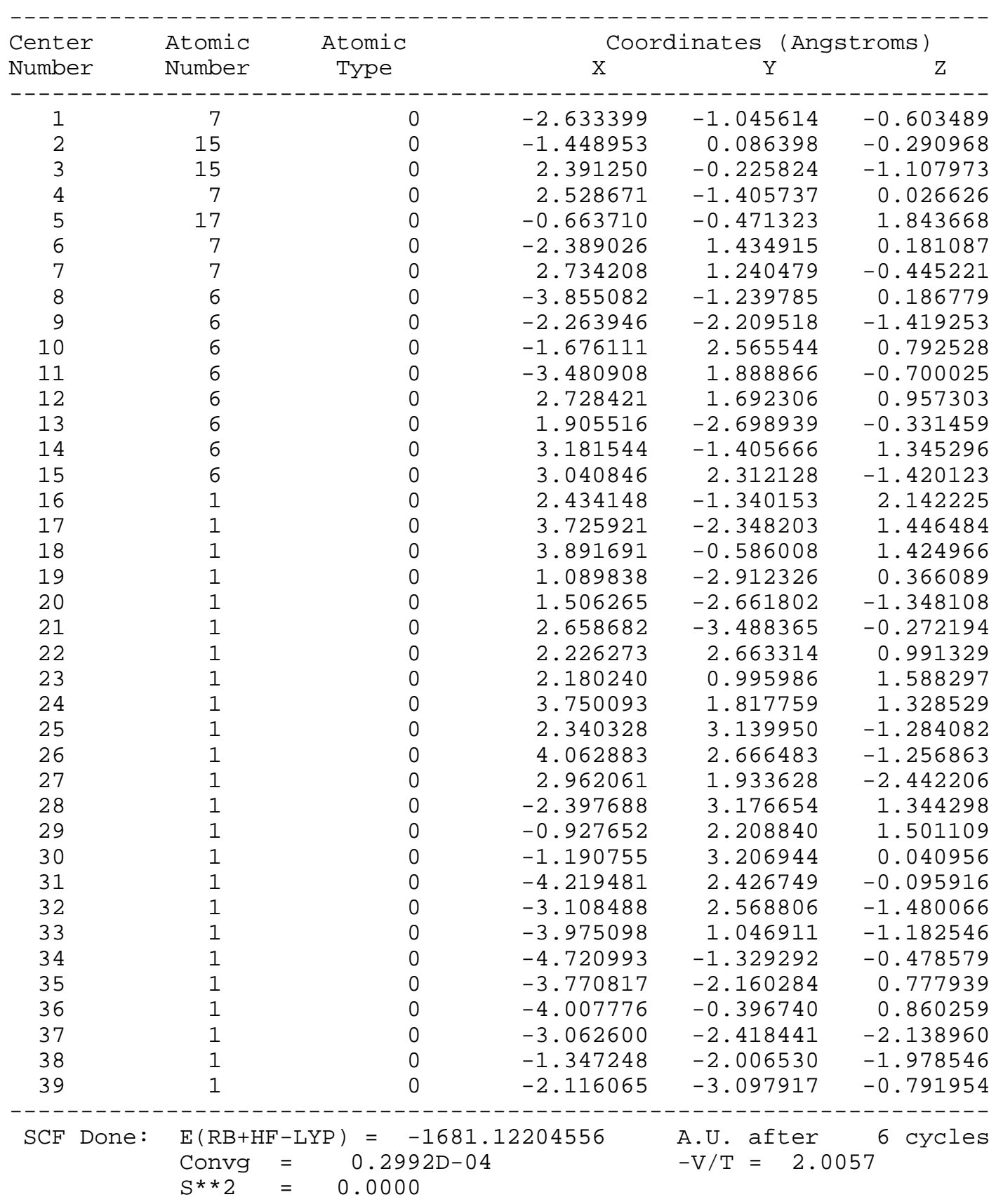

Variational C-PCM results

\begin{tabular}{|c|c|c|}
\hline \\
\hline$|\operatorname{psi}(f)\rangle$ & $(\mathrm{a} \cdot \mathrm{u} \cdot)=$ & -1681.053234 \\
\hline $\begin{array}{l}<p s i(f)|H+V(f) / 2| p s i(f)> \\
\text { Total free energy in solution: }\end{array}$ & $(\mathrm{a} \cdot \mathrm{u})=$. & -1681.122046 \\
\hline with all non electrostatic terms & $($ a.u. $)=$ & -1681.100436 \\
\hline
\end{tabular}




$\begin{array}{lrr}\text { (Polarized solute)-Solvent } & (\mathrm{kcal} / \mathrm{mol})= & -43.18 \\ \text { Cavitation energy } & (\mathrm{kcal} / \mathrm{mol})= & 35.42 \\ \text { Dispersion energy } & (\mathrm{kcal} / \mathrm{mol})= & -23.62 \\ \text { Repulsion energy } & (\mathrm{kcal} / \mathrm{mol})= & 1.76 \\ \text { Total non electrostatic } & (\mathrm{kcal} / \mathrm{mol})= & 13.56\end{array}$

Final atomic coordinates and energies for species in $\mathrm{CH}_{2} \mathrm{Cl}_{2}$ solution at mpw1k/6-31+g** level:

1,3-Dimethyl-1,3,2-diazaphospholenium cation 1b:

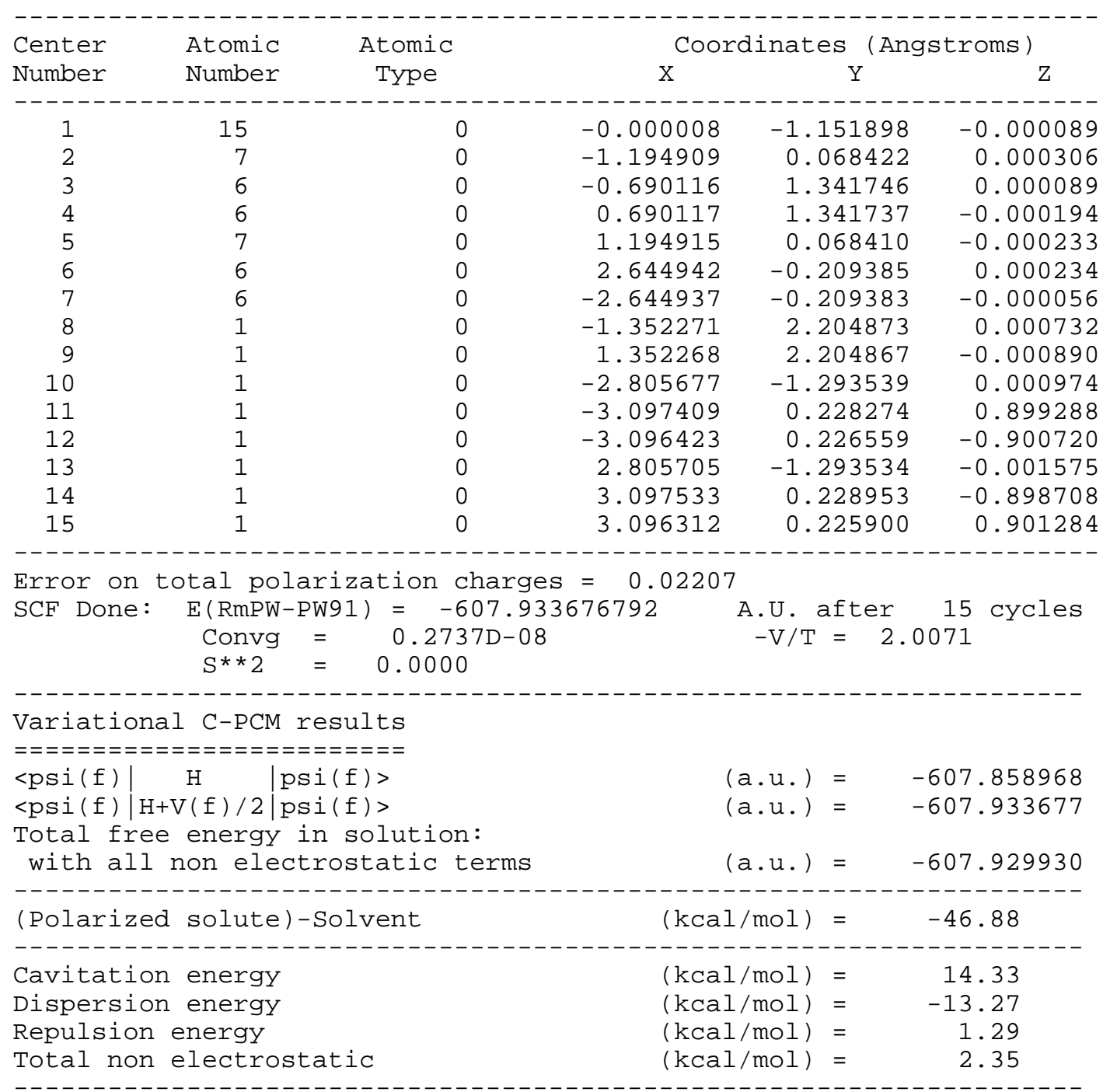

2-Fluoro-1,3-Dimethyl-1,3,2-diazaphospholene 1b[F]:

\begin{tabular}{ccccc} 
Center & Atomic & Atomic & \multicolumn{2}{c}{ Coordinates (Angstroms) } \\
Number & Number & Type & X & Y
\end{tabular}




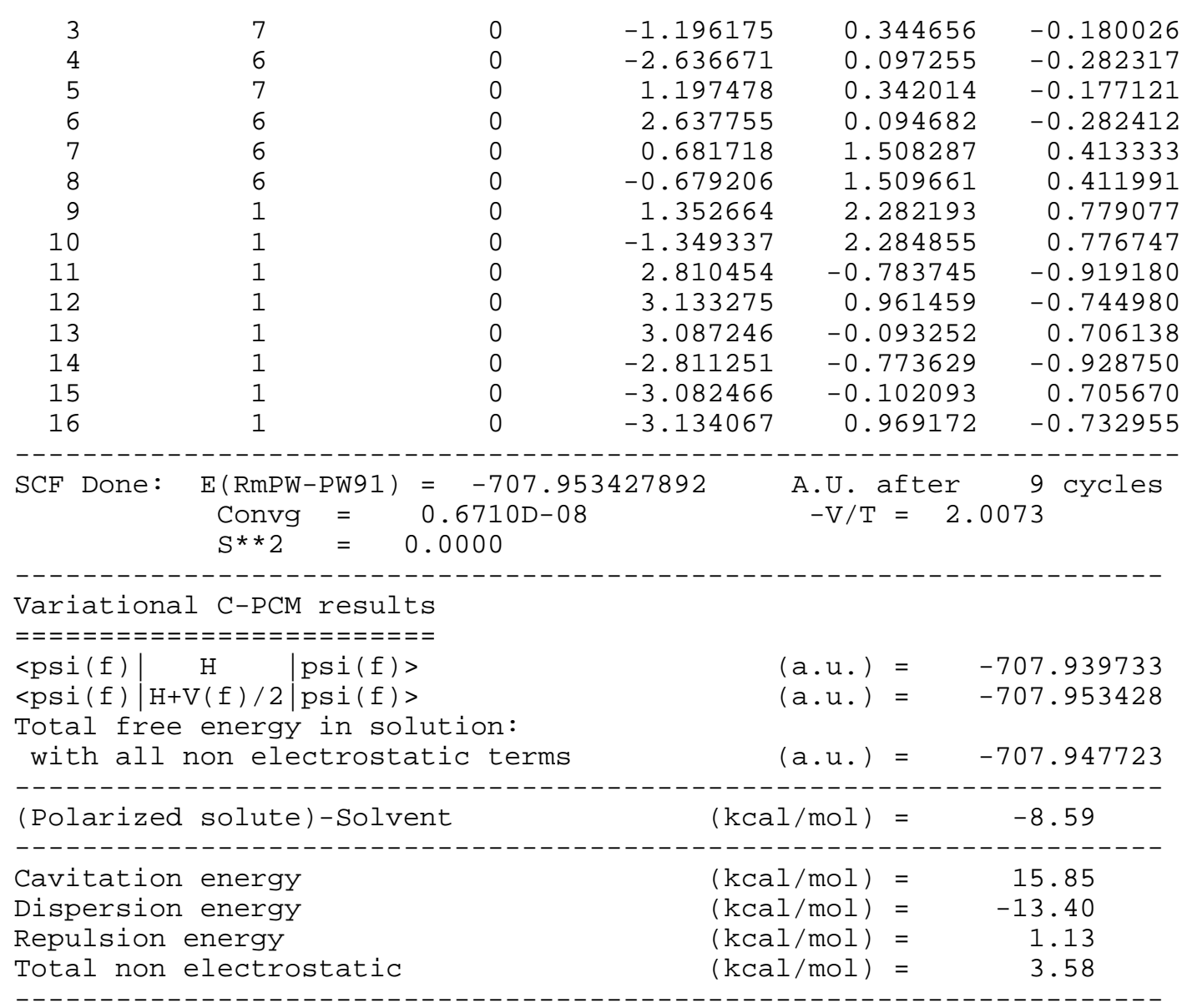

2-Chloro-1,3-Dimethyl-1,3,2-diazaphospholene 1b[Cl]:

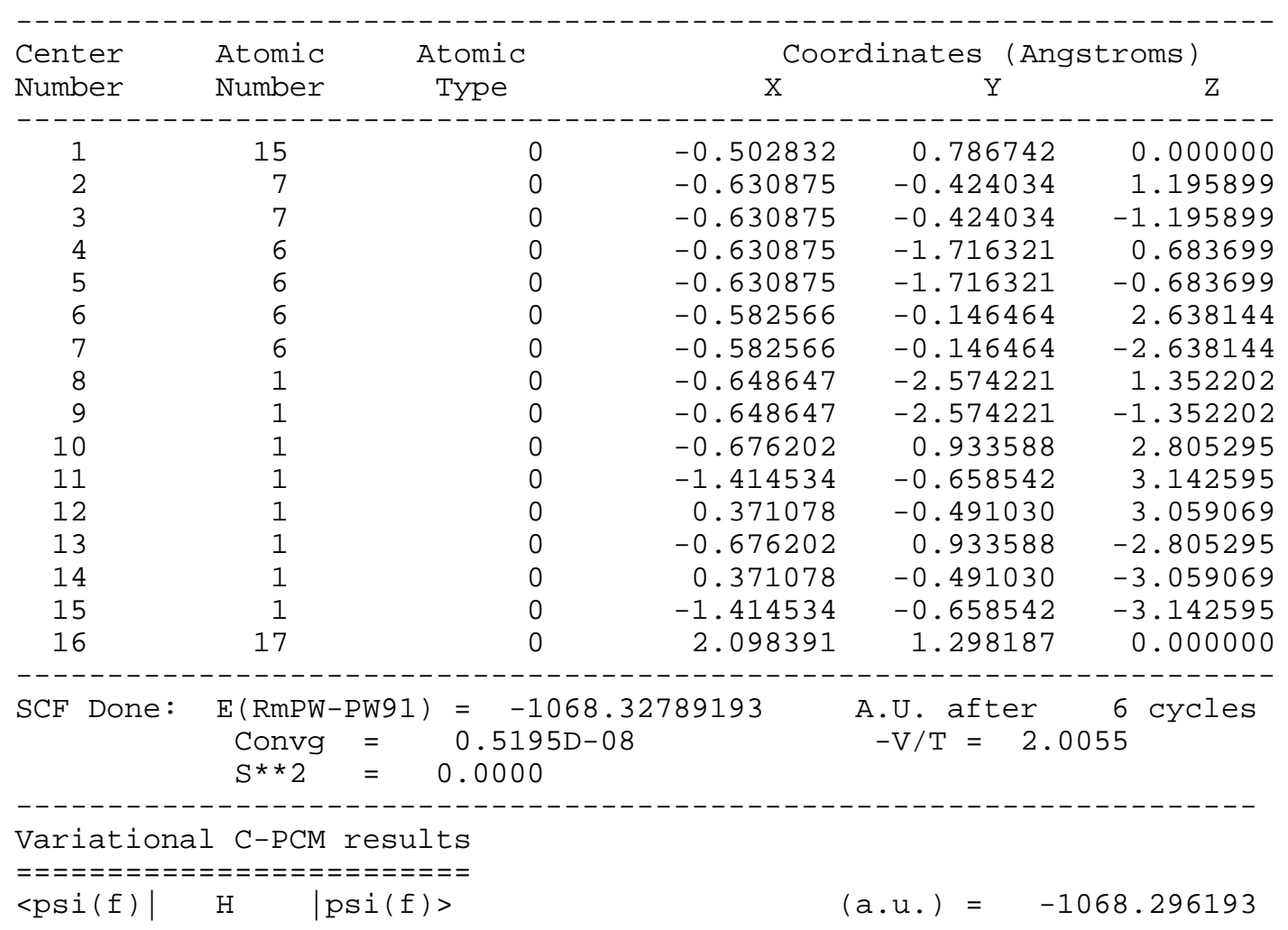




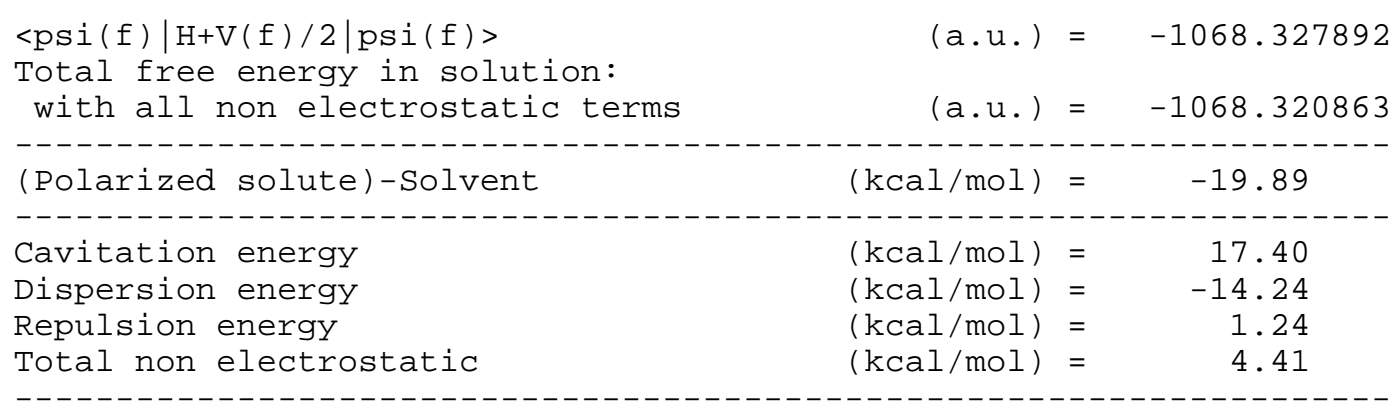

F-bridged 2-Fluoro-1,3-Dimethyl-1,3,2-diazaphospholene / 1,3-Dimethyl-1,3,2-

diazaphospholenium Complex 4'b[F]:

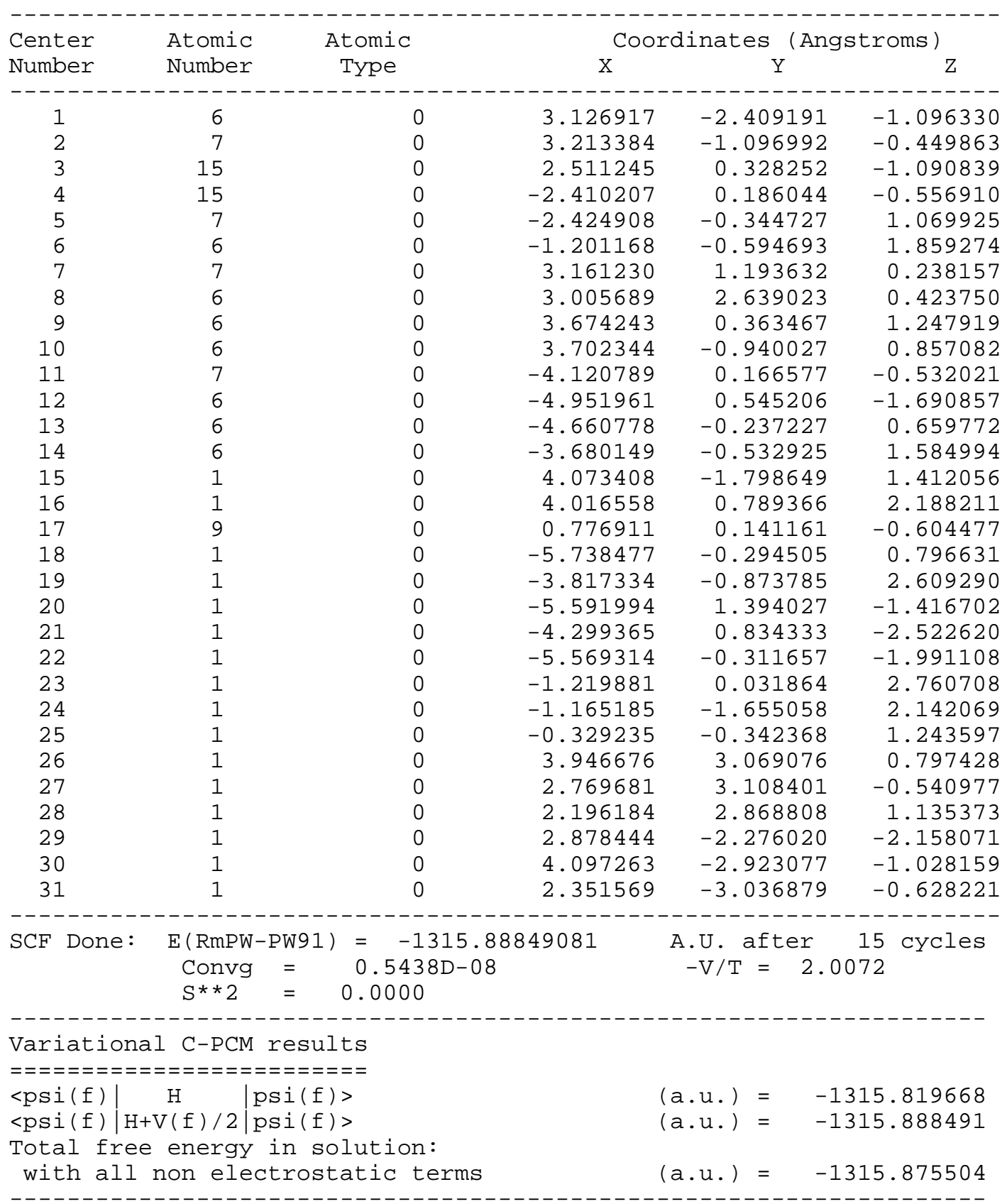




$\begin{array}{lrr}\text { (Polarized solute)-Solvent } & (\mathrm{kcal} / \mathrm{mol})= & -43.19 \\ - & (\mathrm{kcal} / \mathrm{mol})= & 29.12 \\ \text { Cavitation energy } & (\mathrm{kcal} / \mathrm{mol})= & -22.96 \\ \text { Dispersion energy } & (\mathrm{kcal} / \mathrm{mol})= & 1.99 \\ \text { Repulsion energy } & (\mathrm{kcal} / \mathrm{mol})= & 8.15 \\ \text { Total non electrostatic } & & \end{array}$

\section{F-bridged 2-Fluoro-1,3-Dimethyl-1,3,2-diazaphospholene / 1,3-Dimethyl-1,3,2-}

\section{diazaphospholenium Complex $4 \mathrm{~b}[\mathrm{~F}]\left(\mathrm{C}_{2}\right.$-symmetric Transition State):}

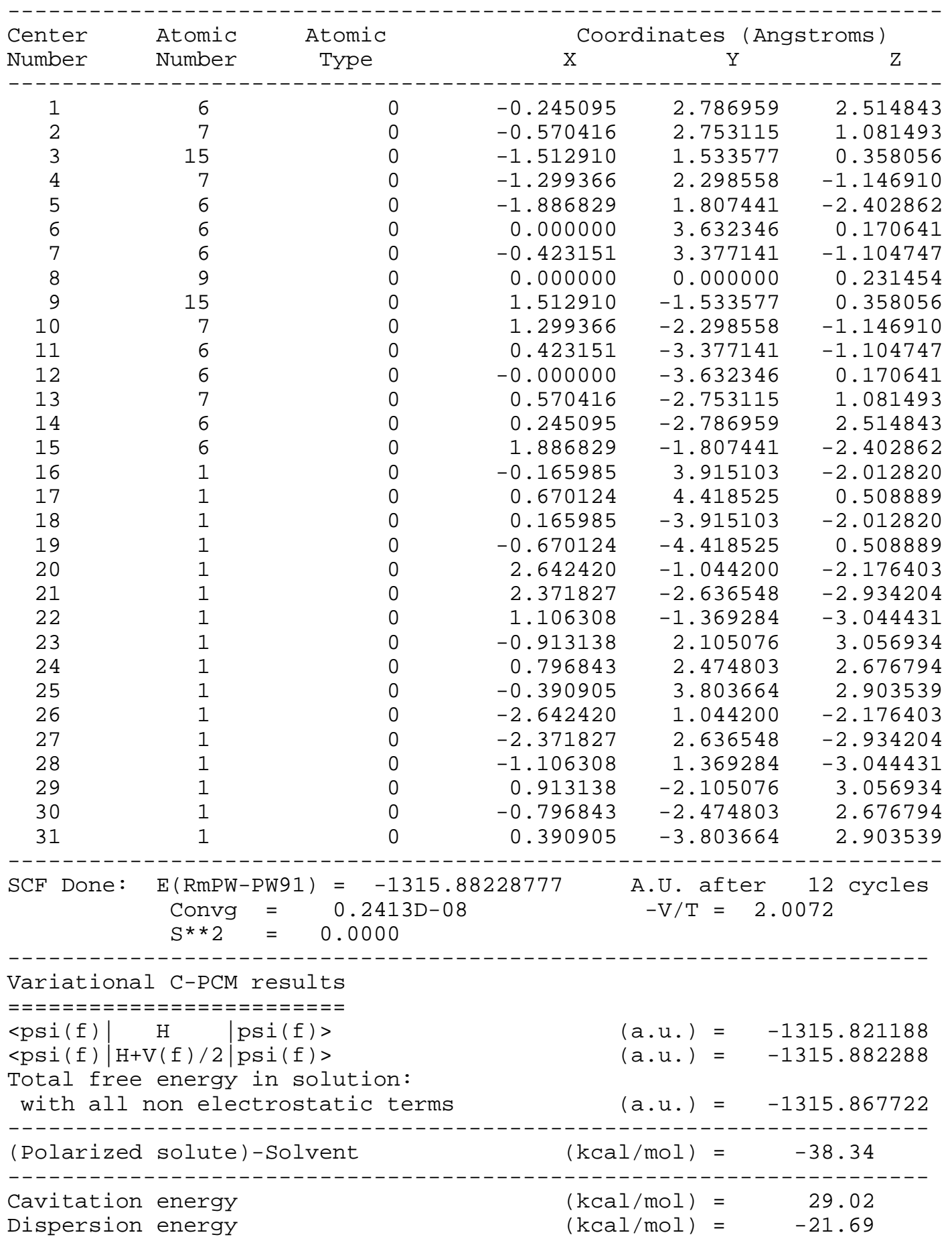


Repulsion energy

$(\mathrm{kcal} / \mathrm{mol})=$

1.82

Total non electrostatic

$(\mathrm{kcal} / \mathrm{mol})=$

9.14

\section{Cl-bridged 2-Chloro-1,3-Dimethyl-1,3,2-diazaphospholene / 1,3-Dimethyl-1,3,2-}

\section{diazaphospholenium Complex 4b[Cl]:}

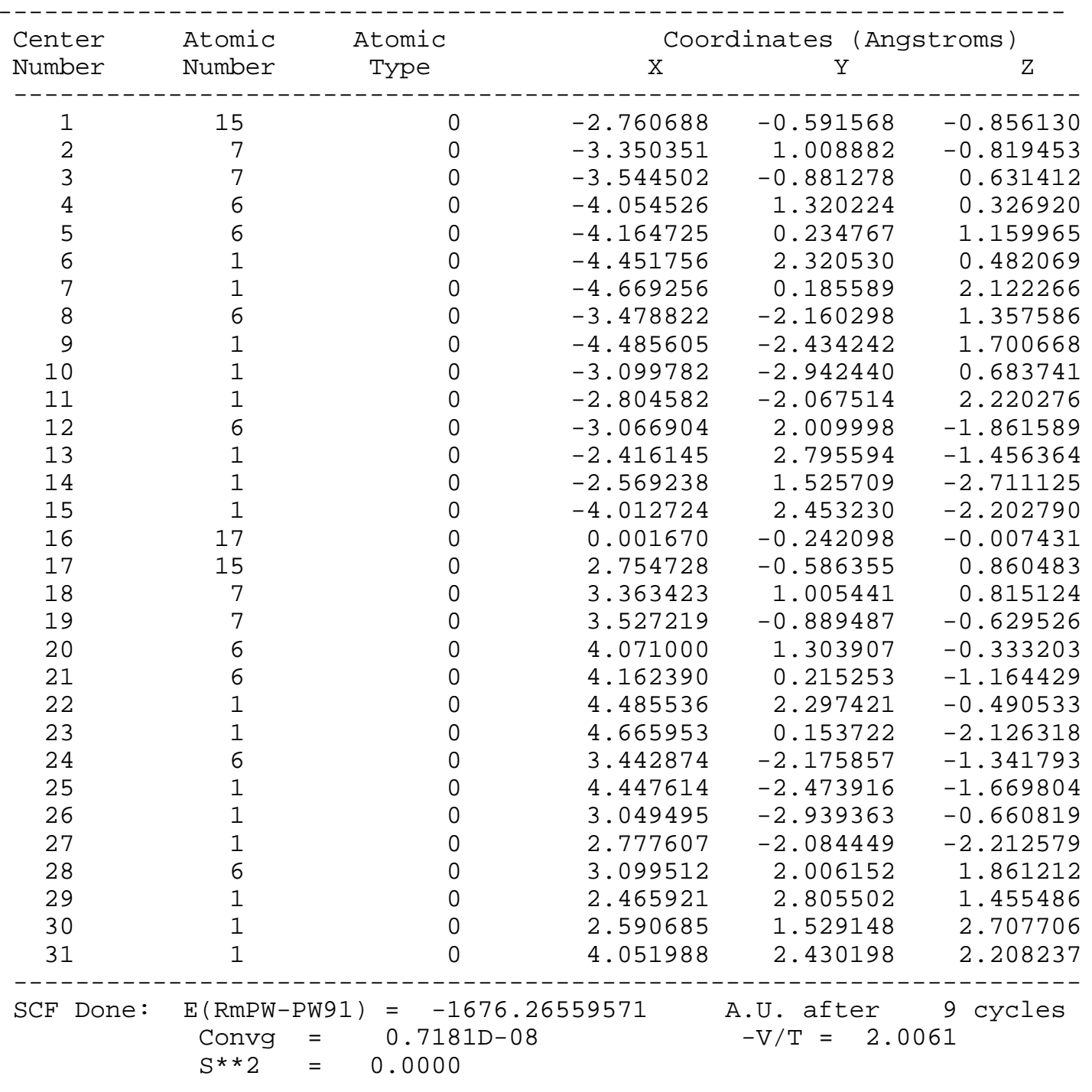

Variational C-PCM results

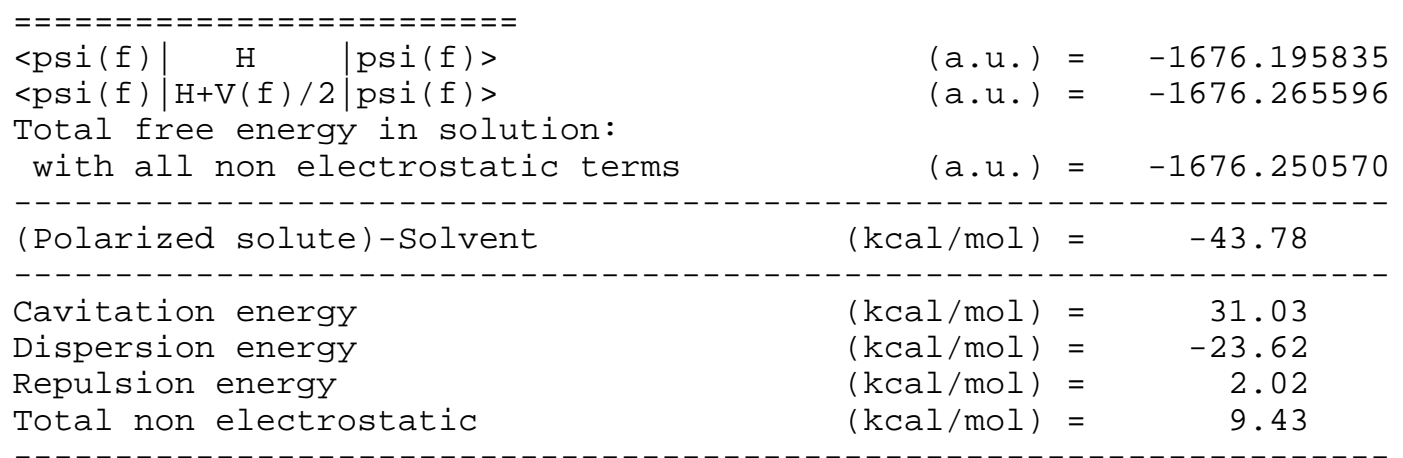


Final atomic coordinates and energies for species in $\mathrm{CH}_{2} \mathrm{Cl}_{2}$ solution at $\mathrm{mp2/6-31}+\mathrm{g}^{* *}$ level:

\section{1,3-Dimethyl-1,3,2-diazaphospholenium cation 1b:}

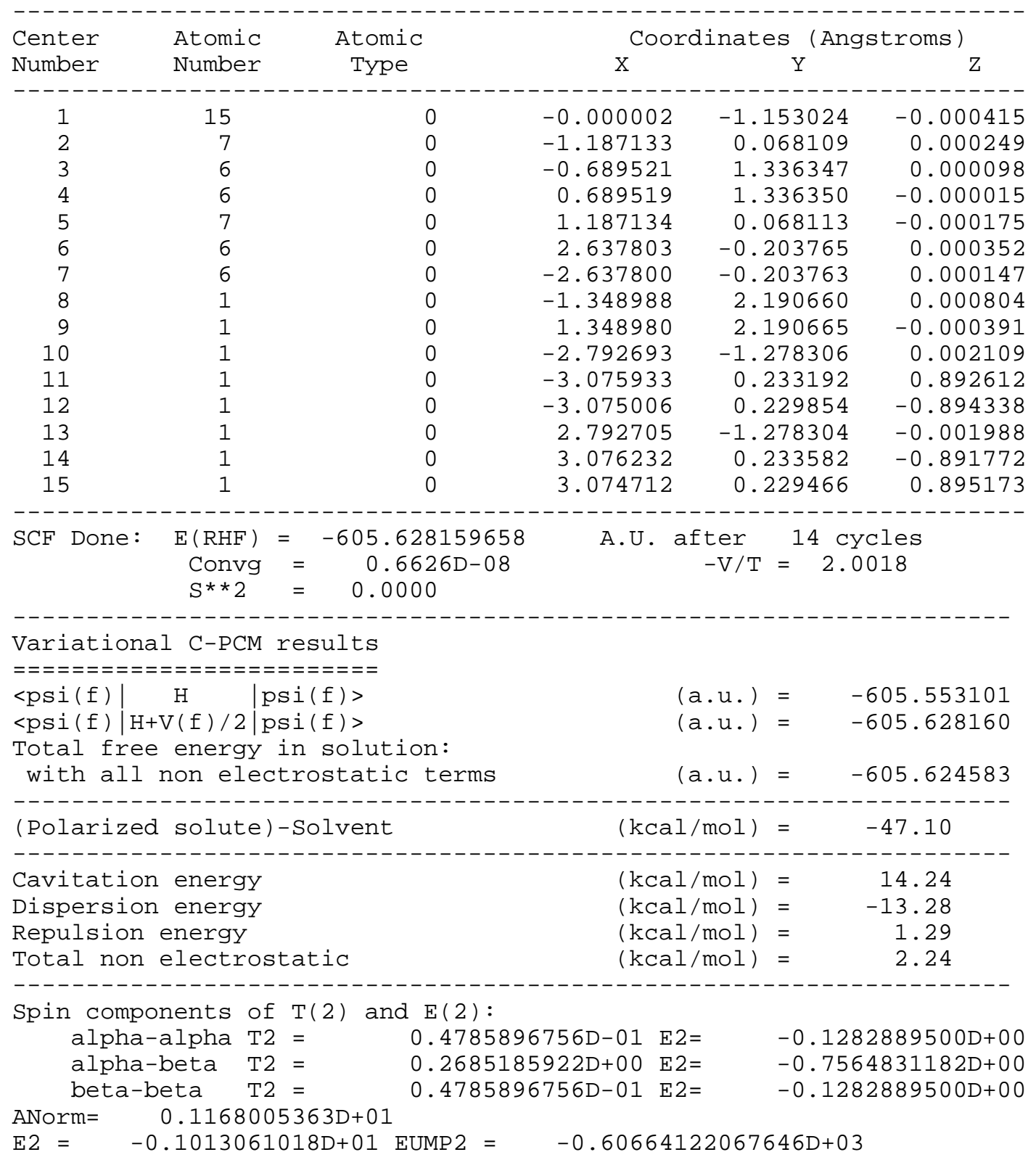

\section{2-Fluoro-1,3-Dimethyl-1,3,2-diazaphospholene 1b[F]:}

\begin{tabular}{|c|c|c|c|c|c|}
\hline \multirow{2}{*}{$\begin{array}{l}\text { Center } \\
\text { Number }\end{array}$} & \multirow{2}{*}{$\begin{array}{l}\text { Atomic } \\
\text { Number }\end{array}$} & \multirow{2}{*}{$\begin{array}{l}\text { Atomic } \\
\text { Type }\end{array}$} & \multicolumn{3}{|c|}{ Coordinates (Angstroms) } \\
\hline & & & $\mathrm{X}$ & $\mathrm{Y}$ & Z \\
\hline & & & & & 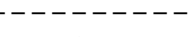 \\
\hline 1 & 9 & $\odot$ & -0.003847 & -1.734385 & 1.011555 \\
\hline 2 & 15 & $\odot$ & 0.000131 & -0.839007 & -0.502052 \\
\hline 3 & 7 & $\odot$ & -1.184662 & 0.337429 & -0.205567 \\
\hline 4 & 6 & $\odot$ & -2.623671 & 0.082649 & -0.276561 \\
\hline 5 & 7 & $\odot$ & 1.185944 & $\odot .335470$ & -0.203895 \\
\hline 6 & 6 & $\odot$ & 2.624791 & 0.080404 & -0.276128 \\
\hline 7 & 6 & $\odot$ & $\odot .679573$ & 1.490510 & 0.409524 \\
\hline
\end{tabular}




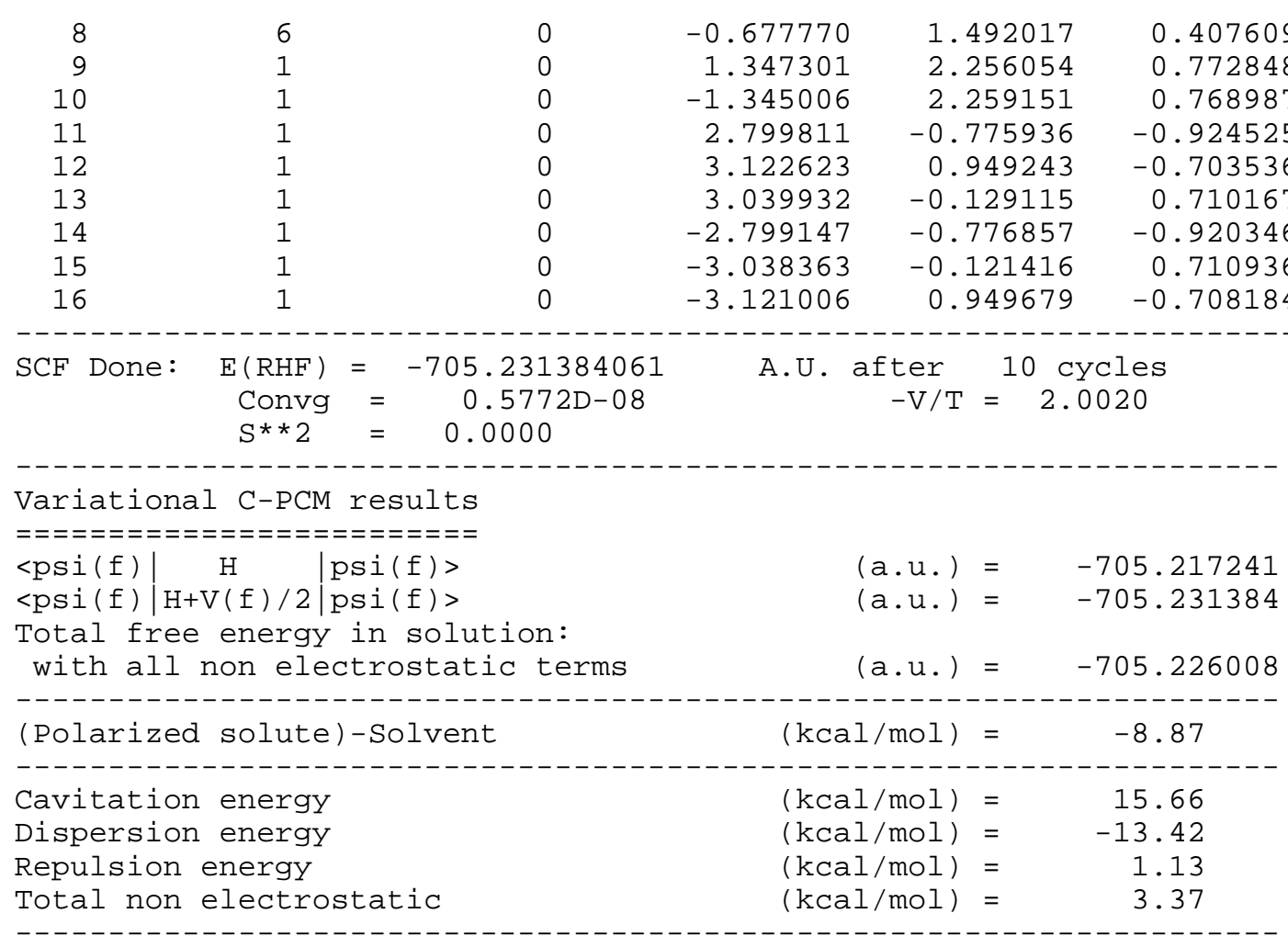

Spin components of $\mathrm{T}(2)$ and $\mathrm{E}(2)$ :
alpha-alpha T2 =
$0.5136028116 \mathrm{D}-01 \mathrm{E} 2=$
alpha-beta $\mathrm{T} 2=$
$0.2884098210 \mathrm{D}+00 \mathrm{E} 2=$
$-0.1536062820 D+00$
beta-beta $\mathrm{T} 2=$
$0.5136028116 \mathrm{D}-01 \mathrm{E} 2=$
$-0.8894368506 \mathrm{D}+00$
$-\odot .1536062820 D+00$

ANorm $=\quad 0.1179461904 \mathrm{D}+01$

$\mathrm{E} 2=-\Theta .1196649415 \mathrm{D}+01$ EUMP2 $=-\odot .70642803347566 \mathrm{D}+03$

\section{2-Chloro-1,3-Dimethyl-1,3,2-diazaphospholene 1b[Cl]:}

\begin{tabular}{|c|c|c|c|c|c|}
\hline \multirow{2}{*}{$\begin{array}{l}\text { Center } \\
\text { Number }\end{array}$} & \multirow{2}{*}{$\begin{array}{l}\text { Atomic } \\
\text { Number }\end{array}$} & \multirow{2}{*}{$\begin{array}{l}\text { Atomic } \\
\text { Type }\end{array}$} & \multicolumn{3}{|c|}{ Coordinates (Angstroms) } \\
\hline & & & $X$ & Y & Z \\
\hline 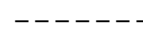 & - - - & & --- - - & -- & ------- \\
\hline 1 & 15 & $\odot$ & -0.007787 & -0.394266 & -1.175611 \\
\hline 2 & 7 & $\odot$ & -1.195413 & -0.743857 & -0.019260 \\
\hline 3 & 7 & $\odot$ & 1.172090 & -0.777775 & -0.020853 \\
\hline 4 & 6 & $\odot$ & -0.704056 & -1.069505 & 1.211732 \\
\hline 5 & 6 & $\odot$ & $\odot .672876$ & -1.088975 & 1.210708 \\
\hline 6 & 6 & $\odot$ & -2.637728 & -0.574415 & -0.255844 \\
\hline 7 & 6 & $\odot$ & 2.618395 & -0.646472 & -0.257221 \\
\hline 8 & 1 & $\odot$ & -1.367860 & -1.301294 & 2.030158 \\
\hline 9 & 1 & $\odot$ & 1.330437 & -1.339406 & 2.028749 \\
\hline 10 & 1 & $\odot$ & -2.796994 & -0.356236 & -1.307809 \\
\hline 11 & 1 & $\odot$ & -3.148594 & -1.496180 & $\odot .008169$ \\
\hline 12 & 1 & $\odot$ & -2.997467 & ๑. 249556 & $\odot .354719$ \\
\hline 13 & 1 & $\odot$ & 2.784123 & -0.423917 & -1.306873 \\
\hline 14 & 1 & 0 & 3.000013 & 0.163808 & ๑.358374 \\
\hline 15 & 1 & $\odot$ & 3.105313 & -1.583126 & -0.000845 \\
\hline 16 & $1 \overline{7}$ & $\odot$ & 0.039657 & 2.525200 & 0.252592 \\
\hline
\end{tabular}

$$
\begin{aligned}
& \text { SCF Done: } E(R H F)=-1065.28016577 \text { A.U. after } 13 \text { cycles } \\
& \text { Convg }=0.3209 \mathrm{D}-08 \quad-\mathrm{V} / \mathrm{T}=2.0013 \\
& \mathrm{~S}^{* * 2}=0.0000
\end{aligned}
$$




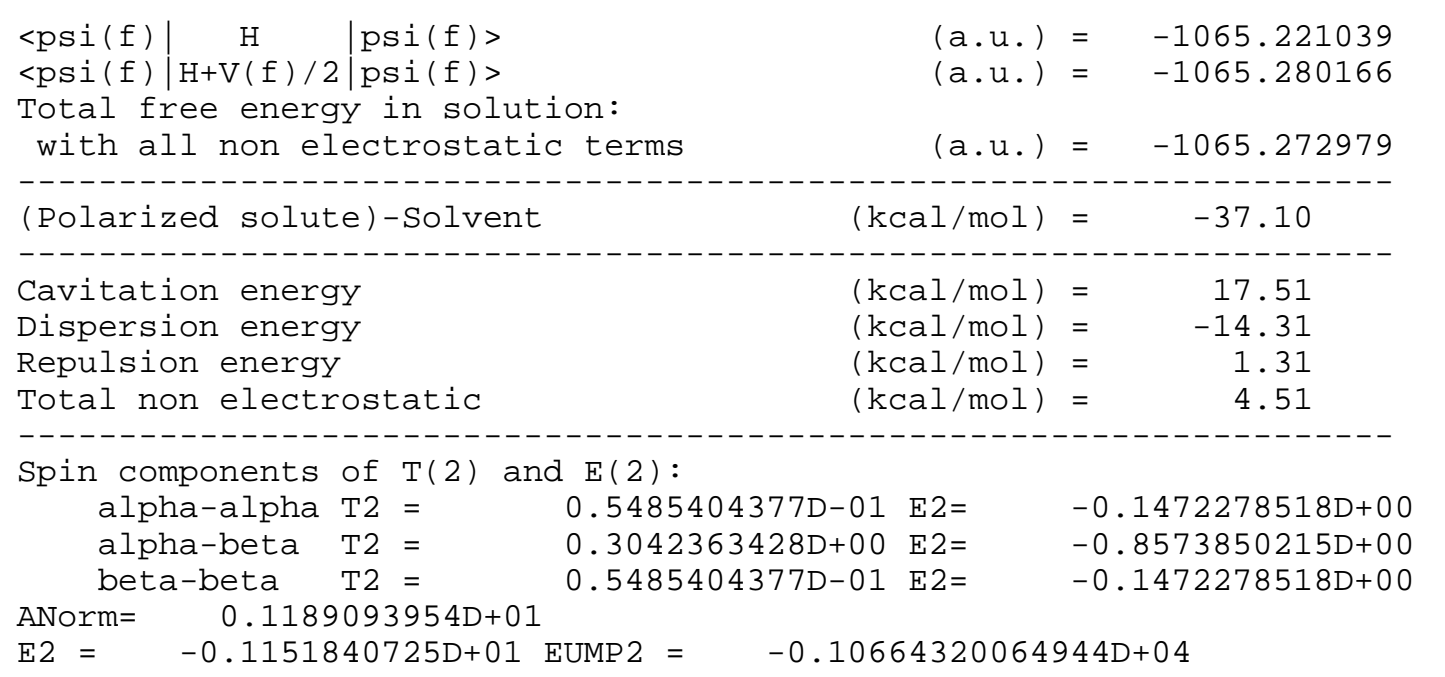

F-bridged 2-Fluoro-1,3-Dimethyl-1,3,2-diazaphospholene / 1,3-Dimethyl-1,3,2-

\section{diazaphospholenium Complex 4'b[F]:}

\begin{tabular}{|c|c|c|c|c|c|}
\hline \multirow{2}{*}{$\begin{array}{l}\text { Center } \\
\text { Number }\end{array}$} & \multirow{2}{*}{$\begin{array}{l}\text { Atomic } \\
\text { Number }\end{array}$} & Atomic & \multicolumn{3}{|c|}{ Coordinates (Angstroms) } \\
\hline & & Type & $\mathrm{X}$ & $\mathrm{Y}$ & Z \\
\hline \multicolumn{6}{|c|}{$-b_{-1}$} \\
\hline 1 & 6 & 0 & -1.269021 & 2.888727 & 0.209246 \\
\hline$\overline{2}$ & 7 & $\odot$ & -1.686067 & 1.504310 & $\odot .426892$ \\
\hline 3 & 15 & 0 & -2.583857 & 0.593289 & -0.682994 \\
\hline 4 & 15 & 0 & 1.522488 & 0.304254 & -0.547230 \\
\hline 5 & 7 & $\odot$ & 1.620949 & -1.359856 & -0.173715 \\
\hline 6 & 6 & $\odot$ & 0.518149 & -2.322026 & -0.369279 \\
\hline 7 & 7 & $\odot$ & -2.635428 & -0.666557 & 0.452866 \\
\hline 8 & 6 & $\Theta$ & -3.323339 & -1.939191 & $\odot .229981$ \\
\hline 9 & 6 & $\Theta$ & -1.743862 & -0.492420 & 1.517880 \\
\hline 10 & 6 & $\odot$ & -1.197415 & 0.752682 & 1.502679 \\
\hline 11 & 7 & $\odot$ & 3.129712 & 0.461454 & $\odot .011228$ \\
\hline 12 & 6 & $\odot$ & 3.857035 & 1.745620 & $\odot .032805$ \\
\hline 13 & 6 & $\odot$ & 3.698637 & -0.696128 & $\odot .447469$ \\
\hline 14 & 6 & $\odot$ & 2.821276 & -1.754387 & $\odot .337046$ \\
\hline 15 & 1 & $\odot$ & -0.533282 & 1.198070 & 2.227965 \\
\hline 16 & 1 & $\odot$ & -1.607740 & -1.270744 & 2.253049 \\
\hline 17 & 9 & $\odot$ & -1.292487 & -0.003964 & -1.752776 \\
\hline 18 & 1 & $\odot$ & 4.711199 & -0.715819 & $\odot .821068$ \\
\hline 19 & 1 & $\odot$ & 2.995634 & -2.785427 & $\odot .605129$ \\
\hline 20 & 1 & $\odot$ & 4.743320 & 1.659291 & -0.589184 \\
\hline 21 & 1 & 0 & 3.208187 & 2.521712 & -0.361105 \\
\hline 22 & 1 & $\Theta$ & 4.127003 & 1.979560 & 1.058760 \\
\hline 23 & $\overline{1}$ & $\Theta$ & 0.836864 & -3.077877 & -1.082140 \\
\hline 24 & $\overline{1}$ & 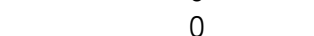 & 0.288265 & -2.777234 & 0.590789 \\
\hline 25 & 1 & $\odot$ & -0.347127 & -1.788972 & -0.748253 \\
\hline 26 & 1 & $\odot$ & -3.781188 & -2.266723 & 1.161415 \\
\hline 27 & 1 & $\odot$ & -4.105807 & -1.792705 & -0.511394 \\
\hline 28 & 1 & $\odot$ & -2.636496 & -2.706865 & -0.127872 \\
\hline 29 & 1 & 0 & -1.909410 & 3.338090 & -0.546788 \\
\hline 30 & 1 & $\odot$ & -1.375930 & 3.446257 & 1.137866 \\
\hline 31 & 1 & $\odot$ & -0.233500 & 2.939196 & -0.128832 \\
\hline-- & ----- & 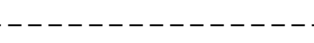 & 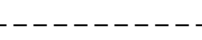 & 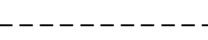 & - - - - - - - \\
\hline Don & $E($ RHF $)=$ & -1310.85250399 & A.U. a & 12 сус & \\
\hline & Convg & $0.9685 \mathrm{D}-08$ & & $-\mathrm{V} / \mathrm{T}=2 . \mathrm{C}$ & \\
\hline & $S * * 2$ & $\odot .000 \odot$ & & & \\
\hline
\end{tabular}


Variational C-PCM results

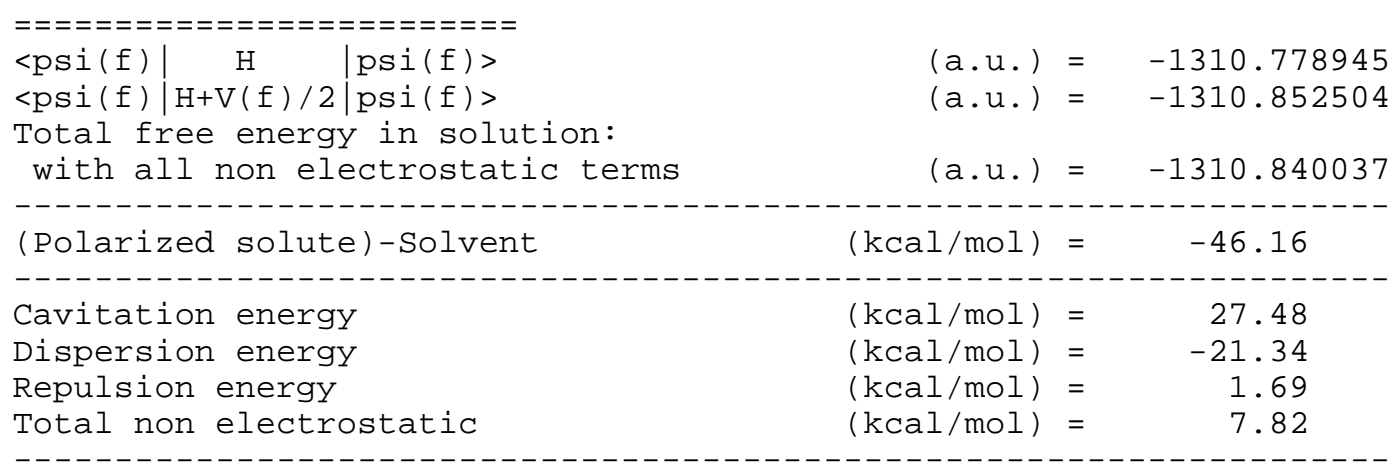

Spin components of $T(2)$ and $E(2)$ :
alpha-alpha T2 =
$\odot .1012834644 \mathrm{D}+00 \mathrm{E} 2=$
$-\odot .2857839224 D+0 \odot$
alpha-beta T2 =
$\odot .5621079851 \mathrm{D}+0 \odot \mathrm{E} 2=$
$-\odot .1656355417 D+01$
beta-beta T2 =
$\odot .1012834644 \mathrm{D}+00 \mathrm{E} 2=$
$-\odot .2857839224 D+\odot \odot$

ANorm $=\quad 0.1328410672 \mathrm{D}+01$

$\mathrm{E} 2=-0.2227923262 \mathrm{D}+01$ EUMP2 $=-\odot .13130804272535 \mathrm{D}+04$

\section{F-bridged 2-Fluoro-1,3-Dimethyl-1,3,2-diazaphospholene / 1,3-Dimethyl-1,3,2-}

diazaphospholenium Complex $4 \mathrm{~b}[\mathrm{~F}]\left(\mathrm{C}_{2}\right.$-Symmetric Transition State):

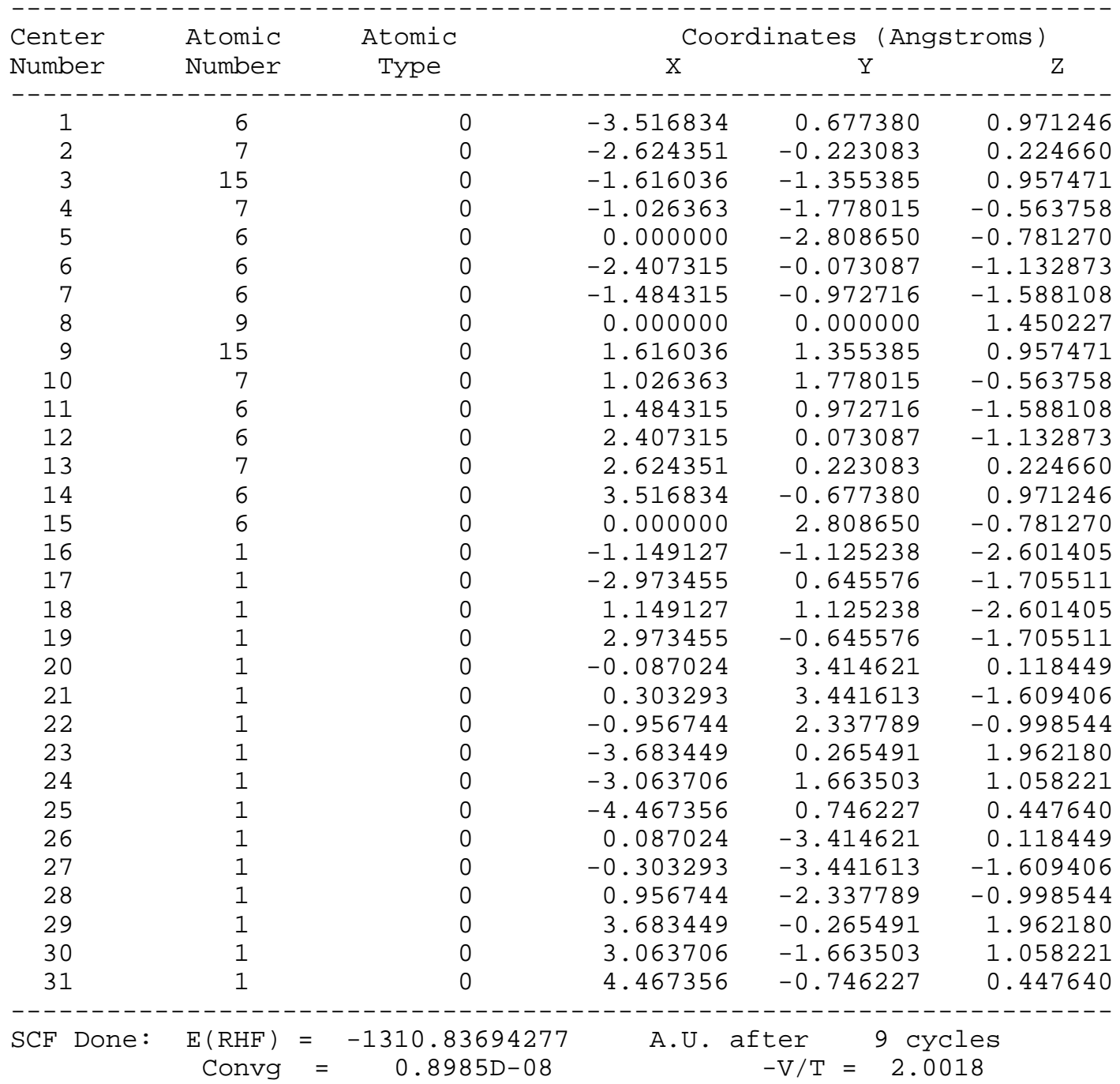


$\mathrm{S}^{* * 2}=0.0000$

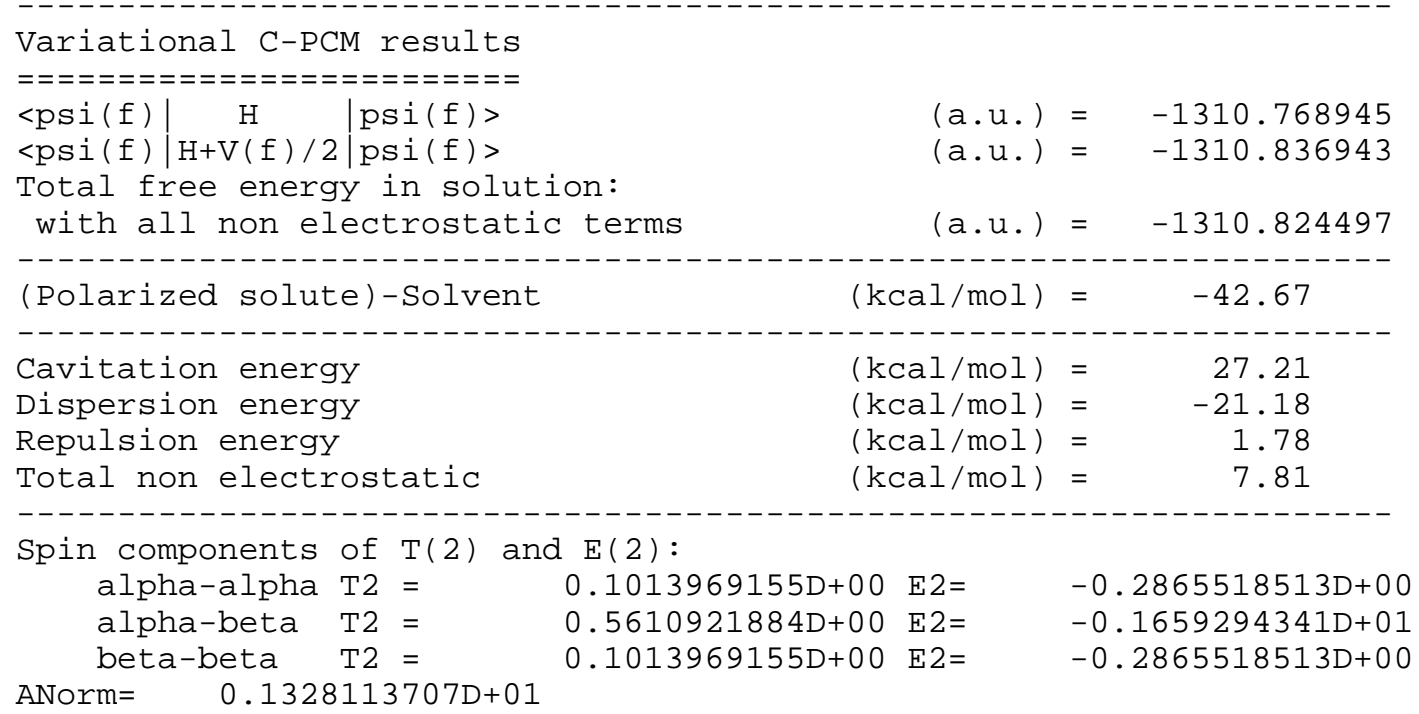

$\mathrm{E} 2=-0.2232398044 \mathrm{D}+01$ EUMP2 $=-0.13130693408161 \mathrm{D}+04$

Final atomic coordinates and energies for species in $\mathrm{CH}_{3} \mathrm{CN}$ solution at b3lyp/6-31+g** level:

2-Chloro-1,3-Dimethyl-1,3,2-diazaphospholene 1b[Cl]:

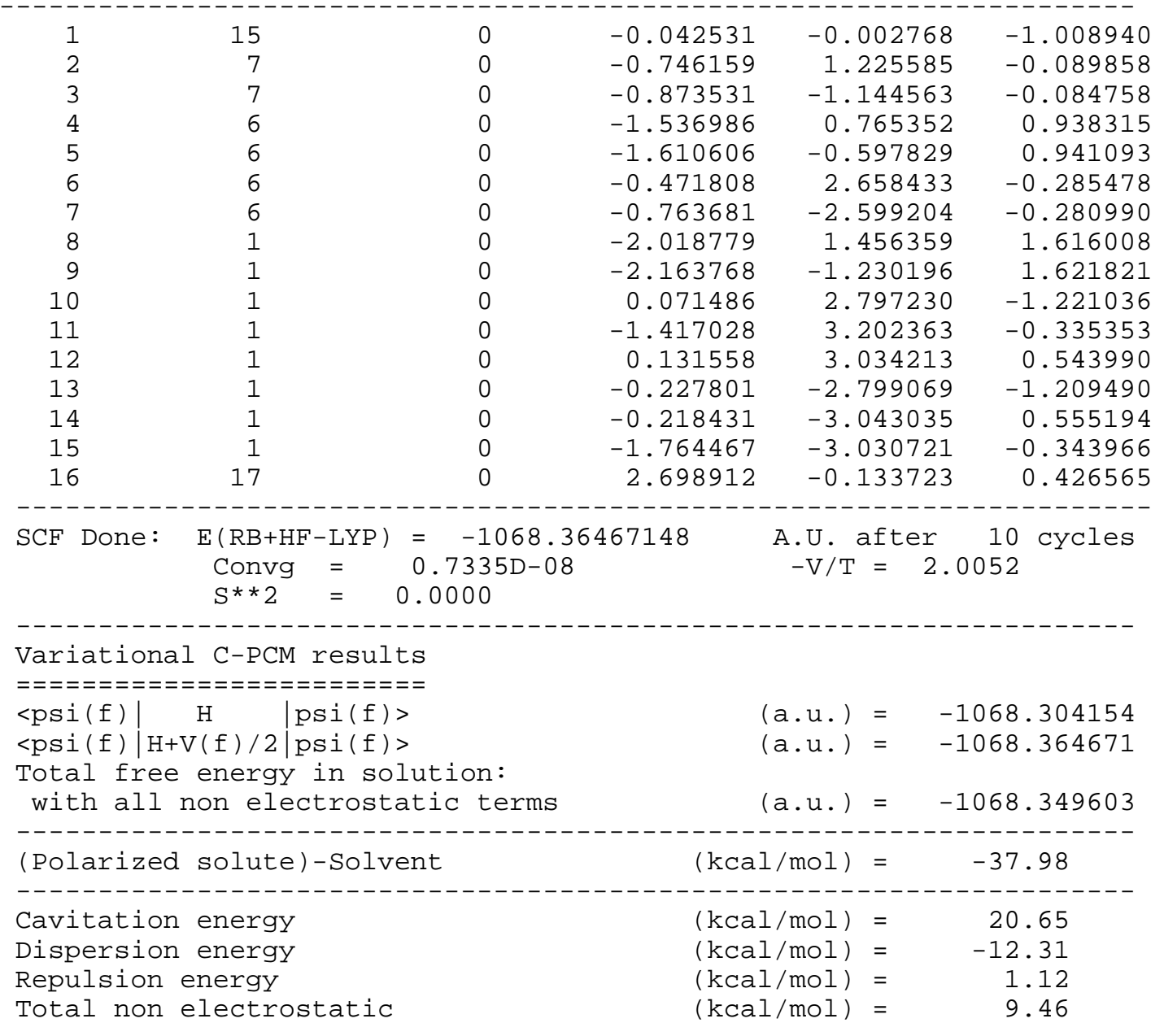


